University of South Florida

DIGITAL COMMONS

Digital Commons @ University of

@ UNIVERSITY OF SOUTH FLORIDA

South Florida

USF Tampa Graduate Theses and Dissertations

USF Graduate Theses and Dissertations

$11-13-2003$

\title{
Kinematic Analysis and Evaluation of Wheelchair Mounted Robotic Arms
}

Edward Jacob McCaffrey

University of South Florida

Follow this and additional works at: https://digitalcommons.usf.edu/etd

Part of the American Studies Commons

\section{Scholar Commons Citation}

McCaffrey, Edward Jacob, "Kinematic Analysis and Evaluation of Wheelchair Mounted Robotic Arms" (2003). USF Tampa Graduate Theses and Dissertations.

https://digitalcommons.usf.edu/etd/1428

This Thesis is brought to you for free and open access by the USF Graduate Theses and Dissertations at Digital Commons @ University of South Florida. It has been accepted for inclusion in USF Tampa Graduate Theses and Dissertations by an authorized administrator of Digital Commons @ University of South Florida. For more information, please contact digitalcommons@usf.edu. 
Kinematic Analysis and Evaluation of Wheelchair Mounted Robotic Arms

by

Edward Jacob McCaffrey

\begin{abstract}
A thesis submitted in partial fulfillment
of the requirements for the degree of Master of Science in Mechanical Engineering

Department of Mechanical Engineering

College of Engineering

University of South Florida
\end{abstract}

Major Professor: Rajiv Dubey, Ph.D.

Glen Besterfield, Ph.D.

Thomas Eason, Ph.D.

Date of Approval:

November 13, 2003

Keywords: manipulator, assistive, workstation, mobile, design

(C) Copyright 2003 , Edward Jacob McCaffrey 


\section{Table of Contents}

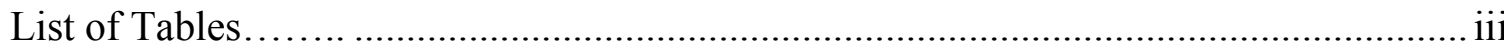

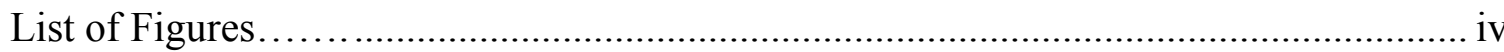

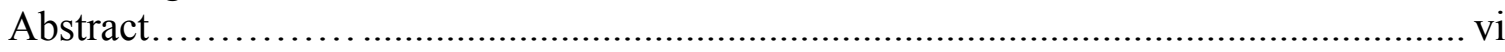

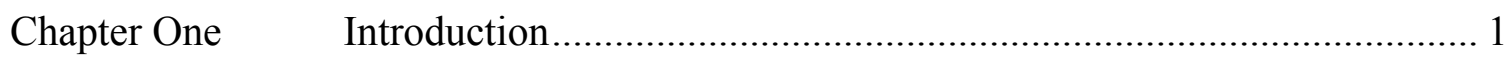

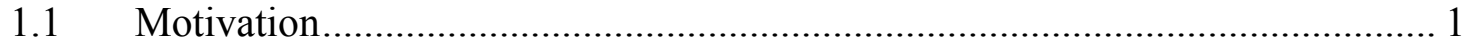

1.2 Objectives ............................................................................................ 2

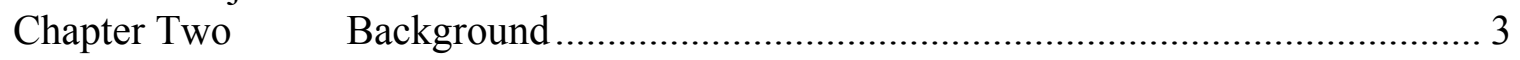

$2.1 \quad$ History of Rehabilitation Robotics ........................................................... 3

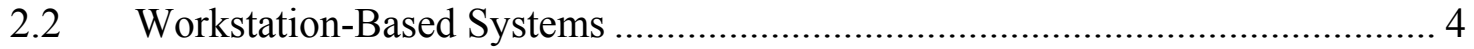

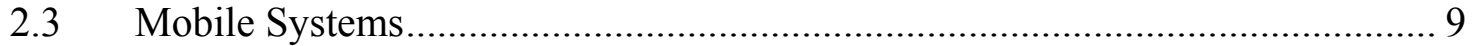

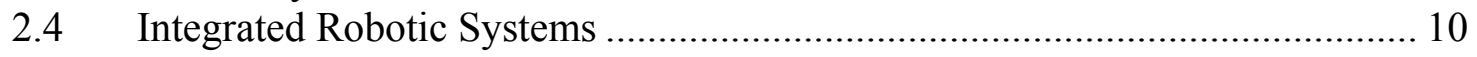

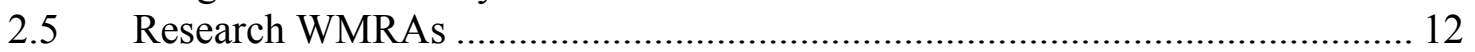

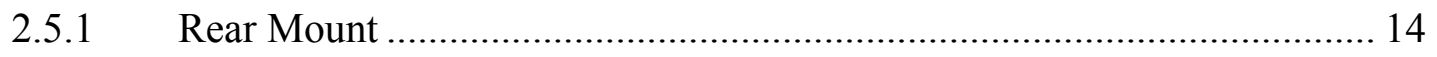

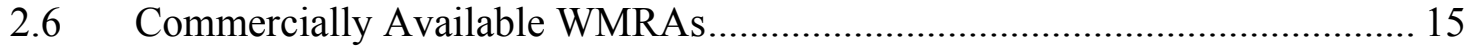

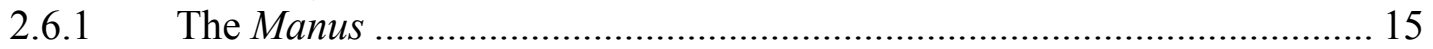

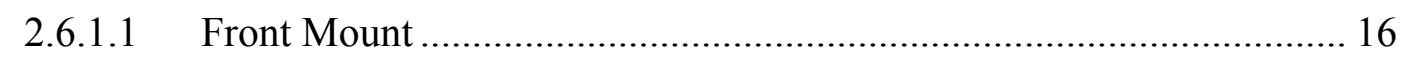

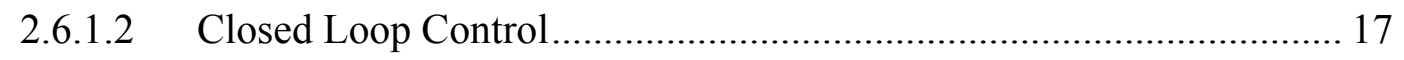

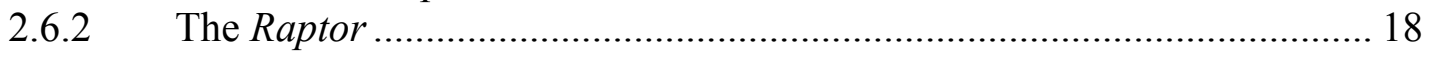

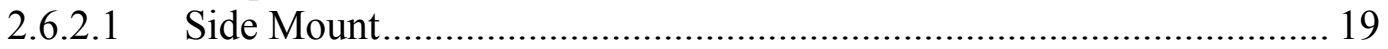

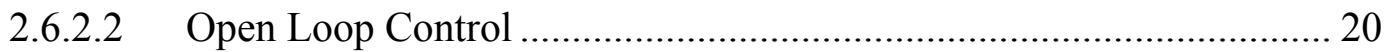

Chapter Three Procedure for Kinematic Analysis ................................................ 21

3.1 Determination of Workspace ................................................................ 21

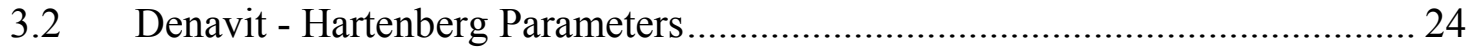

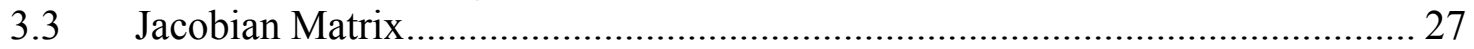

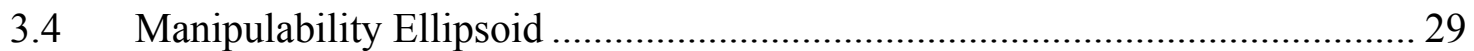

Inverse Kinematic Program .......................................................................... 32

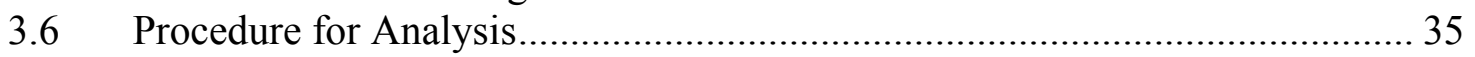

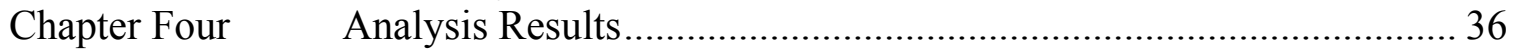

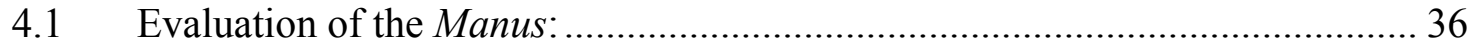

4.1.1 Vertical Planes ................................................................................. 42

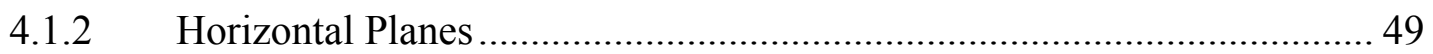

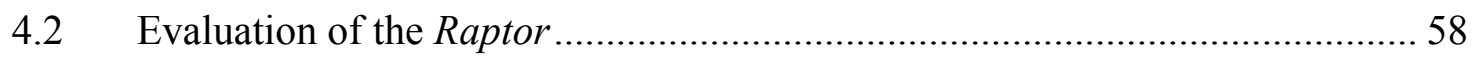

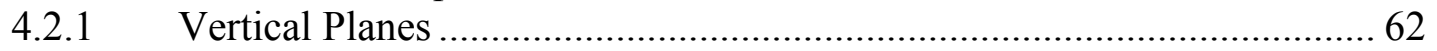

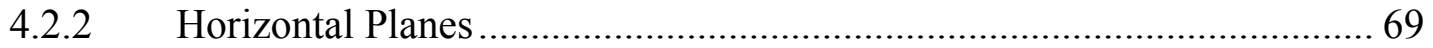

Chapter Five Design Insights and Recommendations ..................................... 79

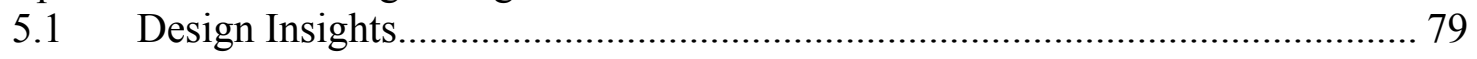


5.2 Design Recommendations ................................................................... 81

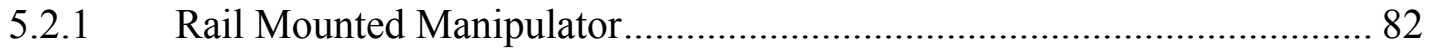

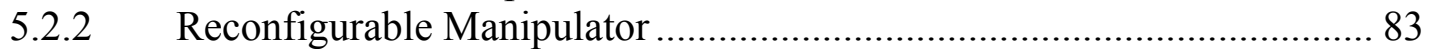

Chapter Six Summary and Future Work......................................................... 84

6.1 Design Recommendations and Insights ................................................. 84

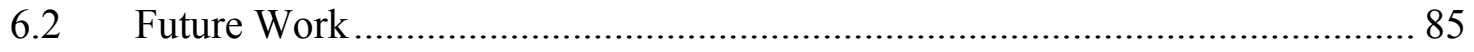

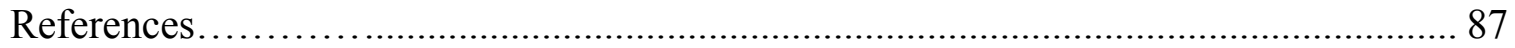

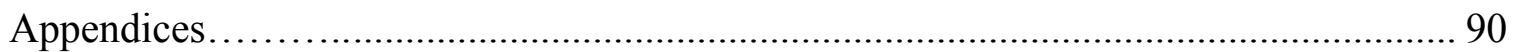

Appendix A: Manipulability Data ....................................................................... 91

Appendix B: Inverse Kinematic Program........................................................ 102 


\section{List of Tables}

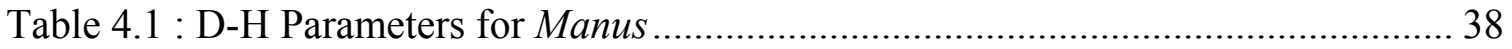

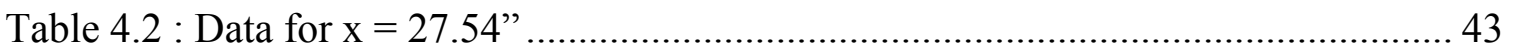

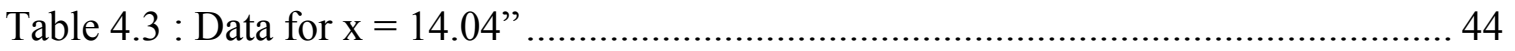

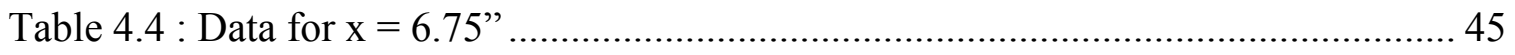

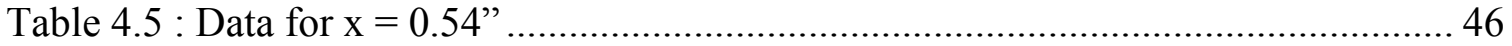

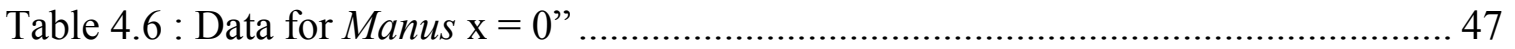

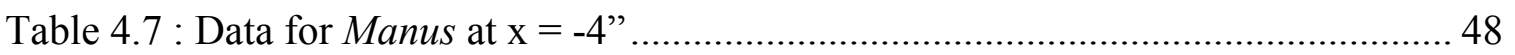

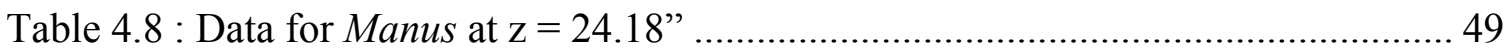

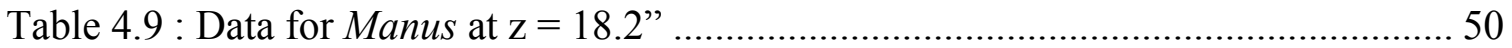

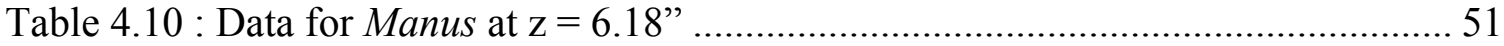

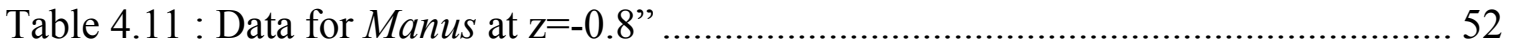

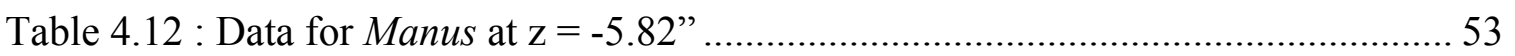

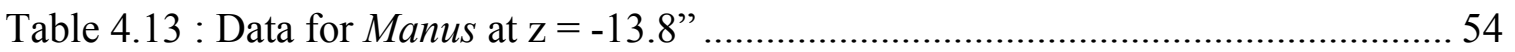

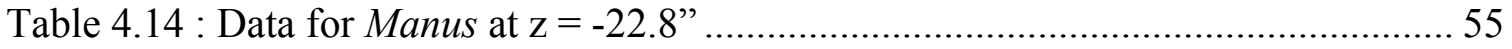

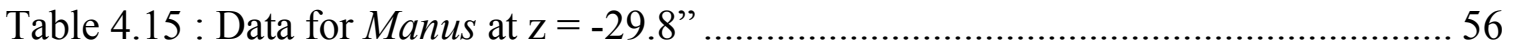

Table 4.16 : Qualitative Summary of Manus Effectiveness ......................................... 57

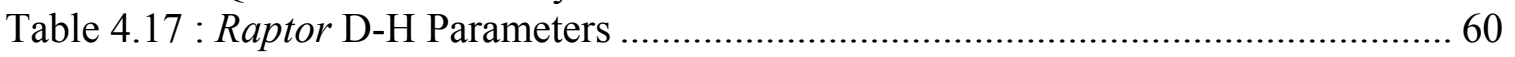

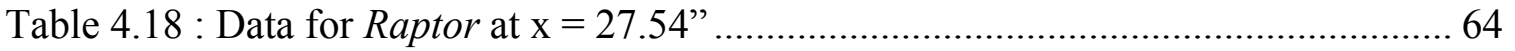

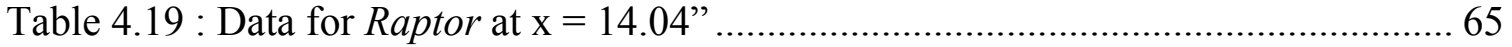

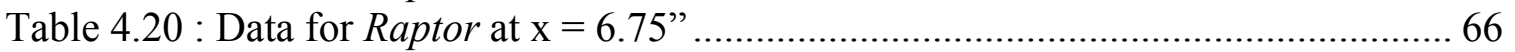

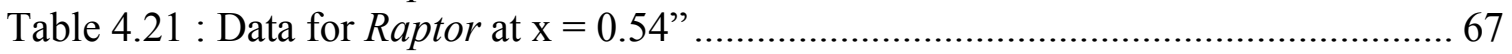

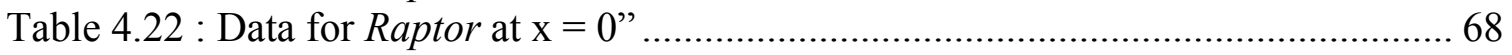

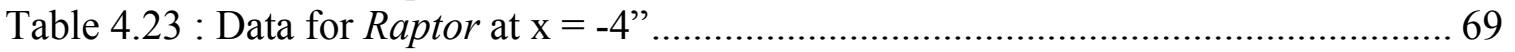

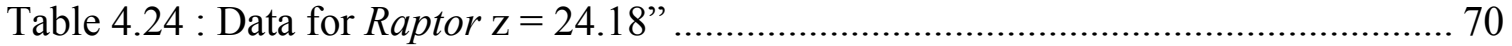

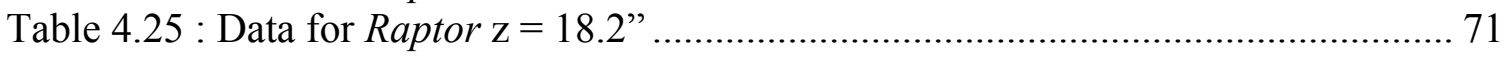

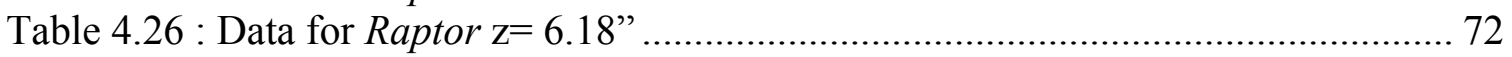

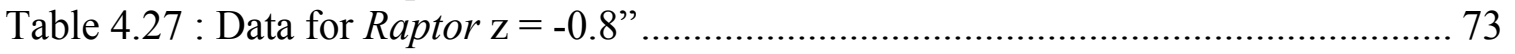

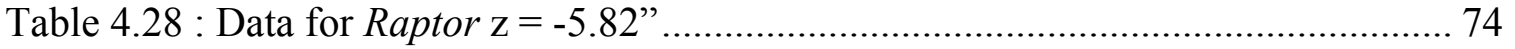

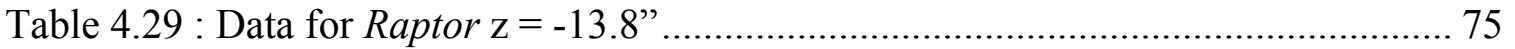

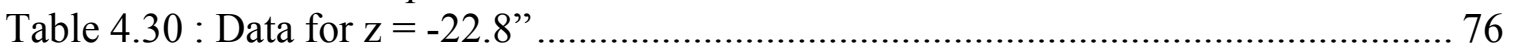

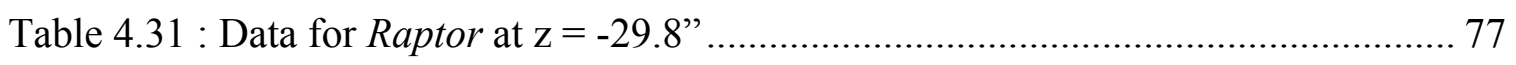

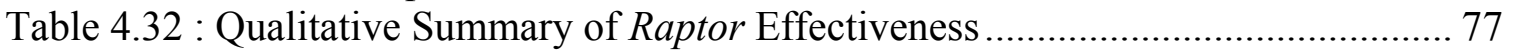




\section{List of Figures}

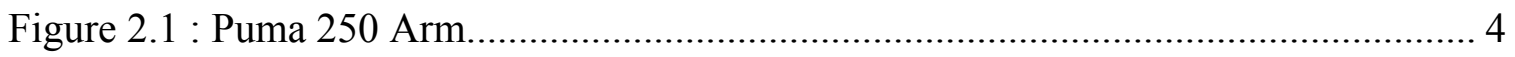

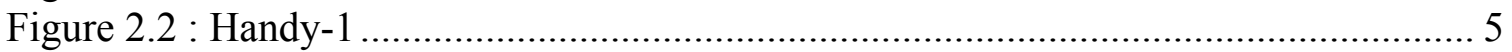

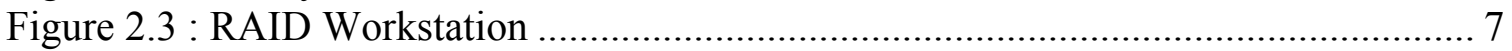

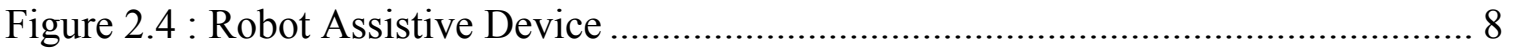

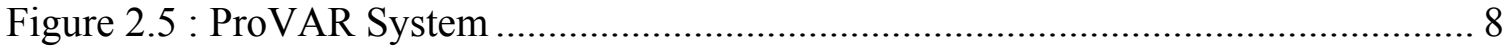

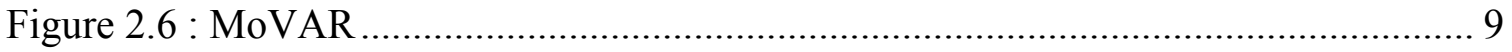

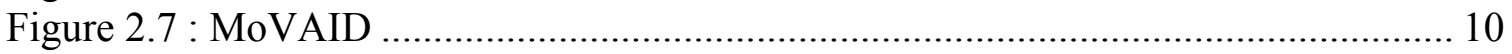

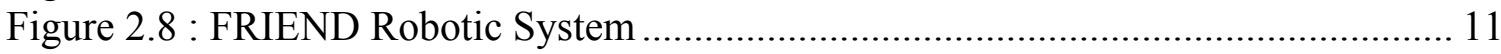

Figure 2.9 : TAURO Robotic System...................................................................... 12

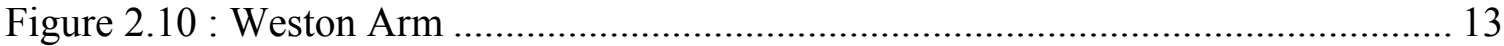

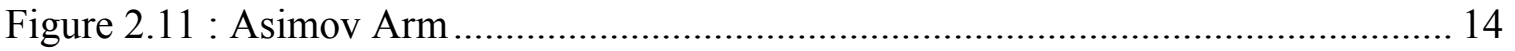

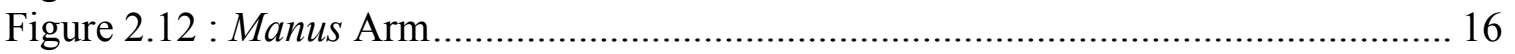

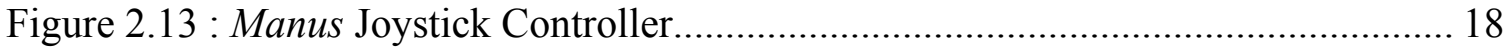

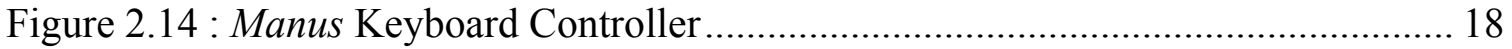

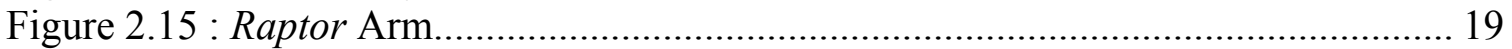

Figure 3.1 : Workspace Horizontal Planes ................................................................. 22

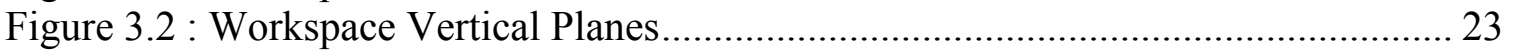

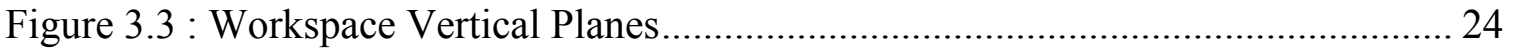

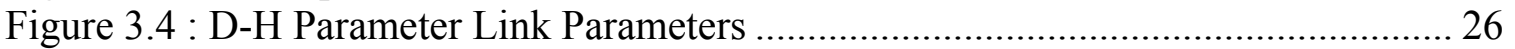

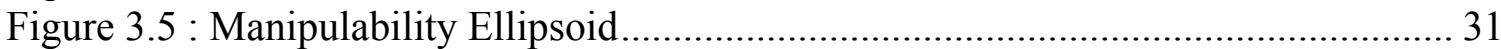

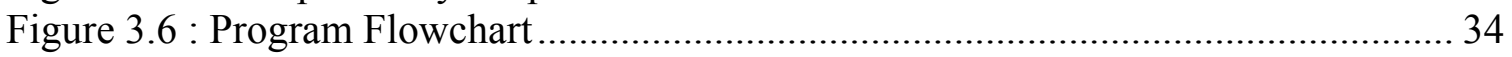

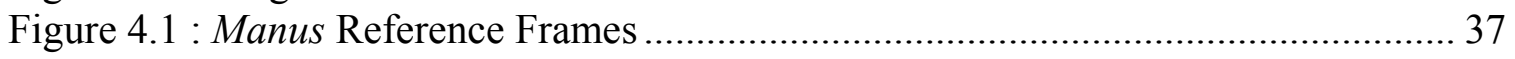

Figure 4.2 : Representation of the Manipulability Measure ......................................... 42

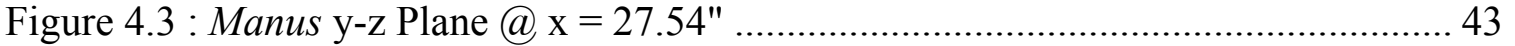

Figure 4.4 : Manus y-z Plane @ $\mathrm{x}=14.04 "$.................................................................... 44

Figure 4.5 : Manus y-z Plane @ $\mathrm{x}=6.75 "$....................................................................... 45

Figure 4.6 : Manus y-z Plane @ $\mathrm{x}=0.54 "$..................................................................... 46

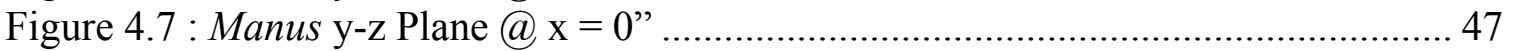

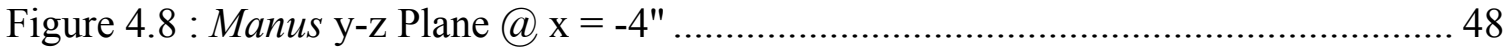

Figure 4.9 : Manus $\mathrm{x}-\mathrm{y}$ Plane $@ \mathrm{z}=24.18$ " .................................................................. 49

Figure 4.10 : Manus x-y Plane @ $\mathrm{z}=18.2$ " .................................................................... 50

Figure 4.11 : Manus x-y Plane @ $\mathrm{z}=6.18$ " .................................................................. 51

Figure 4.12 : Manus x-y Plane @ $\mathrm{z}=-0.8$ ".................................................................... 52

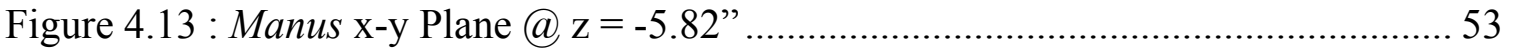

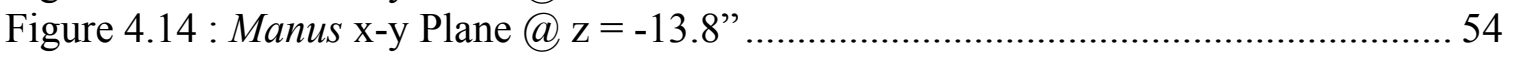

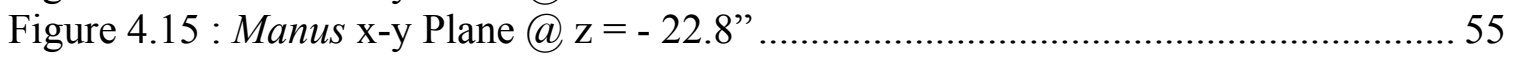




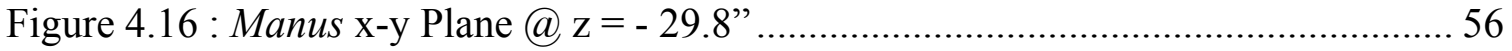

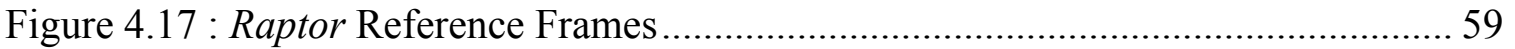

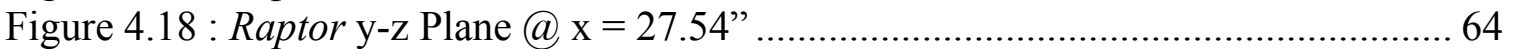

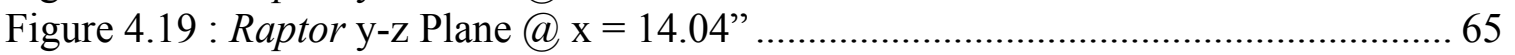

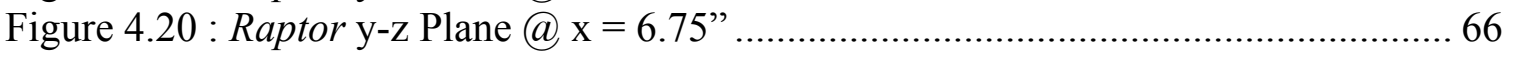

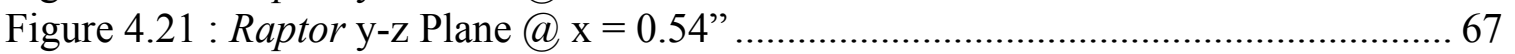

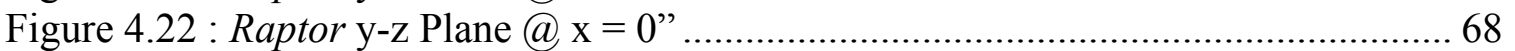

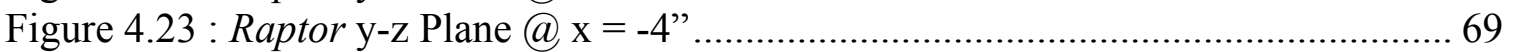

Figure 4.24 : Raptor $\mathrm{x}-\mathrm{y}$ Plane $@ \mathrm{z}=24.18$ " ................................................................. 70

Figure 4.25 : Raptor $\mathrm{x}-\mathrm{y}$ Plane $@ \mathrm{z}=18.2$ " ................................................................. 71

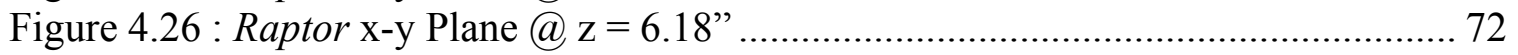

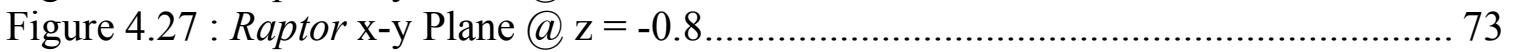

Figure 4.28 : Raptor $\mathrm{x}-\mathrm{y}$ Plane @ $\mathrm{z}=-5.82$ "............................................................... 74

Figure 4.29 : Raptor $\mathrm{x}-\mathrm{y}$ Plane $@ \mathrm{z}=-13.8$ "............................................................... 75

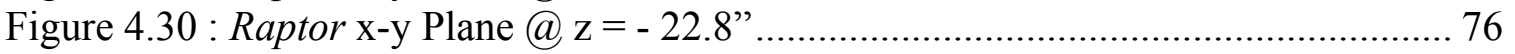

Figure 4.31 : Raptor $\mathrm{x}-\mathrm{y}$ Plane @ $\mathrm{z}=-29.8$ "............................................................... 77 


\title{
Kinematic Analysis and Evaluation of Wheelchair Mounted Robotic Arms
}

\author{
Edward Jacob McCaffrey
}

\begin{abstract}
The goal of this thesis is the kinematic analysis and evaluation of wheelchair mounted robotic arms. More specifically, to address the kinematics of the wheelchair mounted robotic arm (WMRA) with respect to its ability to reach positions commonly required by an assistive device in activities of daily living (ADL).

A robotic manipulator attached to a power wheelchair could enhance the manipulation functions of an individual with a disability. In this thesis, a procedure is developed for the kinematic analysis and evaluation of a wheelchair mounted robotic arm. In addition to developing the analytical procedure, the manipulator is evaluated, and design recommendations and insights are obtained.

At this time there exist both commercially-available and industrial wheelchair mountable robotic manipulators. The commercially-available manipulators (of which two will be addressed in this research) have been designed specifically for use in rehabilitation robotics. In contrast, industrial robotic manipulators are designed for speed, precision, and endurance. These traits are not required in assistive robots and can actually be dangerous to the operator if mounted onto a wheelchair. Manipulators to be used as WMRAs must be designed specifically for assistive functions in order to be utilized as a wheelchair mounted robotic arm.

In an effort to evaluate two commercial manipulators, the procedure for kinematic analysis is applied to each manipulator. Design recommendations with regard to each device are obtained. This method will benefit the researchers by providing a standardized
\end{abstract}


procedure for kinematic analysis of WMRAs that is capable of evaluating independent designs. 


\section{Chapter One}

\section{Introduction}

\subsection{Motivation}

A wheelchair mounted robotic arm can enhance the manipulability functions of people with disabilities. To better understand the effectiveness of a robotic arm, it must be analyzed with respect to its kinematics and the workspace in which it operates. Kinematics is defined as the relationship between the positions, velocities, and accelerations of the links of a robotic arm.

Data from the US Census Bureau Statistical Brief of 1993 showed that over 34 million Americans had difficulty performing functional activities ${ }^{1}$. Of this number, over 24 million were considered to have severe disabilities. Every year more and more people become disabled in a way which minimizes their use of upper extremities. These can be motor dysfunctions due to accidents, disease, or genetic predispositions.

The field of Rehabilitation Robotics has been created in an attempt to increase the quality of life and to assist in activities of daily living. Rehabilitation Robotics addresses assistive technologies as well as the traditional definition of rehabilitation: increasing or expanding the individual's mental, physical, or sensory capabilities. The primary focus of Rehabilitation Engineering ${ }^{2}$ and robotics is to increase the quality of life of individuals through increasing functional independence and decreasing the costs associated with the assistance required by the individual.

Robotic aids used in these applications vary from advanced limb orthosis to robotic arms. These devices can help in everyday activities for persons with severe physical disabilities limiting their ability to manipulate objects by reducing their dependency on caregivers. 
In the case of spinal injury or dysfunction, these aids are most appropriate for individuals with spinal deficiencies ranging from cervical spine vertebrae 3 through cervical spine vertebrae 5 . Below the cervical spine vertebrae 5 , individuals often can be served with simpler, more traditional assistive technology. Spinal fractures above cervical spine vertebrae 3 often require other medical necessities such as a respirator and daily attendants, thereby minimizing the need for assistive devices. Individuals with neuromuscular deficiencies such as muscular sclerosis can benefit from these robotic devices.

Individuals that require mobility assist devices such as a power wheelchair can benefit from various robotic devices because the power wheelchair provides a platform with which to mount the device as well as a power supply, using the wheelchair's batteries. There have been several attempts in the past to create commercially-viable wheelchair mounted robotic arms. Currently there are only two commercially available WMRAs available, the Manus (Exact Dynamics, Inc., Netherlands) and the Raptor (Applied Resources, Inc, NJ USA).

\subsection{Objectives}

The focus of this thesis is to analyze and evaluate WMRAs. To complete the analysis, an analytic procedure must be designed to systematically study the effectiveness of WMRAs. The procedure is executed and the manipulator is then evaluated using criteria specific to rehabilitation applications. A completed evaluation can provide design recommendations and possibly insights into design modifications or new manipulator geometries which better fulfill the specific needs of a rehabilitation robotic manipulator.

The objectives of this thesis are the following:

- Create a procedure for quantitative kinematic analysis

- Evaluate the Manus and Raptor arm using this procedure

- Obtain design recommendations and insights based on the evaluation 


\section{Chapter Two}

\section{Background}

\subsection{History of Rehabilitation Robotics}

There have been various attempts over the years to create robotic assistants for individuals with various levels of disabilities. For over 30 years, research has progressed in the field with only partial commercial success. An early attempt at telemanipulators was done at the Case Institute of Technology during the early 1960's. The Case system ${ }^{3}$ was a floor-mounted, powered exoskeleton. It was controlled by an operator who wore a head-mounted light source which triggered light sensors in the environment. By looking at specific points in the room, the operator could trigger the light sensors and initiate one of several preprogrammed gestures which were stored on magnetic tape. A later development allowed for Cartesian movement and direct control of individual joints along with myoelectric signals for velocity control.

One of the first attempts at rehabilitation robotics included the Rancho "Golden" arm ${ }^{4}$ designed in 1969 at Rancho Los Amigos Hospital in Downy, California. The arm was an electrically-driven 6 Degree Of Freedom (DOF) robotic arm which mounted to a powered wheelchair and was controlled at the joint level by an array of tongue-operated switches. Further discussions on the topic of the controllability of the arm commented on both successes and failures of the design. The successes with the project can be attributed to the important role that proprioceptive feedback plays in the control of a persons own extremities $^{5}$. These pioneering research projects provided a framework for future development.

Assistive robotics can be grouped into one of three categories:

- Workstation robots which operate in stationary, well-structured environments

- Mobile assistive robots which travel about the room and have a manipulator arm 
- Wheelchair mounted robotic arms which mount a manipulator arm onto the individual's wheelchair to provide assistance throughout the day

\subsection{Workstation-Based Systems}

The very first rehabilitation robotics applications focused on using commerciallyavailable industrial manipulators and modifying them for rehabilitation applications. An example of these manipulators is the PUMA 250 shown in Figure 2.1. A factor which limits the use of industrial robotic arms in rehabilitation is the basic difference in operational requirements. Industrial arms are designed to work at high speed in an environment where there are no humans. This reason alone would limit their use for reasons of safety of the operator. For applications in a human-intensive workspace, assistive robotic arms need to be mechanically limited to low velocity and accelerations.

A more modern version of this workstation approach is the RAID (Robotic Assistance in Daily Living) system which will be discussed in more detail later.

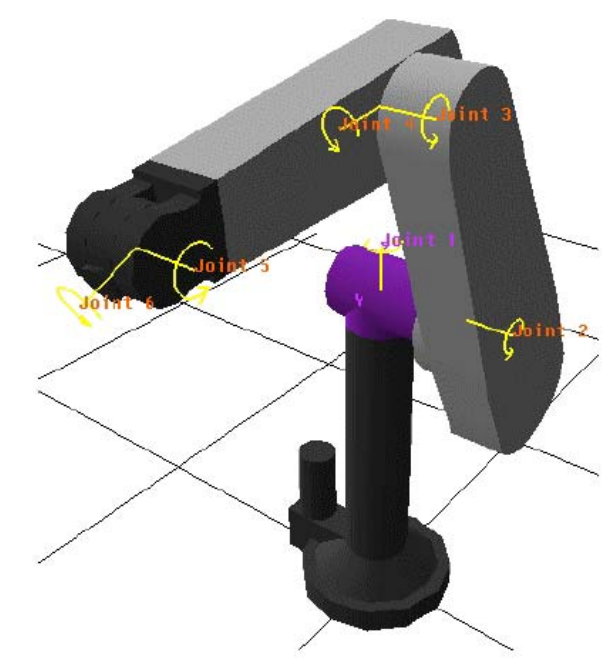

Figure 2.1 : Puma 250 Arm

The Robotic Aid Project ${ }^{6}$ was an attempt to create a system for users with quadriplegia. The project was an integration of a PUMA 250 industrial manipulator arm, microprocessor, multi-line monochrome display and speech synthesis and recognition systems. Limitations with the speech-recognition systems and computational power of 
the day restricted the success of the program. The processing ability of the contemporary computers did not allow for real-time reverse kinematics of the arm. This limited the arm to merely replaying preprogrammed actions. Individual joints of the arm could be manipulated but coordinated, real-time multi-joint maneuvers were impossible.

As more application-specific robotic arms and computers with increased computational power became available, arms with controllers could now be mounted onto mobile platforms. At first these systems were simply rolling bases which then increased in complexity and degrees of freedom to include powered mobile robots.

Handy $-1^{7}$ is a robotic arm mounted to a non-powered wheeled base to assist in very specific activities of daily living (ADL). Handy-1 was developed in 1988 to provide persons with severe disabilities assistance at mealtimes. Since its initial introduction the unit has expanded capabilities and is now capable of providing assistance in a broader number of activities of daily living (ADL). Handy-1 is capable of assisting individuals with personal hygiene, eating and drinking, and the application of make-up. During user trials, women specifically asked if the unit would be capable of applying cosmetic products. Shortly after the trial, the design was upgraded with a new tray and gripper accessory. Each ADL task has a specific tray to accomplish its goal. Handy-1 is shown in Figure 2.2 and is based on a 5 DOF lightly modified industrial manipulator.

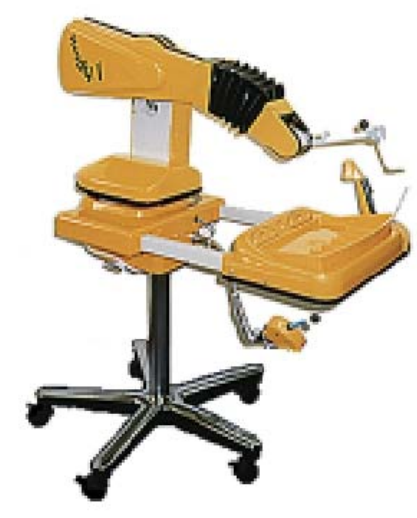

Figure 2.2 : Handy-1

In the feeding mode the operator controls the robot through an interface that uses lights which move across the available food trays, and a button that selects the item 
desired. Once the button is pressed, the robot scoops up the selected food and brings it to a predetermined place near the operator's mouth. Once the user has consumed the food, the operator presses the button again and the robot returns to the food selection mode. This process is repeated until the operator is finished.

This assistive device does not eliminate the need for a personal assistant but allows for individuals to have an increased level of self-sufficiency. In user trials almost invariably all the users believed the device to significantly increase their quality of life. An advancement of the technology of Handy-1 is being explored with the Robotic Aid to Independent Living ${ }^{8}$ (RAIL) project. RAIL improves upon the Handy-1 design by incorporating a new controller for better manipulator control, a 3D simulation tool for modeling virtual scenarios and attachment of sensors to assist set up and position error determination.

The Wessex robot ${ }^{9}$ (Bath Institute of Medical Engineering) is a trolley-mounted mobile robot of modified SCARA geometry. A SCARA arm has two revolute joints in the horizontal plane, allowing it to reach any point within a horizontal planar workspace defined by two concentric circles. In modified SCARA configuration, most of the joints operate in the horizontal plane. All vertical movement is achieved through the use of a single vertical actuator.

The Wessex robot suffered from several design shortcomings. One example was its limited manipulator reach. The manipulator was designed to grasp only items on a tabletop. Because of this limitation, it was unable to pickup items off the ground. The trolley was not powered and was pushed into location by the daily assistant.

In user trials the operator felt limited by the number of programs which it could store and that the trolley was not powered. The user felt that if the trolley were able to be driven by remote control it could be used to retrieve or manipulate objects within the same room. This could allow the user to adjust the thermostat or retrieve a drink from an attached kitchen.

The RAID workstation ${ }^{10}$ shown in Figure 2.3 was designed to be a workstation assistive robot system. It is comprised of a 6 DOF robotic arm mounted onto a linear 
track in a well-controlled environment. In the figure the manipulator can be seen near the top of the shelf in the center of the cabinet.

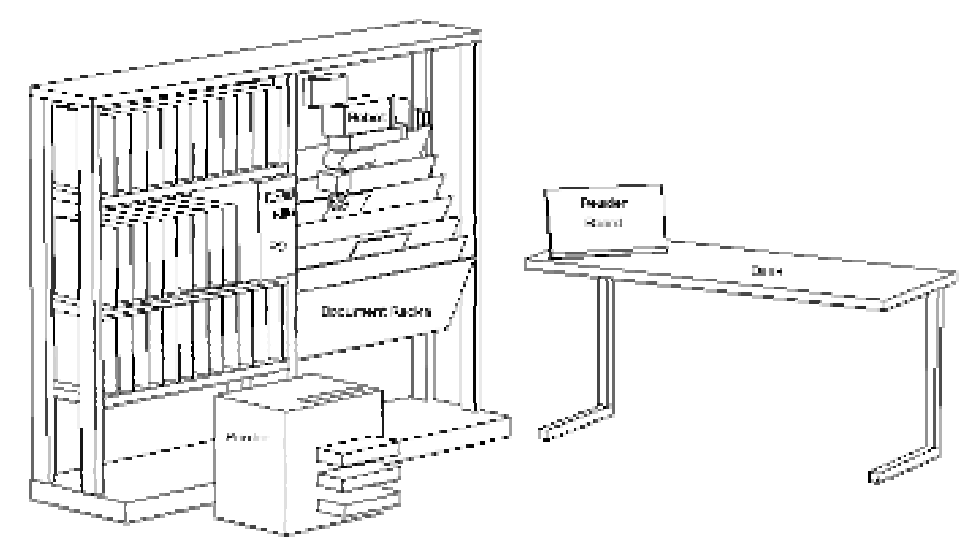

Figure 2.3 : RAID Workstation

The RAID system provides benefits that are enhanced by the formal structure provided by a workstation environment. This organization allows the manipulator arm to repeatedly move and acquire items needed by the operator using preprogrammed functions and routines. At this time the RAID system is currently under evaluation in Europe.

The Robotic Assistive Device ${ }^{11}$, shown in Figure 2.4, is a robotic arm currently under development by the Neil Squire Foundation in Vancouver, Canada. The RAD is a 6 DOF workspace mountable manipulator that uses a serial port computer interface. The manipulator is controlled through a graphical user interface (GUI) utilizing icons to symbolize predefined tasks. The system consist of several modules which when combined create an arm with a cylindrical reach of approximately 55" and a height of 110 ". The arm can be mounted on various surfaces and has good repeatability at 0.12 " and relatively large payload capacity of $9.5 \mathrm{lbs}$. Most rehabilitation specific manipulators have maximum payloads of 5 pounds or less. 


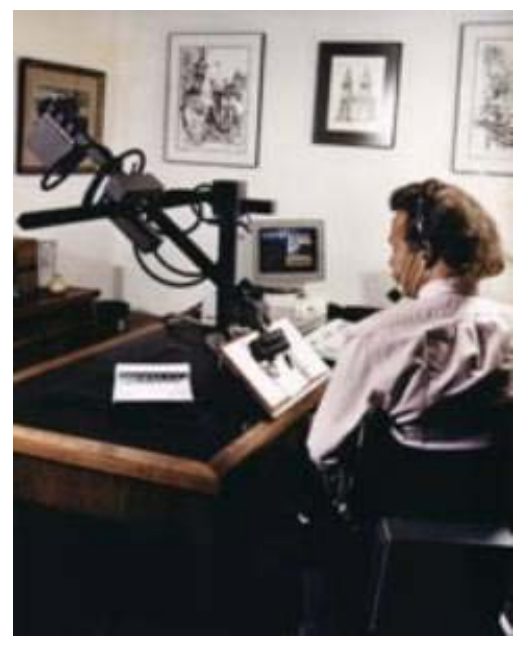

Figure 2.4 : Robot Assistive Device

The ProVAR ${ }^{12}$ (Stanford, CA) is a system based on a Puma 260 robotic arm designed to operate in a vocational environment. The ProVAR manipulator shown in Figure 2.5 is the next generation of the DeVAR system and expands upon the previous research by reducing operating costs and increasing overall usefulness.

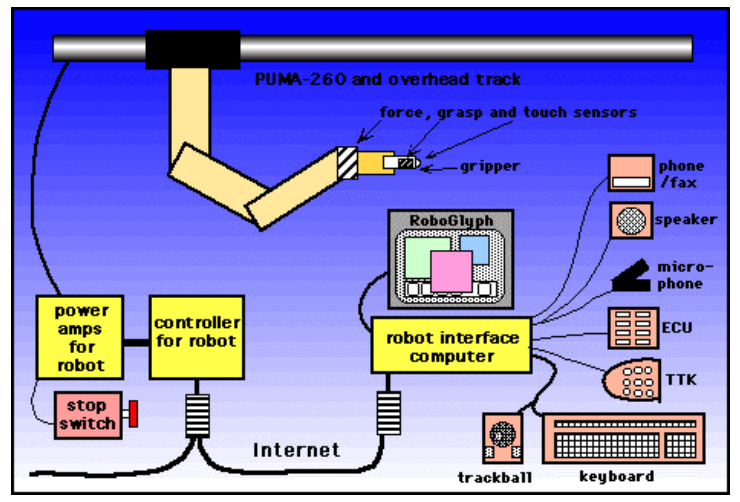

Figure 2.5 : ProVAR System

The ProVAR system uses a web-based virtual environment to model the functionality of the manipulator. In this way the operator can examine potential arm movements for a given task, and if the simulation is successful, the action can be 
initiated. In this way, the actions of the arm and its interactions within its workspace can be seen before any action is taken. The primary goals for ProVAR are more functionality per dollar, easier operator control, and higher system reliability compared with the previous generation of vocational assistive robots.

\subsection{Mobile Systems}

Mobile systems are capable of assisting individuals with disabilities. These systems include a mobile base, various sensors and a manipulator arm. An early version of one such system is the Mobile Vocational Assistant Robot $^{13}$ (MoVAR). MoVAR, shown in Figure 2.6, utilizes an omni-directional mobile platform mounting a PUMA-250 robotic arm as well as several sensors including a remote viewing camera, force and gripper proximity sensors.

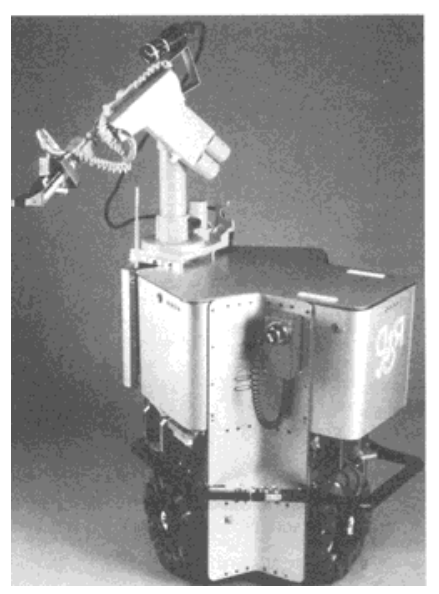

Figure 2.6 : MoVAR

MoVAID is an advanced version of the MoVAR system, designed specifically for home use. MoVAID improves upon the previous model by applying the lessons learned in laboratory testing to assist in common tasks around the home such as cleaning and food preparation. MoVAID incorporates a variety of sensing devices both mounted to the manipulator and the base. In Figure 2.7 MoVAID can be seen along with the various 
sensors that are located on the manipulator arm. Sensors mounted to the first link of the arm include a pair of cameras used for stereo vision and a laser localization system used in task execution.

The MoVAID system uses active beacons positioned within the room that provide reference data to determine its location and orientation. In addition to position detection, the unit also has ultrasonic range detectors and an active bumper that disables the device should an impact occur.

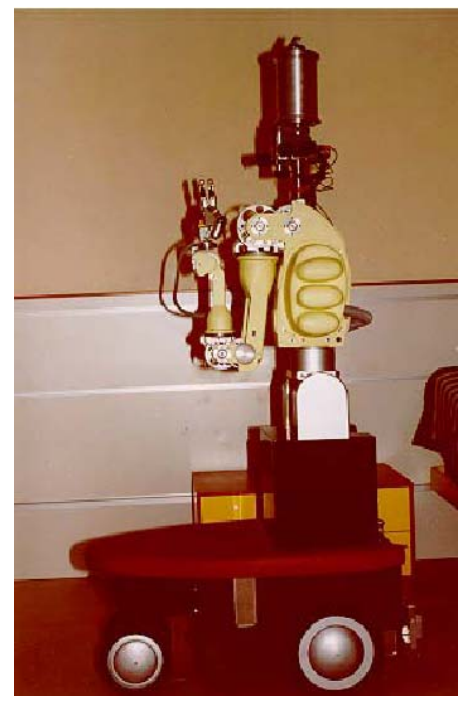

Figure 2.7 : MoVAID

The robotic arm used by MoVAID has 8 DOF and a three-fingered gripper with two degrees of freedom. The gripper was originally designed as a prosthetic device specifically to have excellent dexterity. The increased agility provided by the gripper over more traditional end-effectors allows MoVAID to be very effective in the unstructured home environment.

\subsection{Integrated Robotic Systems}

Research is being conducted on robotic assistive devices with increased autonomy and some artificial intelligence. This increased integration of robotic arms and other 
sensors has led to some increasingly capable designs. Although still in development, these designs offer even greater potential as assistive devices for the future.

The FRIEND ${ }^{14}$ robotic system is a Manus arm mounted onto a wheelchair and integrated with stereo vision, dedicated computer control, and specialized software. Besides programming with a keypad or joystick, the FRIEND system, shown in Figure 2.8 , is capable of being programmed via a haptic interface glove. The haptic glove allows the operator / programmer to feel what the robot feels through feedback to the user. A Haptic glove is put on and the action, such as pouring a glass, is completed and stored into the computer for future use. The action can then be replayed at a later time as a predefined user function. The operator may also control the arm through verbal commands using an integrated voice recognition system.

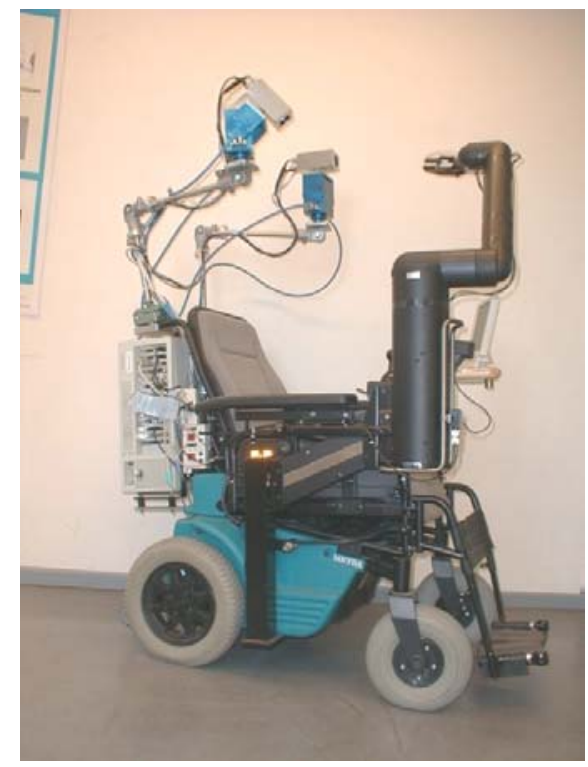

Figure 2.8 : FRIEND Robotic System

Another design is the TAURO. The TAURO is an integrated robotic system using off-the-shelf components such as a power wheelchair, Manus manipulator, ultrasonic sensors, camera and computers. TAURO is a mobile service robot being developed for inspection, stocktaking and documentation tasks in indoor environments. The TAURO system integrates the movement of the wheelchair and the operation of the manipulator. 
In this way if the goal is out of reach of the manipulator, the wheelchair will move on a path toward the goal until the manipulator can reach its goal. This coordinated control is a significant advance in the use of WMRAs. Although not specifically designed for rehabilitation robotics tasks, it would be readily adaptable to the task. The TAURO system can be seen in Figure 2.9.

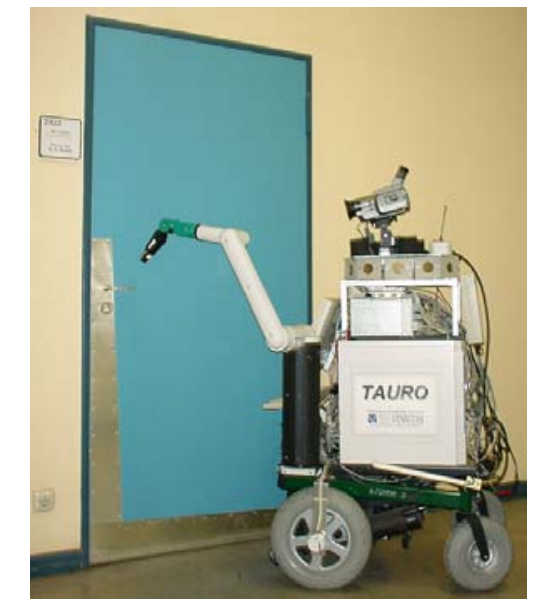

Figure 2.9 : TAURO Robotic System

\subsection{Research WMRAs}

Wheelchair mounted robotic arms (WMRAs)

Combining the idea of a workstation and a mobile robot, a WMRA mounts a manipulator arm onto a power wheelchair. In the past, industrial manipulators have been too large and heavy to be mounted onto a power wheelchair. An industrial manipulator mounted onto the wheelchair would have excessively hindered the operator's ability to maneuver the chair through doors and hallways. More recently, manipulator arms have been specifically designed to be used as WMRAs.

Currently there are two production wheelchair mounted robotic arms (WMRAs) : the Manus, manufactured by Exact Dynamics, and the Raptor, manufactured by Applied Resources. Some WMRAs under development are the Helping Hand (USA), Weston Arm (UK), and the Asimov (Sweden). 
Helping Hand system ${ }^{15}$ (Kinetic Rehabilitation Instruments, Hanover Massachusetts) is a 5DOF robotic arm being developed for commercial use. Its design is modular in nature and can be mounted to the side of a power wheelchair. The Helping Hand operates by joint control and is manipulated by using switches to control individual joints.

The Weston robotic arm (Bath Institute of Medical Engineering) utilizes a vertical actuator mounted to a wheelchair with the main rotary joints (shoulder, elbow, and wrist) constrained to move in the horizontal plane. This is the continuation of the trolley mounted Wessex robot arm research.

The Weston robotic system shown in Figure 2.10 is still under development. The Wessex arm is larger than both the Manus and the Raptor designs due to the use of a prismatic first joint. A prismatic joint moves in a linear sliding motion along a track. The other joints of the arm utilize a modified SCARA design as described in the Wessex manipulator.

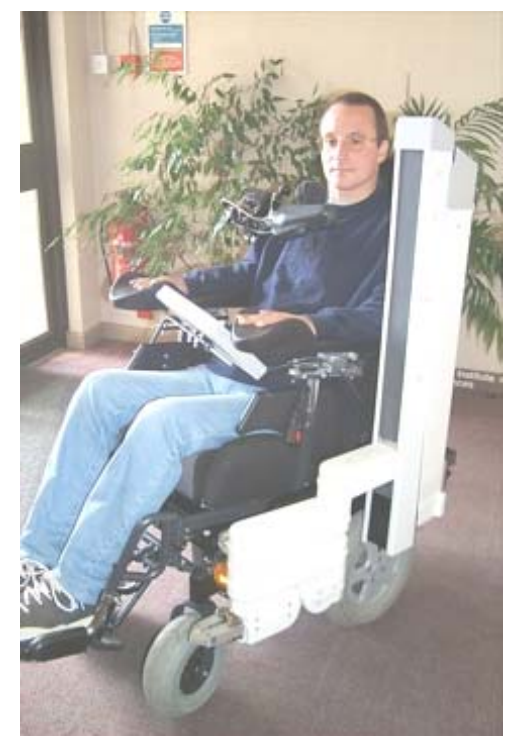

Figure 2.10 : Weston Arm 
Another arm currently under development is the Asimov ${ }^{16}$. The Asimov is a modular manipulator design with the motors and controls distributed throughout the arm. A computer rendering of the Asimov is shown in Figure 2.11. The modularity of the design allows for multiple mounting locations on a wheelchair or stationary application with various workspace geometries.

The concept of a modular manipulator has several benefits. This provides the opportunity for one manipulator that can be used in either a mobile or workstation environment. Different link geometries can be explored to create the optimum design for any given application. Asimov models have been shown with all three possible mounting positions: front, side and rear. Without physical models to test the efficacy of the design, it is unknown how well the design would integrate into real-world applications.

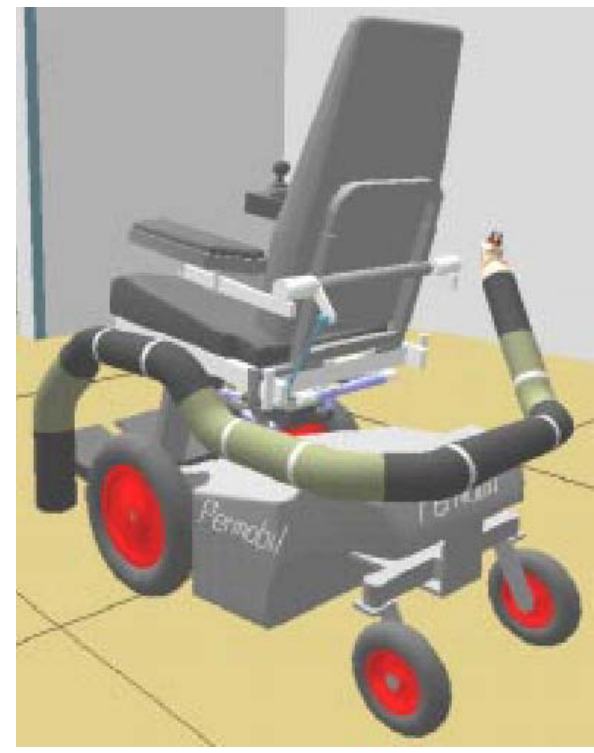

Figure 2.11 : Asimov Arm

\subsubsection{Rear Mount}

Several design considerations must be met before deciding on where, on a power wheelchair, to mount a robotic arm. The foremost design consideration is the safety of the operator $^{17}$. The mount must be sturdy and rigid and not compromise the structural integrity or the functionality of the chair in any way. Next the robotic arm must be 
mounted in such a way that it has a minimum footprint outside the footprint of the chair itself.

One of the potential benefits of a rear-mounted arm is that it will not increase the width of the wheelchair when not in use. Assuming that the arm is capable of being stowed behind the wheelchair, the arm would not create a distraction for individuals interacting with the person. Additionally, a rear-mounted arm would not be a physical obstruction during transfer into and out of the wheelchair.

Rear-mounted robotic arms have drawbacks caused specifically by the mounting location. In order for a manipulator to reach to the front of the wheelchair the manipulator must have longer link lengths than a front- or side-mounted design. The longer link lengths required by the dorsal (rear) mount require greater torque from the motors and increased loads on the bearings. At this time there are no commercially available WMRAs that are mounted to the rear of the wheelchair. It should be noted that there is an optional rear mounting bracket available for the Raptor but this eliminates most of the ability of the arm to reach directly in front of the chair.

\subsection{Commercially Available WMRAs}

\subsubsection{The Manus}

The Manus manipulator arm is a fully deterministic manipulator. A fully deterministic arm can be programmed in a manner comparable to industrial robotic manipulators. At any time the joint angles are known by the controller and the exact gripper position is known. The Manus has been under development since the mid 1980's and entered into production in the early 1990's. A picture of the Manus mounted onto a Permobil Max90 wheelchair is shown in Figure 2.12. 


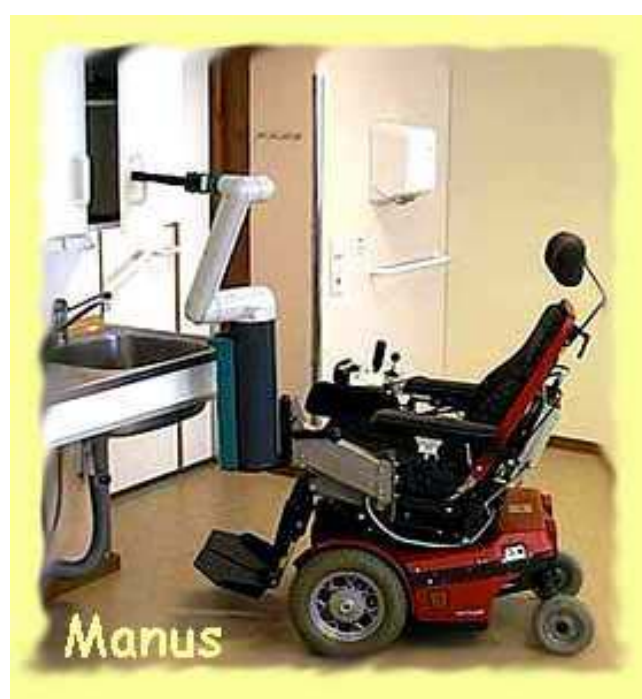

Figure 2.12 : Manus Arm

\subsubsection{Front Mount}

There are several possible mounting locations for a WMRA ${ }^{18}$. The mount may be in the front, side or rear of the wheelchair. The Manus utilizes a front mounting location to the left of the operator's left knee. The first joint of the arm rotates about the z-axis (floor to ceiling) and is located approximately two inches above the level of the arm rest of the power wheelchair. This location allows for good manipulation of objects that are above the plane of the wheelchair seat, and most importantly the operator's face and lap.

The front mount offers greater access to the operator's immediate working environment. The lap, tray top on armrests, and the mouth location can all be considered the immediate environment of the operator. Manipulation of objects in these areas is optimized with this mounting location. The high mounting position near the knee allows for good access to high objects such as items on shelves or operating doors on high cabinets. Objects in front of the chair are also readily manipulated.

Additionally, the front mounting of the robotic arm provides excellent accessibility to high shelves and allows the execution of various activities of daily living.

The front mount for a WMRA has limitations. Users have commented the front mounting makes the manipulator arm obtrusive and can create uncomfortable social 
tensions with people unfamiliar with robotic technology. This was noted as a hindrance in long-term Manus trials ${ }^{19}$. The mounting location also limited the ability of the operator to put their legs under desks, tables, and sinks in clinical evaluations. ${ }^{19}$

\subsubsection{Closed Loop Control}

The Manus manipulator is controlled by a joystick and a keypad. The joystick is used to manually operate the manipulator is shown in Figure 2.13. Manus can also perform coordinated control of multiple joints with preprogrammed gestures using the 16- button keypad shown in Figure 2.14. Gestures can be taught to the Manus and stored for future use via the keypad. With the use of the two input devices, the operator can run pre-programmed routines or directly control the manipulator in real time.

The controller converts the inputs from an input device into a signal which directly controls the robotic arm. There may be a direct or indirect link between the input device and the output signal. This control may be a simple proportional control or a more complex method where input position is converted into arm velocity output.

Closed loop systems are commonly used in industrial robotics. These systems permit accurate repeated motions of robotic manipulators to accomplish specific tasks within a manufacturing cell. A manufacturing cell is a highly structured environment which permits high productivity by eliminating positioning variances.

Rehabilitation workstations are very similar to the workspace originally used by industrial robotic arms. A closed loop system is useful in rehabilitation robotics applications by allowing pre-programmed actions and maneuvers. Pre-programmed gestures can be as simple or as complicated as required to accomplish a specific task. Closed loop control also allows further integration of the arm into more complicated and intelligent systems which can assist the operator. The MANUS system is a version of a closed loop system.

The downside to closed loop systems is their higher initial cost. The drive motors for the links must have encoders or some other form of feedback to send to the controller. Often the increased productivity, programmability, and system interoperability of a 
closed loop system can compensate for this increased initial cost by offering more "bang for the buck".

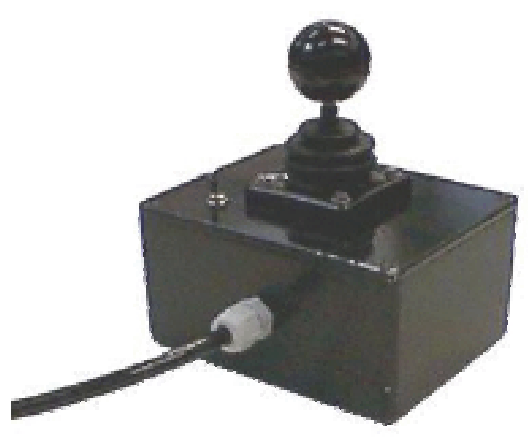

Figure 2.13 : Manus Joystick Controller

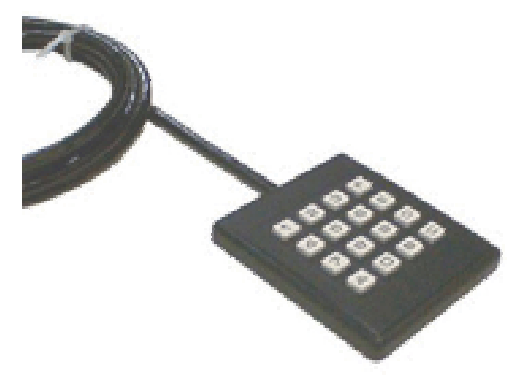

Figure 2.14 : Manus Keyboard Controller

\subsubsection{The Raptor}

Another production WMRA is the Raptor [Applied Resources, Inc.], which mounts the robotic arm to the right side of the wheelchair. This manipulator has four degrees of freedom plus a planar gripper and can be seen mounted to a power wheelchair in Figure 2.15. The arm is directly controlled by the user by either a joystick or 10-button controller. Because the Raptor does not have encoders to provide feedback to the controller, the manipulator cannot be pre-programmed in the fashion of industrial robots. This compromise was done to minimize overall system cost and make the product more readily available to the public. The simplicity of the Raptor arm and its controller allows it to be one-half the cost of the Manus arm. 


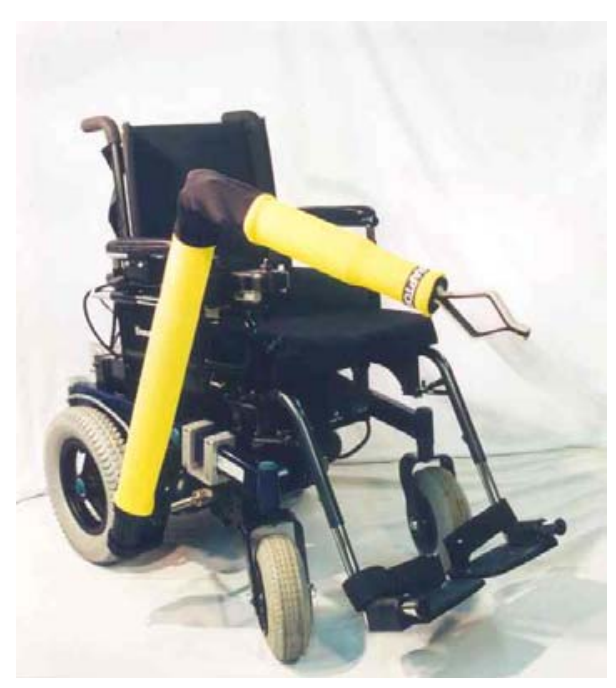

Figure 2.15 : Raptor Arm

\subsubsection{Side Mount}

The Raptor is a side-mounted arm. The primary joint motor of the Raptor is an exposed gear motor which must be mounted onto the frame under the seat of the wheelchair. In the specific application that was used, the motor is positioned slightly in front of the operator's waist. The Raptor side-mount is partially hidden underneath the chair. When the arm is not in use, the Raptor arm can be stowed relatively inconspicuously.

Similar in design to front-mounted manipulators, the side-mounted manipulators also have drawbacks. One significant problem with a side-mount robotic arm is that it increases the width of the power wheelchair. With the side-mount located lower than the armrest (under the wheelchair), the arm will always add at least the width of the first link to the width of the wheelchair. This makes it even more difficult to for the operator to maneuver through doorways and tight hallways ${ }^{9}$. This exacerbates mobility problems already encountered with power wheelchairs.

The side mount requires longer link lengths than a front mounted arm, to allow for manipulation of objects in front of the power wheelchair. These increased link lengths require larger and more powerful motors and gear-heads to move and stabilize the links' 
actuation. These factors often increase the weight and cost of designing arms for this application. The Raptor link geometry cannot be changed for specific applications.

\subsubsection{Open Loop Control}

An open loop controller places a human directly in the loop of controlling the arm. The operator continuously directs the arm into its final position. This type of system is inherently tolerant of positioning errors from a variety of causes. These errors may be specific to the robotic device such as play in the motors, gears, bearings or compliance

within the links due to loading or environmental conditions such as thermal effects, wind, and movement of the base with respect to the reference frame.

The open loop controller can correct for various types of positioning error because the operator continuously updates its position or the arm during the manipulation. The operator indirectly considers the sum of all the errors and moves the arm according to the actual perceived position of the end-effector.

Robotic arms with open loop control require higher levels of concentration and eye-hand coordination from the operator than closed loop systems. This may be more taxing for the operator and can limit the use of the assistive robotic device. Open loop systems are unable to make precisely reproducible motions.

A robotic system using an open loop controller may be much simpler by not requiring encoders to determine position or complex controllers. This trade-off allows for a cost-effective design. The Raptor exclusively employs an open loop control scheme. Typically these open loop controllers are driven one joint at a time in order to simplify the controller. 


\section{Chapter Three}

\section{Procedure for Kinematic Analysis}

\subsection{Determination of Workspace}

A workspace has been chosen which reflects specific requirements of individuals with disabilities 202122 .

Horizontal planes $(\mathrm{x}-\mathrm{y})$ were chosen with respect to the floor as the vertical axis $\mathrm{z}=$ 0 . The origin of the user-coordinate system is 31.8 " above the floor and all values given are referenced above the floor. A value of 2 " above a given plane was required in order to give room for the manipulator to reach an object. The value in parenthesis is the z-axis height with respect to the user coordinate system (farthest forward-most point between the armrests) can be seen in Figure 3.1.

1. Small objects on the floor: $2 "(-29.8 ”)$

2. Larger light objects on the floor: 9" (-22.8”)

3. Height of electric socket: 18 " (-13.8”)

4. Low coffee table: 26 " (-5.82")

5. Height of standard table and door knob: 31 " (-0.8”)

6. Kitchen counter top: 38 " (6.18”)

7. Wall-mounted light switch: 50" (18.2”)

8. Low shelf above kitchen counter top 56" (24.18”) 


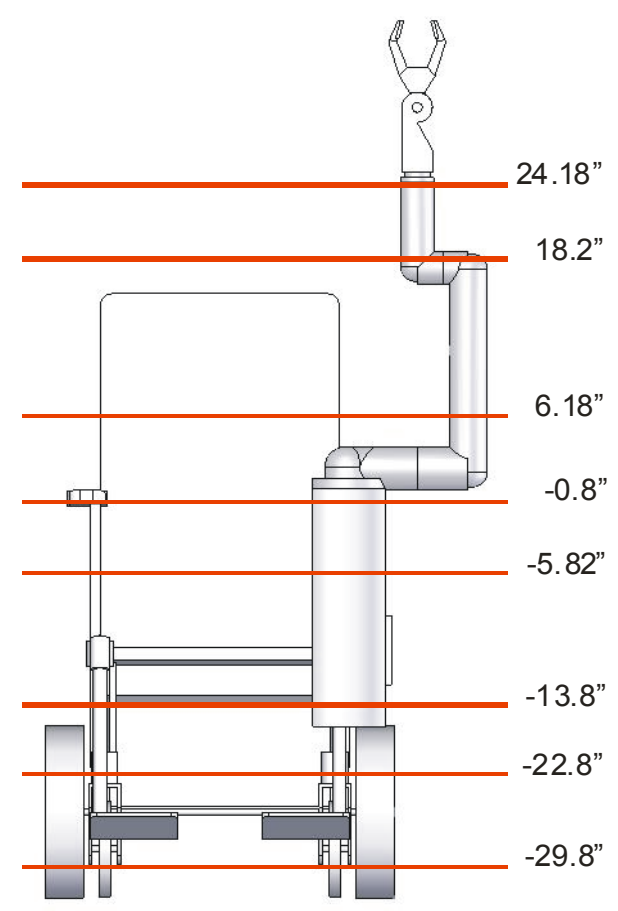

Figure 3.1 : Workspace Horizontal Planes

There are three horizontal lines that are also used in determining the manipulability measure. These are slightly above the lap of the operator and move from the upper tip of the wheelchairs armrests. These would be $(0, y, 0)$

Intersecting each of these horizontal (x-y) planes are vertical planes $(y-z)$ which reflect objects directly on axis with the wheelchair (as if the operator was driving straight forward). These are distances in front of the operator based on the frame reference that the top-most intersection of the tip of the arm rest is the origin. The distances are in the $\mathrm{x}$ axis of the user coordinate system (farthest forward-most point between the armrests) can be seen in Figure 3.2.

Starting from the farthest point and working toward the operator is described as follows. 
1. 2" in front of the footrest of the power wheelchair 27.54 ". This is the primary reference $x-z$ plane.

2. 14.04" in front of the operator. This is 13.5 " behind the first $x-z$ plane.

3. 6.75 " in front of the operator. This is half the distance of the 13.5 " grid.

4. 0.54 " is front of the operator. This is 13.5 " behind the second $x-z$ plane.

5. The $x-z$ plane at the origin of the user reference plane.

6. The x-z plane that reflects the mouth of the operator. 4" behind the origin.

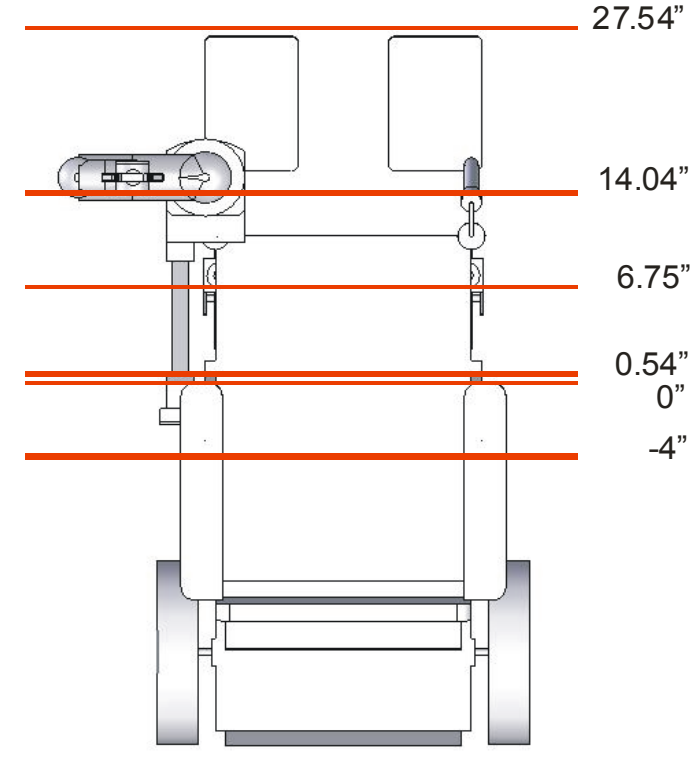

Figure 3.2 : Workspace Vertical Planes

Finally to create the individual points a third plane $(\mathrm{x}-\mathrm{z})$ is defined. The plane located at the origin separates the chair into two lateral halves and is shown in Figure 3.3. The wheelchair used for the analysis is 27 " wide including the width of the drive wheels. The $\mathrm{y}$ axis in the user frame of reference is positive moving from the body to the right hand extended out along the arm.

1. The plane intersecting the origin.

2. 13.5" from the origin toward the mounted arm. 
3. 23.5" from the origin toward the mounted arm. This represents the 10" from the outermost edge of the wheels.

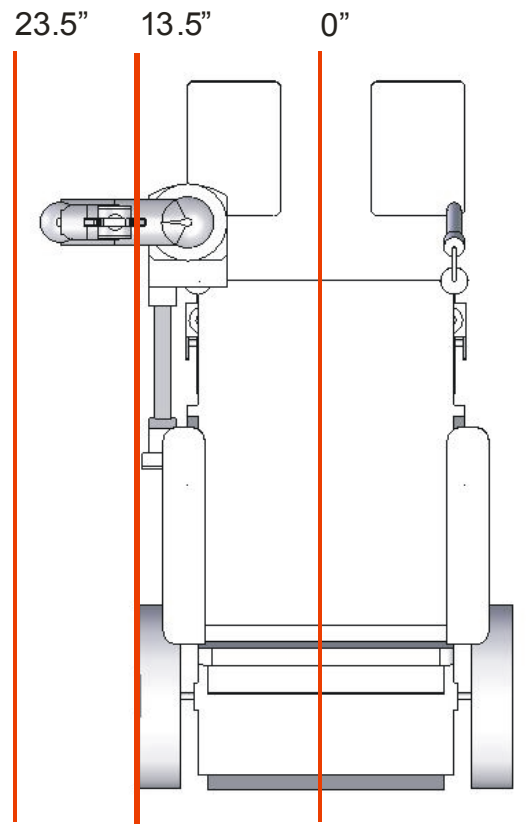

Figure 3.3 : Workspace Vertical Planes

\subsection{Denavit - Hartenberg Parameters}

The Denavit - Hartenberg parameters ${ }^{23}$ are a method of analyzing robotic manipulators that was first introduced in 1955. This technique allows robotics researchers an approach to standardize robotics nomenclature and to create an easy method to consider link arrangements in robotic manipulators. Each joint angle is analyzed separately with links separating each joint. A link is defined only as a rigid body that maintains a relationship between two adjacent joint axes within a manipulator. Joints in robotic mechanisms may be single dimensional such as rotational (revolute) or prismatic (linear extension). Of the two previous joint types the revolute joint is by far the most common.

Multidimensional (two and three dimension) joints could be cylindrical, screw, planar, or spherical. Due to the increased complexity of these types of joints they are not used as often as revolute or prismatic joints. When analyzing a joint of $\mathrm{n}$ dimensions with 
the Denavit-Hartenberg (D-H) parameter method the joint is broken into $\mathrm{n}$ joints with one degree of freedom connected to $\mathrm{n}-1$ links of zero links.

The Denavit-Hartenberg rules provide a guide for locating coordinate systems on each link of a multi-link kinematic chain. By following the D-H rules, the homogeneous transformations between adjacent links are defined. In order to use the D-H parameter method the parameters must be properly used. There are four parameters used in manipulator analysis. Three are fixed and are purely geometric these are the link twist, the link length, and the last is the link offset. The final parameter is variable and it is the joint angle. Within the nomenclature variable i refer to the link number.

The link length $\left(a_{i-1}\right)$ is the length of a line that is mutually orthogonal to the previous joint axis (i-1) and the next joint axis (i). The link twist $\left(\alpha_{1-1}\right)$ defines the relative location of the two joint axis. The link twist is determined by creating a plane which is normal to the previously mentioned mutually perpendicular line and projection of both axes onto this plane. The angle measured from link i-1 and link i (using a right hand rule for angle determination) is the link twist. The third parameter is link offset $\left(\mathrm{d}_{\mathrm{i}}\right)$ which is the distance along the common axis from one link to the following link. The final parameter is the joint angle $(\theta \mathrm{\imath})$.

The Figure 3.4 shows the relationships between link number (i), link length $\left(a_{i-1}\right)$, link twist $\left(\alpha_{\imath-1}\right)$, link offset $\left(d_{i}\right)$, and joint angle $(\theta i)$. 


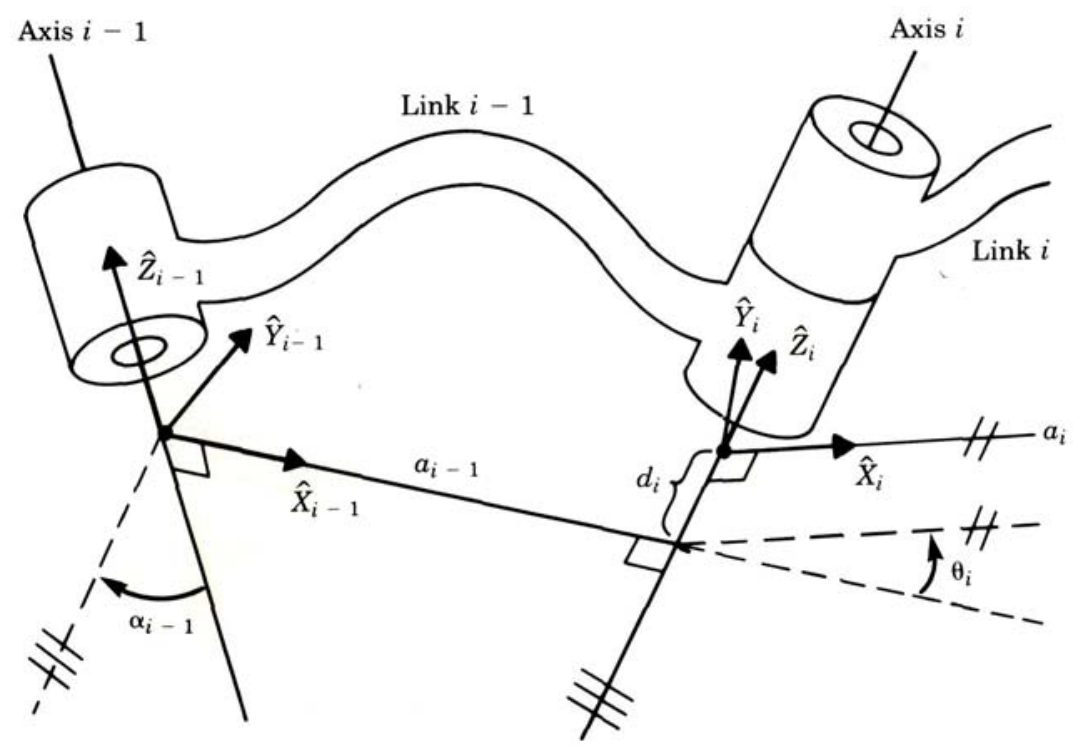

Figure 3.4 : D-H Parameter Link Parameters

The Link Transformation equation relates all of the D-H parameters together. It can be seen that each parameter is used once and in a specific order within the link transformation equation. This equation shown below and contain two rotations and two translations in a specific order. The first rotation is done about the $\mathrm{x}$ axis by the amount of the link twist $\left(\alpha_{1-1}\right)$, next there is a translation about the $\mathrm{x}$ axis by an amount of the link length $\left(\mathrm{a}_{\mathrm{i}-1}\right)$

$$
{ }_{i}^{i-1} T=R_{X}\left(\alpha_{i-1}\right) D_{X}\left(a_{i-1}\right) R_{Z}\left(\theta_{i}\right) D_{Z}\left(d_{i}\right)
$$

Equation 3.1

Equation 3.1 relates the order with which the rotation and translations must be accomplished in order to use the D-H parameter table is shown in.

$$
{ }_{i}^{i-1} T=\left[\begin{array}{cccc}
c \theta_{i} & -s \theta_{i} & 0 & a_{i-1} \\
s \theta_{i} c \alpha_{i-1} & c \theta_{i} c \alpha_{i-1} & -s \alpha_{i-1} & -s \alpha_{i-1} d_{i} \\
s \theta_{i} s \alpha_{i-1} & c \theta_{i} s \alpha_{i-1} & c \alpha_{i-1} & c \alpha_{i-1} d_{i} \\
0 & 0 & 0 & 1
\end{array}\right]
$$


The individual link transformations can be determined by using D-H parameters and Equation 3.2.

\subsection{Jacobian Matrix}

The Jacobian is a multidimensional form of the derivative. Because we are working with a three degree of freedom manipulator there are three separate equations that define the positional matrix (forward kinematics).

Let us consider that we have three functions, each comprised of three independent variables. This is equivalent to the three position vectors that are all functions of the three joint angles. In our case the $\mathrm{y}$ variables represent position and the $\mathrm{x}$ variables represent the joint angles. The functions $f_{n}$ are shown in Equation 3.3 represent the position vector from within the final transformation matrix of the manipulator.

$$
\begin{aligned}
& \mathrm{y}_{1}=\mathrm{f}_{1}\left(\mathrm{x}_{1}, \mathrm{x}_{2}, \mathrm{x}_{3}\right) \\
& \mathrm{y}_{2}=\mathrm{f}_{2}\left(\mathrm{x}_{1}, \mathrm{x}_{2}, \mathrm{x}_{3}\right) \\
& \mathrm{y}_{3}=\mathrm{f}_{3}\left(\mathrm{x}_{1}, \mathrm{x}_{2}, \mathrm{x}_{3}\right)
\end{aligned}
$$

These functions can also be expressed in vector notation by Equation 3.4.

$$
\mathrm{Y}=\mathrm{F}(\mathrm{X})
$$

Equation 3.4

Using the chain rule and differentiating we can determine the differentials of $y_{i}$ as a function of $x_{j}$ the previous function is shown in Equation 3.5. 


$$
\begin{aligned}
& \delta \mathrm{y}_{1}=\frac{\partial \mathrm{f}_{1}}{\partial \mathrm{x}_{1}} \delta \mathrm{x}_{1}+\frac{\partial \mathrm{f}_{1}}{\partial \mathrm{x}_{2}} \delta \mathrm{x}_{2}+\frac{\partial \mathrm{f}_{1}}{\partial \mathrm{x}_{3}} \delta \mathrm{x}_{3} \\
& \delta \mathrm{y}_{2}=\frac{\partial \mathrm{f}_{2}}{\partial \mathrm{x}_{1}} \delta \mathrm{x}_{1}+\frac{\partial \mathrm{f}_{2}}{\partial \mathrm{x}_{2}} \delta \mathrm{x}_{2}+\frac{\partial \mathrm{f}_{2}}{\partial \mathrm{x}_{3}} \delta \mathrm{x}_{3} \\
& \delta \mathrm{y}_{3}=\frac{\partial \mathrm{f}_{3}}{\partial \mathrm{x}_{1}} \delta \mathrm{x}_{1}+\frac{\partial \mathrm{f}_{3}}{\partial \mathrm{x}_{2}} \delta \mathrm{x}_{2}+\frac{\partial \mathrm{f}_{3}}{\partial \mathrm{x}_{3}} \delta \mathrm{x}_{3}
\end{aligned}
$$

Equation 3.5

To simply the previous equation we can again place it into vector notation in Equation 3.6.

$$
\delta \mathrm{Y}=\frac{\partial \mathrm{F}}{\partial \mathrm{X}} \delta \mathrm{X}
$$

Equation 3.6

This equation is a $3 \times 3$ matrix and is referred to as the Jacobian. It should be noted that if the functions $f_{1}(X)$ through $f_{3}(X)$ are nonlinear then their partial derivatives are a function of $\mathrm{x}_{\mathrm{i}}$. This equation can then be shown in Equation 3.7

$$
\delta \mathrm{Y}=\mathrm{J}(\mathrm{X}) \delta \mathrm{X}
$$

Equation 3.7

Finally by dividing by the differential time element the Jacobian becomes a method of mapping velocities in $\mathrm{X}$ to velocities in $\mathrm{Y}$.

$$
\delta \dot{\mathrm{Y}}=\mathrm{J}(\mathrm{X}) \delta \dot{\mathrm{X}}
$$

Equation 3.8

$\mathrm{J}(\mathrm{X})$ is a linear transformation that changes with time. Hence the Jacobian shown in Equation 3.8 is a time-varying linear transformation.

The use of the Jacobian in Robotics relates joint velocities to Cartesian velocity at the tip of the gripper. In a Jacobian matrix the number of rows indicate the number of 
degrees of freedom in the Cartesian space being considered and the number of columns equals the number of joints in the manipulator arm. It should be noted that there is an instantaneous linear relationship between joint angle rates and manipulator tip velocities. This relationship is used in the determination of joint angles from positional input is applied in the inverse kinematic program used to determine joint angles from positional input arguments.

The relationship between joint velocity and Cartesian manipulator tip velocities in Equation 3.9 relies on the requirement that the Jacobian velocity be invertible. A matrix that is singular is not invertible. In order for the Jacobian to be used in this application it must be non-singular and thus invertible.

$$
\dot{\Theta}=J^{-1}(\Theta) V
$$

Equation 3.9

Most manipulators have values for their joint angles where the Jacobian becomes singular. These points are referred to as singularities of the mechanism or often shorted to singularities. All robotic manipulators have singularities at the limit of their workspace. Additionally there are singularities within the workspace of manipulators as well. These are referred to as internal singularities. From this we can see that there exist two types of singularities:

Workspace boundary singularities occur when the manipulator is fully extended or folded upon itself so that the end effector is sufficiently near the boundary of the workspace. The other type of singularity is a Workspace interior singularity. These are usually away from the workspace boundary but generally occur where two or more joint axes line up. At a singularity the manipulator looses one or more degrees of freedom in Cartesian space making the movement of the end effector impossible.

\subsection{Manipulability Ellipsoid}

A concept known as the manipulability ellipsoid will be introduced as well as the volume of the ellipsoid the manipulability measure. In the end a total evaluation of any 
manipulator system would require the analysis of many factors such as the volume of the workspace, ease of use, speed, precision and accuracy etc. The manipulability measure is the absolute value of the determinate of the Jacobian matrix of the positional sub-matrix of the final transformation matrix of the manipulators arm reference frame.

We will consider a manipulator with three degrees of freedom. This is the case with both manipulators being studied. The three joint variables will be denoted as a $3 \times 1$ vector and describe the position of the end effector. The kinematic relation between $\mathbf{q}$ and $\mathbf{r}$ is shown in Equation 3.10.

$$
\mathbf{r}=\mathrm{f}_{\mathrm{r}}(\mathrm{q})
$$

The relation between the velocity vector $\mathbf{v}$ corresponding to $\mathbf{r}$ and the joint velocity is shown in Equation 3.11.

$$
\mathbf{v}=\mathbf{J}(\mathbf{q}) \dot{q}
$$

Equation 3.11

where $\mathbf{J}(\mathbf{q})$ is the Jacobian matrix in Equation 3.11

If we consider the set of all possible joint velocities and the resultant end effector velocities

$$
\|\dot{\mathrm{q}}\|=\sqrt{\left(\dot{\mathrm{q}}_{1}^{2}+\dot{\mathrm{q}}_{2}^{2}+\dot{\mathrm{q}}_{3}^{2}\right)}
$$

In the Equation 3.12, the value to the left of the equation, must be less than or equal to unity. This is the manipulability ellipsoid with the major axis of the ellipsoid being the 
vector which will allow for the greatest end effector velocity and the minor axis of the ellipsoid will be vector which gives the lowest possible end effector velocity. In the case being analyzed the manipulability ellipsoid will have three axes. In manipulators with $\mathrm{m}$ links the manipulability ellipsoid will have $m$ axes.

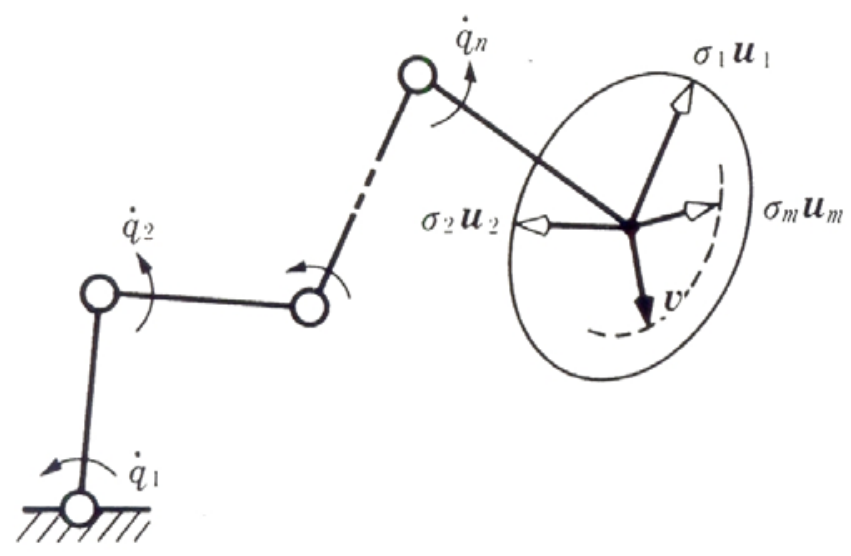

Figure 3.5 : Manipulability Ellipsoid

In the special case where all the ellipsoid axes are equal the ellipsoid will actually be spherical. At the special case where the manipulability ellipsoid is spherical the end effector can move in any direction with the same maximum velocity. The larger the size of the ellipsoid the faster the end effector can move.

One possible method of analysis is to determine the volume of the ellipsoid. This is computationally straight forward and is defined in Equation 3.13 by $\mathbf{c}_{\mathbf{m}} \mathbf{w}$ where:

$$
\begin{array}{cl}
\mathrm{c}_{\mathrm{m}}= \begin{cases}2 \pi^{\mathrm{m} / 2} /[2 \cdot 4 \cdot 6 \cdots \cdot(\mathrm{m}-2) \cdot \mathrm{m}] & \text { if } \mathrm{m} \text { is odd } \\
2(2 \pi)^{(\mathrm{m}-1) / 2} /[1 \cdot 3 \cdot 5 \cdots \cdots(\mathrm{m}-2) \cdot \mathrm{m}] & \text { if } \mathrm{m} \text { is even }\end{cases} & \text { Equation } 3.13 \\
\mathrm{w}=\sigma_{1} \sigma_{2} \sigma_{3} &
\end{array}
$$

The value of $\mathbf{c}_{\mathbf{m}}$ is constant when $\mathrm{m}$ is fixed. This is the case, $\mathrm{m}=3$, with the manipulators being studied. Because the value of $\mathbf{c}_{\mathbf{m}}$ is constant we can see that the 
volume of the ellipsoid is proportional to the value of $\mathbf{w}$. We refer to $\mathbf{w}$ as the manipulability measure. The manipulability measure has specific properties that allow us to define it readily from the Jacobian and known joint angles. First in the broadest sense the manipulability measure shown is shown in Equation 3.14:

$$
\mathrm{W}=\sqrt{\operatorname{det} J(q) J_{\mathrm{T}}(q)}
$$

Equation 3.14

And more specifically to our application where the manipulator is non-redundant the previous equation reduces to Equation 3.15.

$$
\mathrm{w}=|\operatorname{det} \mathrm{J}(\mathrm{q})|
$$

This shows that at a singular configuration the value of $\mathrm{w}$ approaches or equals zero. Hence the value of the manipulability measure, which is the volume of the manipulability ellipsoid, will be equal to the determinate of the Jacobian.

\subsection{Inverse Kinematic Program}

A method for determining the joint angles of each robot arm was required in order to determine the manipulability measure and to verify it within the solid model in Solid WORKS $^{\circledR}$. An overview of the program and the methods required to operate its subroutines is as follows.

In essence, the manipulability measure is the absolute value of the determinate of the Jacobian matrix of the positional sub-matrix of the final transformation matrix of the manipulators arm reference frames. It is this value that is used as the main 
The Jacobian matrix is derived from the $1 \times 3$ positional matrix within the final transformation matrix of the arm. The transformation matrix is created through the judicious application of the DH-parameters using the appropriate formulae.

One challenge is to determine the joint angles of the robotic arm for a given point in 3-D space. This requires the use of a method of reverse kinematics. The reverse kinematics of the robotic arms was determined with a program in MatLAB.

The program shown in Figure 3.6 uses the Jacobian matrix in a numerical methods approach subdividing the positional difference from the start position of the gripper and the desired goal position into several discrete goals. These discrete goal positions were entered sequentially entered into the Jacobian.

The output of the Jacobian is a matrix containing the incremental change of joint angles required to obtain the new position. The joint angle change is added to the previous joint angle and this new value is input into the forward kinematics of the transformation matrix. The actual position is compared to the desired discrete position and if it is below an error value than the next position is computed. If the error is too great the final error position is subtracted from the desired position and recomputed into the Jacobian and the process repeats the loop.

In the inverse kinematic solution there are four subroutines along with the main program. The subroutines are called by the main program to execute additional steps. 


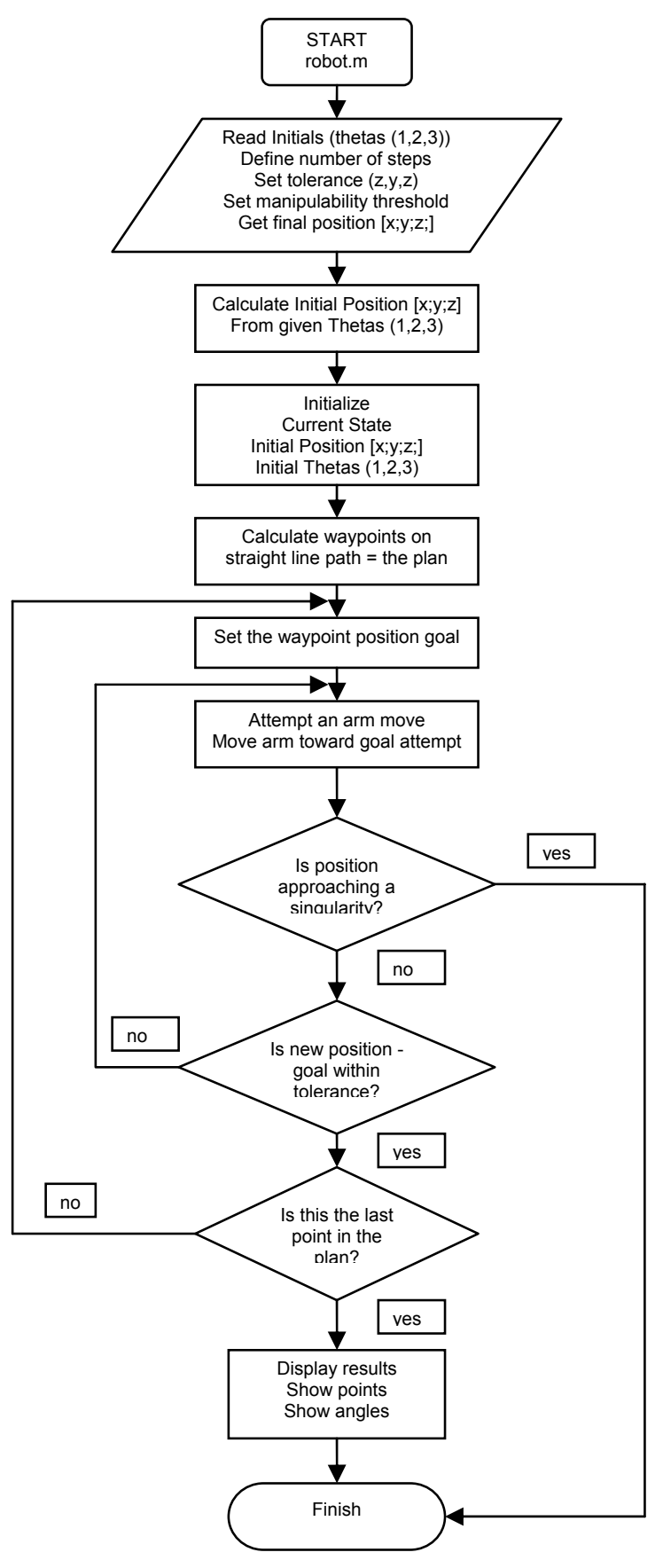

Figure 3.6 : Program Flowchart 
The main program is called robot.m and is a MatLAB file which has been extensively commented for ease in comprehension. The code for robot.m and the five subroutines that it calls upon can be found in the Appendix B. The subroutines are listed in the order that they are called upon.

\subsection{Procedure for Analysis}

The evaluation of WMRAs will encompass three steps. First is the creation of a procedure for the kinematic analysis of any robotic arm. Second is the evaluation of two commercially available manipulators (Manus and Raptor). And third are the design recommendations or insights gained from the second step.

In order to create a procedure for the kinematic analysis of WMRAs it is necessary to separate the process into a series of steps. More specifically the procedure followed these steps:

1. Create a Denavit - Hartenberg parameter table and transformation matrices for the manipulator to be measured.

2. Create link transformations for the manipulator.

3. Determine the Jacobian Matrix for the manipulator.

4. Model the manipulator and a generic power wheelchair in Solid WORKS ${ }^{\circledR}$ so that angle and joint relationships can be shown graphically.

5. Pick a series of points (grid) surrounding the wheelchair / arm assembly. These points have specific applications in rehabilitation engineering.

6. Create a computer program using a numerical methods approach to determine the joint angles of the arm for a given point in the workspace. The joint angles are then used to determine the manipulability of the arm for the given point.

7. Plot and compare the normalized manipulability measures for each arm. Verify that the joint angle provided by the inverse kinematics program correspond to positions in the model space. 


\section{Chapter Four Analysis Results}

4.1 Evaluation of the Manus:

This is the analysis of the Manus system. An operating unit was not available to test directly. Therefore, specifications from the manufacturer, technical illustrations and photographs of the system were used to create the solid model. It was reproduced as faithfully as possible with the provided information.

The figure below shows the frames of reference for the power wheelchair and the Manus. The Manus in Figure 4.1 is shown in its fully lowered position. This position was chosen because it allows the manipulator access to the floor. It is possible that the manipulability measures would be higher when reaching into cabinets if the Z-lift mechanism were used. The reference frames are important in understanding the relationships shown in the D-H Parameter tables. 


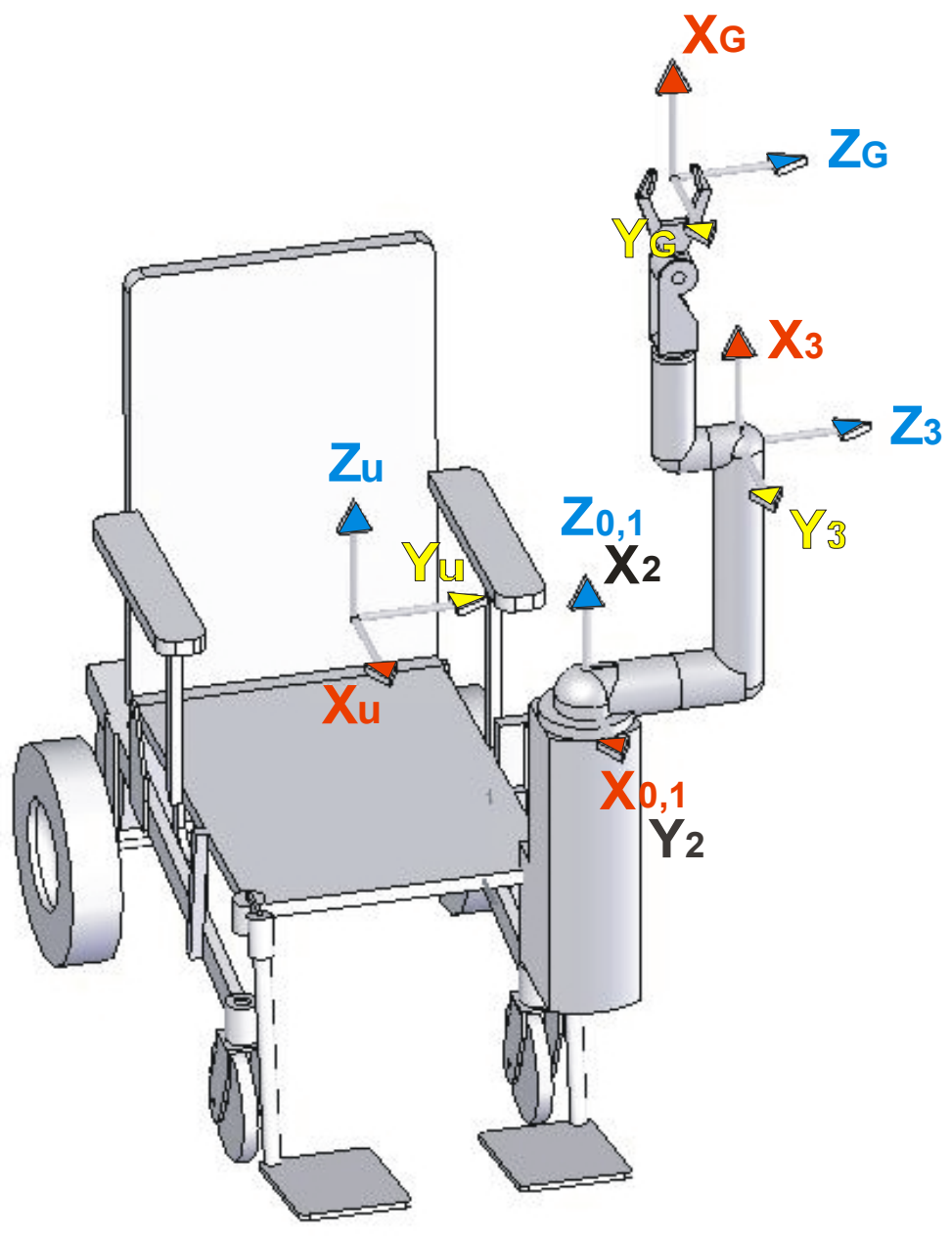

Figure 4.1 : Manus Reference Frames

The transformations for each joint with respect to the previous joint are shown below. The nomenclature for each matrix is as follows.

$\mathrm{T}$

wrt

Number (pre \& post)
Transformation Matrix

With Respect To

Corresponds to the frame of reference 
The D-H Parameters for the Manus with 3 DOF are outlined in Table 4.1. The table shows the relationships between link number (i), link twist $\left(\alpha_{1-1}\right)$, link length $\left(a_{i-1}\right)$, link offset $\left(\mathrm{d}_{\mathrm{i}}\right)$, and joint angle $(\theta \mathrm{l})$.

Table 4.1 : D-H Parameters for Manus

\begin{tabular}{|l|l|l|l|l|}
\hline $\mathrm{i}$ & $\alpha_{\mathrm{l}-1}$ & $\mathrm{a}_{\mathrm{i}-1}$ & $\mathrm{~d}_{\mathrm{i}}$ & $\theta \mathrm{t}$ \\
\hline 1 & $0^{\circ}$ & 0 & 0 & $\theta_{1}$ \\
\hline 2 & $-90^{\circ}$ & 0 & 0 & $\left(\theta_{2}-90^{\circ}\right)$ \\
\hline 3 & $0^{\circ}$ & 15.75 & 9.20 & $\theta_{3}$ \\
\hline 4 & $0^{\circ}$ & 18.77 & -3.94 & 0 \\
\hline
\end{tabular}

Figure 4.1 is used to create the D-H Parameters shown in Table 4.1. These parameters are entered into Equation 3.2 to achieve each respective frame transformation. The transformation matrix which relates frame 1 with respect to frame 0 is shown in Equation 4.1

$$
\text { T1wrt0 }:=\left(\begin{array}{cccc}
\cos (\theta 1) & -\sin (\theta 1) & 0 & 0 \\
\sin (\theta 1) & \cos (\theta 1) & 0 & 0 \\
0 & 0 & 1 & 0 \\
0 & 0 & 0 & 1
\end{array}\right)
$$

The transformation matrix which relates frame 2 with respect to frame 1 is shown in the Equation 4.2.

$$
\mathrm{T} 2 \mathrm{wrt1}:=\left(\begin{array}{cccc}
\sin (\theta 2) & \cos (\theta 2) & 0 & 0 \\
0 & 0 & 1 & 0 \\
\cos (\theta 2) & -\sin (\theta 2) & 0 & 0 \\
0 & 0 & 0 & 1
\end{array}\right)
$$

Equation 4.2

The transformation matrix which relates frame 3 with respect to frame 2 is shown in the Equation 4.3. 


$$
=\left(\begin{array}{cccc}
\cos (\theta 3) & -\sin (\theta 3) & 0 & 15.75 \\
\sin (\theta 3) & \cos (\theta 3) & 0 & 0 \\
0 & 0 & 1 & 9.20 \\
0 & 0 & 0 & 1
\end{array}\right)
$$

The transformation matrix which relates frame $G$, or the frame of the gripper, with respect to frame 3 is shown in Equation 4.4.

$$
\text { TGwrt3:=( }=\left(\begin{array}{cccc}
1 & 0 & 0 & 18.77 \\
0 & 1 & 0 & 0 \\
0 & 0 & 1 & -3.94 \\
0 & 0 & 0 & 1
\end{array}\right)
$$

Equation 4.4

A user frame $\{U\}$ is created to reflect a frame with correlation to the user. Equation 4.5 below shows the transformation matrix which defines the translational relationship between frame $0\{0\}$ with respect to the user frame $\{U\}$.

$$
\text { T0wrtU : }=\left(\begin{array}{cccc}
1 & 0 & 0 & 15.04 \\
0 & 1 & 0 & 9.97 \\
0 & 0 & 1 & 1.74 \\
0 & 0 & 0 & 1
\end{array}\right)
$$

The transformation matrices are multiplied together (Equation 4.6) to give the transform relating the end gripper position with respect to the user frame.

$$
\text { T0wrtU*T1wrt0*T2wrt1*T3wrt2*TGwrt3 = TGwrtU Equation } 4.6
$$

The final transformational matrix is shown in Equation 4.7. Due to the size of the transformation matrix and the constraints of page formatting, the matrix has been separated into columns one and two: 


$$
\left(\begin{array}{cc}
\cos (\theta 1) \bullet \sin (\theta 2) \bullet \cos (\theta 3)+\cos (\theta 1) \bullet \cos (\theta 2) \bullet \sin (\theta 3) & -\cos (\theta 1) \bullet \sin (\theta 2) \bullet \sin (\theta 3)+\cos (\theta 1) \bullet \cos (\theta 2) \bullet \cos (\theta 3) \\
\sin (\theta 1) \bullet \sin (\theta 2) \bullet \cos (\theta 3)+\sin (\theta 1) \bullet \cos (\theta 2) \bullet \sin (\theta 3) & -\sin (\theta 1) \bullet \sin (\theta 2) \bullet \sin (\theta 3)+\sin (\theta 1) \bullet \cos (\theta 2) \bullet \cos (\theta 3) \\
\cos (\theta 2) \bullet \cos (\theta 3)-\sin (\theta 2) \bullet \sin (\theta 3) & -\cos (\theta 2) \bullet \sin (\theta 3)-\sin (\theta 2) \bullet \cos (\theta 3) \\
0 & 0
\end{array}\right)
$$

Equation 4.7

and then columns three and four:

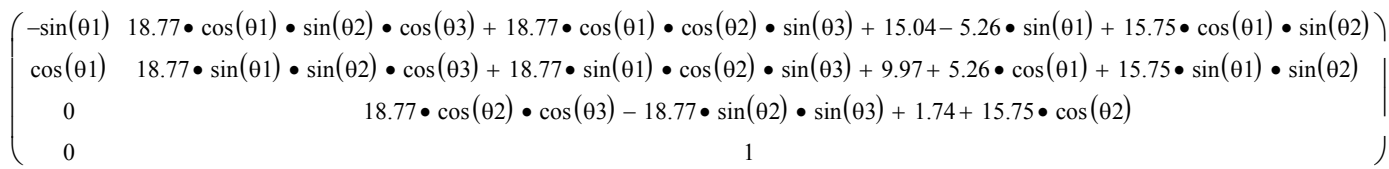

The position vector, a $3 \times 1$ matrix, is the first three rows of the final column of the final transformational matrix and is shown in Equation 4.8. This matrix is also the forward kinematic matrix. When the three joint angles are computed, the result is the position of the gripper in space with respect to the user frame $\{U\}$. From the forward kinematic matrix we can compute the Jacobian Matrix - the partial derivative of the positional matrix.

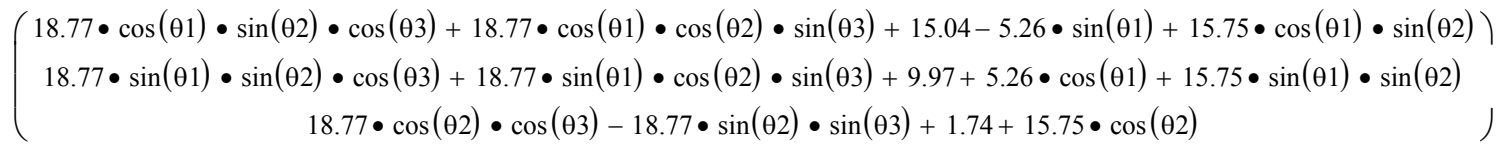

Equation 4.8

Equation 4.9 below is the Jacobian of the Manus. The matrix has been separated into three columns and is shown sequentially for ease of viewing.

\section{Column 1:}

$-18.77^{*} \sin (\mathrm{t} 1)^{*} \sin (\mathrm{t} 2)^{*} \cos (\mathrm{t} 3)-18.77^{*} \sin (\mathrm{t} 1)^{*} \cos (\mathrm{t} 2)^{*} \sin (\mathrm{t} 3)-5.26^{*} \cos (\mathrm{t} 1)-15.75^{*} \sin (\mathrm{t} 1)^{*} \sin (\mathrm{t} 2)$

$18.77^{*} \cos (\mathrm{t} 1)^{*} \sin (\mathrm{t} 2)^{*} \cos (\mathrm{t} 3)+18.77^{*} \cos (\mathrm{t} 1)^{*} \cos (\mathrm{t} 2)^{*} \sin (\mathrm{t} 3)-5.26^{*} \sin (\mathrm{t} 1)+15.75^{*} \cos (\mathrm{t} 1)^{*} \sin (\mathrm{t} 2)$ 
Column 2:

$18.77^{*} \cos (\mathrm{t} 1)^{*} \cos (\mathrm{t} 2)^{*} \cos (\mathrm{t} 3)-18.77^{*} \cos (\mathrm{t} 1)^{*} \sin (\mathrm{t} 2)^{*} \sin (\mathrm{t} 3)+15.75^{*} \cos (\mathrm{t} 1)^{*} \cos (\mathrm{t} 2)$

$18.77^{*} \sin (\mathrm{t} 1)^{*} \cos (\mathrm{t} 2)^{*} \cos (\mathrm{t} 3)-18.77^{*} \sin (\mathrm{t} 1)^{*} \sin (\mathrm{t} 2)^{*} \sin (\mathrm{t} 3)+15.75^{*} \sin (\mathrm{t} 1)^{*} \cos (\mathrm{t} 2)$

$-18.77^{*} \sin (\mathrm{t} 2)^{*} \cos (\mathrm{t} 3)-18.77^{*} \cos (\mathrm{t} 2)^{*} \sin (\mathrm{t} 3)-15.75^{*} \sin (\mathrm{t} 2)$

Column 3:

$$
\begin{gathered}
-18.77^{*} \cos (\mathrm{t} 1)^{*} \sin (\mathrm{t} 2)^{*} \sin (\mathrm{t} 3)+18.77^{*} \cos (\mathrm{t} 1)^{*} \cos (\mathrm{t} 2)^{*} \cos (\mathrm{t} 3) \\
-18.77^{*} \sin (\mathrm{t} 1)^{*} \sin (\mathrm{t} 2)^{*} \sin (\mathrm{t} 3)+18.77^{*} \sin (\mathrm{t} 1)^{*} \cos (\mathrm{t} 2)^{*} \cos (\mathrm{t} 3) \\
-18.77^{*} \cos (\mathrm{t} 2)^{*} \sin (\mathrm{t} 3)-18.77^{*} \sin (\mathrm{t} 2)^{*} \cos (\mathrm{t} 3)
\end{gathered}
$$

Values for the manipulability measure are plotted in both horizontal and vertical planes. The grid density in the analyzed workspace is greater in the $\mathrm{z}$ axis, which gives a greater number of points with which to observe trends and changes of the manipulability measure. There are four vertical axes and eight horizontal axes. 


\subsubsection{Vertical Planes}

In robotics, the term approach is used to describe reaching a point in space without regard for gripper orientation. In this thesis, the term approach and also access will be used in a similar fashion, indicating that the arm is capable of reaching that specific point, or in broader terms, the area specified. In order for a point to be defined as having access, it must have a manipulability measure of at least 100 . The maximum value of the manipulability measure in the data set was 7084.4 at point $[-4,-6.75,13.5]$.

Because of the 3-dimensional nature of the data to be diagrammed, a method for representing the relative value of the manipulability measure and a qualitative determination are shown in Figure 4.2. The size and color of the spheres are used to represent the manipulability measure as a percentage of the maximum manipulability measure computed.

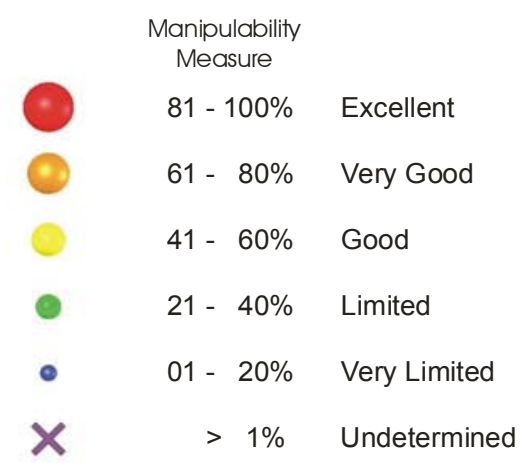

Figure 4.2 : Representation of the Manipulability Measure

Figure 4.3 through Figure 4.6 show the manipulability measures of the Manus for vertical planes within the defined workspace.

The Manus arm offered very good access to low cabinets and shelves in front of and to the far left corner of the workspace that has been defined. In Figure 4.3 the manipulability measure remained very good throughout the z-axis in this far left corner of the operator's workspace. A significant limitation was the very limited ability to grab 
objects from the floor directly in front of the operator's feet. The area of highest agility would be at least partially obscured by the robot base.

Table 4.2 : Data for $\mathrm{x}=27.54$ "

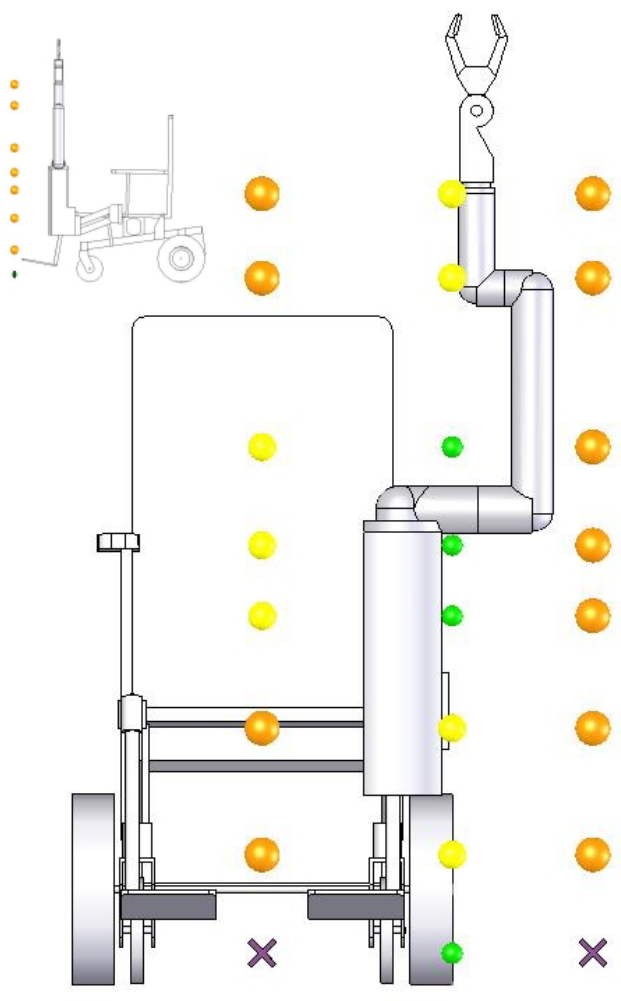

Figure 4.3 : Manus y-z Plane@x=27.54"

\begin{tabular}{|c|c|c|c|}
\hline $\mathbf{x}$ & $\mathbf{y}$ & $\mathbf{z}$ & $\mathbf{n}$ \\
\hline 27.54 & 0 & 24.18 & 0.64 \\
\hline 27.54 & 13.5 & 24.18 & 0.52 \\
\hline 27.54 & 23.5 & 24.18 & 0.72 \\
\hline 27.54 & 0 & 18.2 & 0.65 \\
\hline 27.54 & 13.5 & 18.2 & 0.49 \\
\hline 27.54 & 23.5 & 18.2 & 0.77 \\
\hline 27.54 & 0 & 6.18 & 0.53 \\
\hline 27.54 & 13.5 & 6.18 & 0.35 \\
\hline 27.54 & 23.5 & 6.18 & 0.69 \\
\hline 27.54 & 0 & -0.8 & 0.52 \\
\hline 27.54 & 13.5 & -0.8 & 0.33 \\
\hline 27.54 & 23.5 & -0.8 & 0.68 \\
\hline 27.54 & 0 & -5.82 & 0.56 \\
\hline 27.54 & 13.5 & -5.82 & 0.38 \\
\hline 27.54 & 23.5 & -5.82 & 0.71 \\
\hline 27.54 & 0 & -13.8 & 0.64 \\
\hline 27.54 & 13.5 & -13.8 & 0.48 \\
\hline 27.54 & 23.5 & -13.8 & 0.77 \\
\hline 27.54 & 0 & -22.8 & 0.61 \\
\hline 27.54 & 13.5 & -22.8 & 0.50 \\
\hline 27.54 & 23.5 & -22.8 & 0.66 \\
\hline 27.54 & 0 & -29.8 & 0.00 \\
\hline 27.54 & 13.5 & -29.8 & 0.22 \\
\hline 27.54 & 23.5 & -29.8 & 0.00 \\
\hline & & & \\
\hline
\end{tabular}

As the yz - plane approaches the manipulator base, a singularity creates a limitation of movement near the first rotational axis as shown in Figure 4.4. At the line of data points shown in Figure 4.4 that satisfy $y=13.5$ " and $y-z$ plane $x=14.04$ ", the manipulability measure becomes very low. With the threshold that was used for the program, these points were defined as unobtainable. 


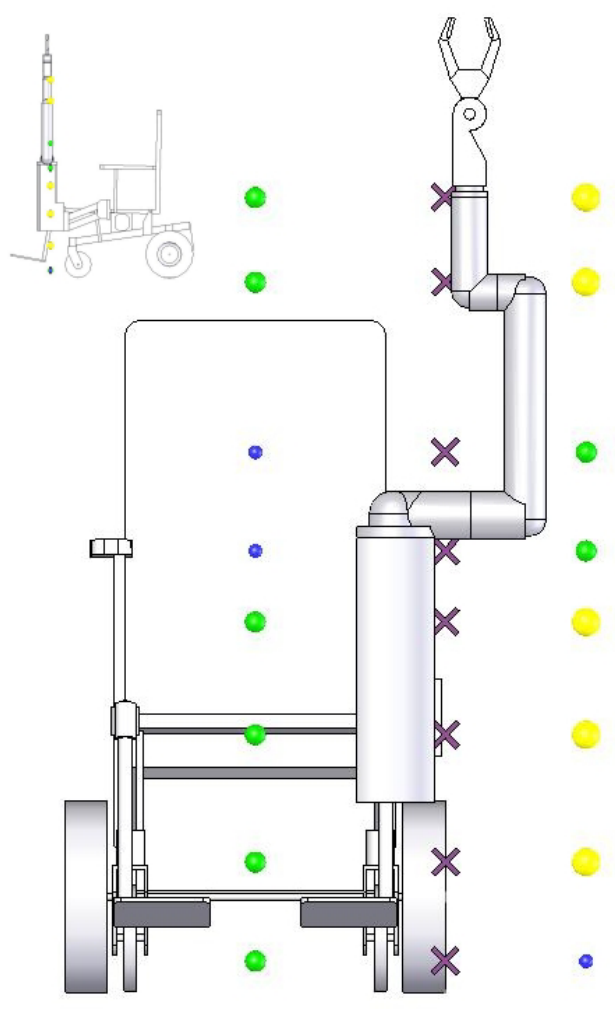

Figure 4.4 : Manus y-z Plane @ $\mathrm{x}=14.04 "$
Table 4.3 : Data for $\mathrm{x}=14.04 ”$

\begin{tabular}{|c|c|c|c|}
\hline $\mathbf{x}$ & $\mathbf{y}$ & $\mathbf{z}$ & $\mathbf{n}$ \\
\hline 14.04 & 0 & 24.18 & 0.37 \\
\hline 14.04 & 13.5 & 24.18 & 0.00 \\
\hline 14.04 & 23.5 & 24.18 & 0.54 \\
\hline 14.04 & 0 & 18.2 & 0.34 \\
\hline 14.04 & 13.5 & 18.2 & 0.00 \\
\hline 14.04 & 23.5 & 18.2 & 0.52 \\
\hline 14.04 & 0 & 6.18 & 0.19 \\
\hline 14.04 & 13.5 & 6.18 & 0.00 \\
\hline 14.04 & 23.5 & 6.18 & 0.38 \\
\hline 14.04 & 0 & -0.8 & 0.18 \\
\hline 14.04 & 13.5 & -0.8 & 0.00 \\
\hline 14.04 & 23.5 & -0.8 & 0.37 \\
\hline 14.04 & 0 & -5.82 & 0.23 \\
\hline 14.04 & 13.5 & -5.82 & 0.00 \\
\hline 14.04 & 23.5 & -5.82 & 0.41 \\
\hline 14.04 & 0 & -13.8 & 0.33 \\
\hline 14.04 & 13.5 & -13.8 & 0.00 \\
\hline 14.04 & 23.5 & -13.8 & 0.51 \\
\hline 14.04 & 0 & -22.8 & 0.37 \\
\hline 14.04 & 13.5 & -22.8 & 0.00 \\
\hline 14.04 & 23.5 & -22.8 & 0.53 \\
\hline 14.04 & 0 & -29.8 & 0.23 \\
\hline 14.04 & 13.5 & -29.8 & 0.00 \\
\hline 14.04 & 23.5 & -29.8 & 0.20 \\
\hline & & & \\
\hline
\end{tabular}

The Manus arm as shown in Figure 4.5 has very good access to objects along the side of the chair at the vertical plane of $x=6.75$ ". The only limitation to this side reach is approaching low objects on the floor. It should also be noted that the effect of the singularity shown in Figure 4.4 has not been completely eliminated. At the vertical line on the $\mathrm{yz}$ - plane at $\mathrm{y}=13.5$, the manipulability measure is very low from coffee table ( $\mathrm{z}$ $=-5.82 ")$ to kitchen countertop $(\mathrm{z}=6.18$ "). 


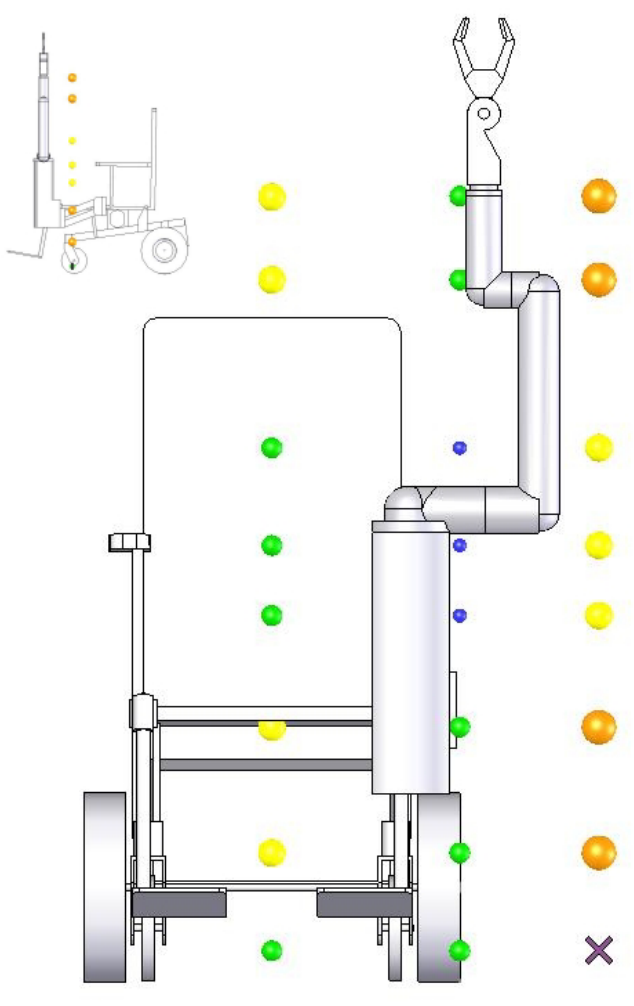

Figure 4.5 : Manus y-z Plane @ $\mathrm{x}=6.75 "$

Table 4.4 : Data for $\mathrm{x}=6.75$ "

\begin{tabular}{|c|c|c|c|}
\hline $\mathbf{x}$ & $\mathbf{y}$ & $\mathbf{z}$ & $\mathbf{n}$ \\
\hline 6.75 & 0 & 6.18 & 0.35 \\
\hline 6.75 & 13.5 & 6.18 & 0.14 \\
\hline 6.75 & 23.5 & 6.18 & 0.51 \\
\hline 6.75 & 0 & 24.18 & 0.52 \\
\hline 6.75 & 13.5 & 24.18 & 0.32 \\
\hline 6.75 & 23.5 & 24.18 & 0.64 \\
\hline 6.75 & 0 & 18.2 & 0.49 \\
\hline 6.75 & 13.5 & 18.2 & 0.28 \\
\hline 6.75 & 23.5 & 18.2 & 0.64 \\
\hline 6.75 & 0 & -0.8 & 0.33 \\
\hline 6.75 & 13.5 & -0.8 & 0.13 \\
\hline 6.75 & 23.5 & -0.8 & 0.51 \\
\hline 6.75 & 0 & -5.82 & 0.37 \\
\hline 6.75 & 13.5 & -5.82 & 0.18 \\
\hline 6.75 & 23.5 & -5.82 & 0.55 \\
\hline 6.75 & 0 & -13.8 & 0.47 \\
\hline 6.75 & 13.5 & -13.8 & 0.27 \\
\hline 6.75 & 23.5 & -13.8 & 0.64 \\
\hline 6.75 & 0 & -22.8 & 0.50 \\
\hline 6.75 & 13.5 & -22.8 & 0.32 \\
\hline 6.75 & 23.5 & -22.8 & 0.61 \\
\hline 6.75 & 0 & -29.8 & 0.25 \\
\hline 6.75 & 13.5 & -29.8 & 0.21 \\
\hline 6.75 & 23.5 & -29.8 & 0.00 \\
\hline & & & \\
\hline
\end{tabular}

Access to high shelves and counters increased as the operator approached them from the left side. Values of the manipulability measure in Figure 4.6 were near optimum for reaching objects when the operator aligned the goal parallel to the seat of the chair at the yz plane of $x=0.54$ ". It can also be seen in the following figures that the measure was near maximum between the values of $x$ from 0 " to -4 ". 


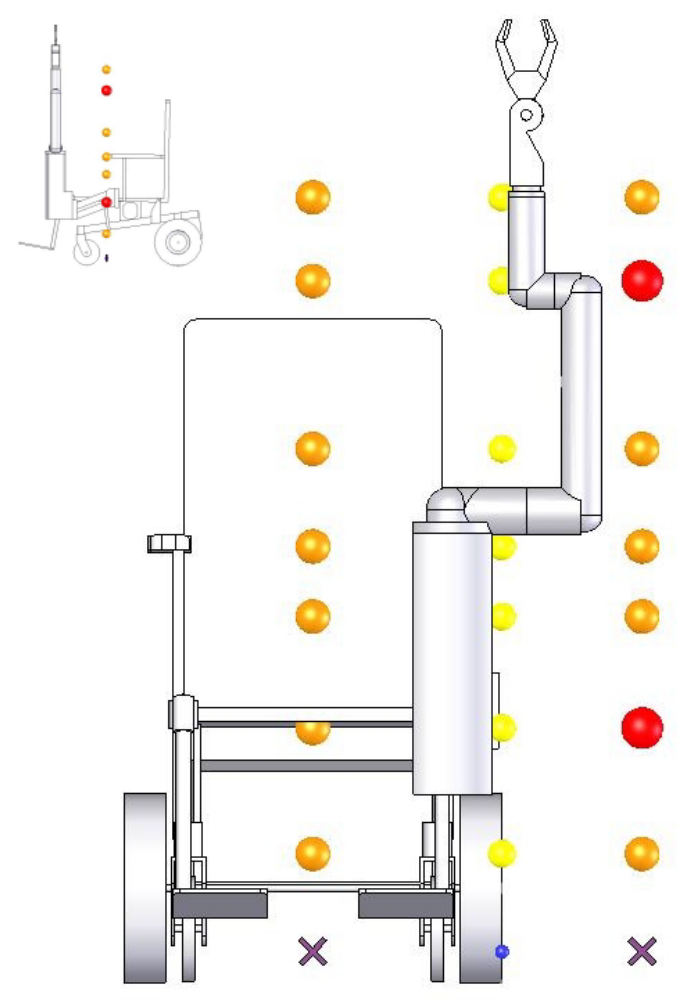

Figure 4.6 : Manus y-z Plane @ $\mathrm{x}=0.54 "$
Table 4.5 : Data for $\mathrm{x}=0.54 "$

\begin{tabular}{|c|c|c|c|}
\hline $\mathbf{x}$ & $\mathbf{y}$ & $\mathbf{z}$ & $\mathbf{n}$ \\
\hline 0.54 & 0 & 24.18 & 0.70 \\
\hline 0.54 & 13.5 & 24.18 & 0.60 \\
\hline 0.54 & 23.5 & 24.18 & 0.75 \\
\hline 0.54 & 0 & 18.2 & 0.73 \\
\hline 0.54 & 13.5 & 18.2 & 0.59 \\
\hline 0.54 & 23.5 & 18.2 & 0.83 \\
\hline 0.54 & 0 & 6.18 & 0.63 \\
\hline 0.54 & 13.5 & 6.18 & 0.46 \\
\hline 0.54 & 23.5 & 6.18 & 0.78 \\
\hline 0.54 & 0 & -0.8 & 0.62 \\
\hline 0.54 & 13.5 & -0.8 & 0.45 \\
\hline 0.54 & 23.5 & -0.8 & 0.77 \\
\hline 0.54 & 0 & -5.82 & 0.66 \\
\hline 0.54 & 13.5 & -5.82 & 0.49 \\
\hline 0.54 & 23.5 & -5.82 & 0.79 \\
\hline 0.54 & 0 & -13.8 & 0.72 \\
\hline 0.54 & 13.5 & -13.8 & 0.58 \\
\hline 0.54 & 23.5 & -13.8 & 0.83 \\
\hline 0.54 & 0 & -22.8 & 0.65 \\
\hline 0.54 & 13.5 & -22.8 & 0.57 \\
\hline 0.54 & 23.5 & -22.8 & 0.66 \\
\hline 0.54 & 0 & -29.8 & 0.00 \\
\hline 0.54 & 13.5 & -29.8 & 0.12 \\
\hline 0.54 & 23.5 & -29.8 & 0.00 \\
\hline & & & \\
\hline
\end{tabular}

Access to the mouth was at maximum values and actually increased as the arm moved past the mouth position $[-4,0,13.5]$ in Figure 4.7 toward the opposite side of the chair. The arm has very good access to the area directly in front of the chest of the operator. It is interesting to note that the Manus arm is capable of reaching across the centerline of the wheelchair to manipulate objects. In fact, in regions directly around the operator, the manipulability measure actually rises to a maximum value 6.75 " past the centerline of the wheelchair. 


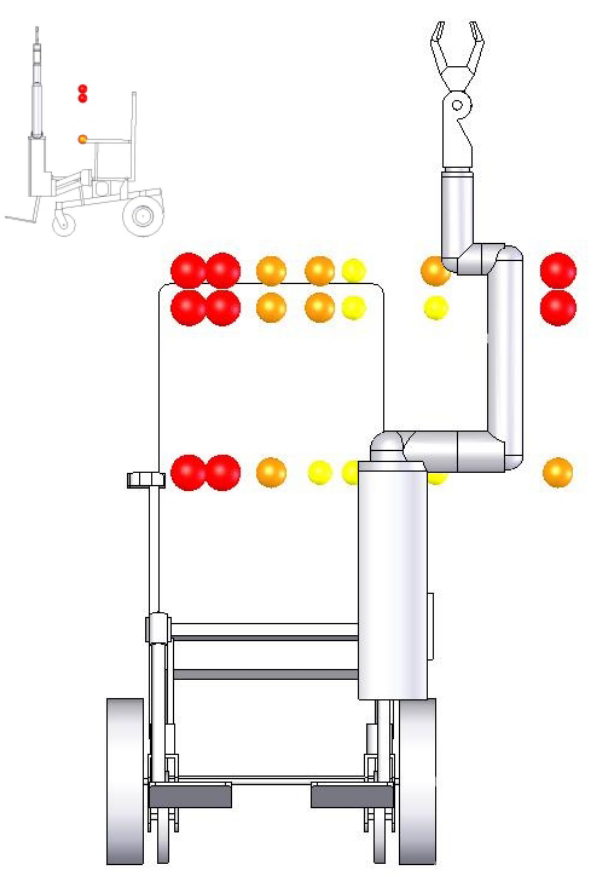

Figure 4.7:Manus y-z Plane @ x=0"
Table 4.6 : Data for Manus $\mathrm{x}=0$ ”

\begin{tabular}{|c|c|c|c|}
\hline $\mathbf{x}$ & $\mathbf{y}$ & $\mathbf{z}$ & $\mathbf{n}$ \\
\hline 0 & -6.75 & 16.5 & 0.94 \\
\hline 0 & -4 & 16.5 & 0.87 \\
\hline 0 & 0 & 16.5 & 0.75 \\
\hline 0 & 4 & 16.5 & 0.65 \\
\hline 0 & 6.75 & 16.5 & 0.60 \\
\hline 0 & 13.5 & 16.5 & 0.61 \\
\hline 0 & 23.5 & 16.5 & 0.85 \\
\hline 0 & -6.75 & 13.5 & 0.96 \\
\hline 0 & -4 & 13.5 & 0.86 \\
\hline 0 & 0 & 13.5 & 0.73 \\
\hline 0 & 4 & 13.5 & 0.62 \\
\hline 0 & 6.75 & 13.5 & 0.57 \\
\hline 0 & 13.5 & 13.5 & 0.58 \\
\hline 0 & 23.5 & 13.5 & 0.85 \\
\hline 0 & -6.75 & 0 & 0.94 \\
\hline 0 & -4 & 0 & 0.82 \\
\hline 0 & 0 & 0 & 0.65 \\
\hline 0 & 4 & 0 & 0.53 \\
\hline 0 & 6.75 & 0 & 0.48 \\
\hline 0 & 13.5 & 0 & 0.48 \\
\hline 0 & 23.5 & 0 & 0.80 \\
\hline
\end{tabular}

The maximum manipulability measure for the Manus manipulator was found at [$4,-6.75,13.5]$ in Figure 4.8. These values were determined through discrete analysis and are not necessarily a global maximum. The measure is increasing, moving from $\mathrm{x}=0$ through $x=-4$ to $x=-6.75$, where the last data point was on the $z=13.5$ " height.

The Manus has excellent manipulability measures at the vertical plane ( $\mathrm{x}=-4$ ”) at or near the height of the mouth $(\mathrm{z}=13.5$ " to 16.5 ") of the operator. The Manus provides excellent manipulator use when feeding and completing tasks that are at or about the mouth of the operator. 


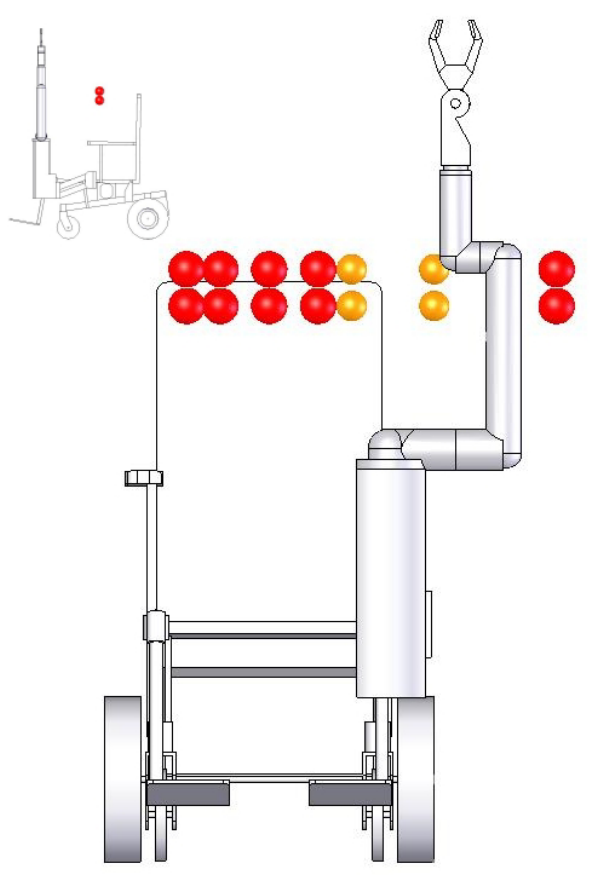

Table 4.7 : Data for Manus at $\mathrm{x}=-4$ "

\begin{tabular}{|c|c|c|c|}
\hline $\mathbf{x}$ & $\mathbf{y}$ & $\mathbf{z}$ & $\mathbf{n}$ \\
\hline-4 & -6.75 & 13.5 & 1.00 \\
\hline-4 & -4 & 13.5 & 0.95 \\
\hline-4 & 0 & 13.5 & 0.87 \\
\hline-4 & 4 & 13.5 & 0.80 \\
\hline-4 & 6.75 & 13.5 & 0.76 \\
\hline-4 & 13.5 & 13.5 & 0.76 \\
\hline-4 & 23.5 & 13.5 & 0.95 \\
\hline-4 & -6.75 & 16.5 & 0.95 \\
\hline-4 & -4 & 16.5 & 0.93 \\
\hline-4 & 0 & 16.5 & 0.86 \\
\hline-4 & 4 & 16.5 & 0.80 \\
\hline-4 & 6.75 & 16.5 & 0.77 \\
\hline-4 & 13.5 & 16.5 & 0.77 \\
\hline-4 & 23.5 & 16.5 & 0.92 \\
\hline
\end{tabular}

Figure 4.8 : Manus y-z Plane @ x = -4" 


\subsubsection{Horizontal Planes}

Approaching the analysis from horizontal slices better fit the requirements for designing a manipulator as an assistive device. Most objects rest on horizontal surfaces that have standard heights above ground level. An example is the standard desk height or the height of a light switch or a door handle.

These horizontal slices through the workspace also help to reflect the importance of wheelchair orientation for reaching a goal with a WMRA. A manipulator with very little access in one orientation but excellent access in another may require that the operator approach the goal with a different bearing to achieve the goal.

Starting at the top or highest defined plane, Figure 4.9 shows that for access to low kitchen cabinet shelves, the Manus has very good manipulability measures directly to the front and to the side of the chair.

The overall average of normalized manipulability is good at 0.53 . The closer the goal is to the first rotational axis, the lower the manipulability measure will be. This remains constant throughout all of the horizontal planes.

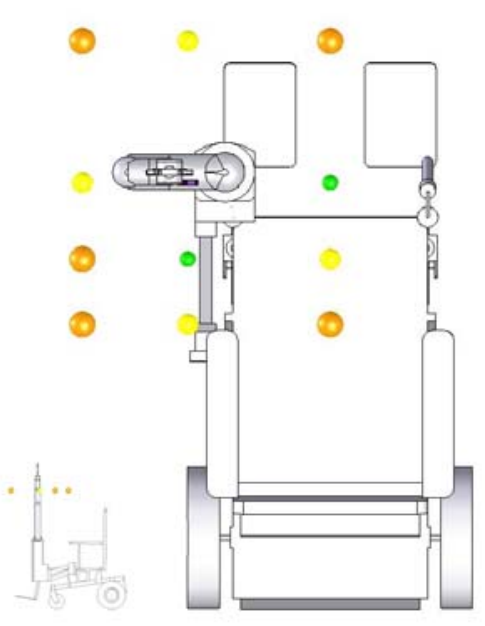

Figure 4.9 : Manus x-y Plane @ z=24.18”
Table 4.8 : Data for Manus at $\mathrm{z}=24.18 ”$

\begin{tabular}{|c|c|c|c|}
\hline $\mathbf{x}$ & $\mathbf{y}$ & $\mathbf{z}$ & $\mathbf{n}$ \\
\hline 27.54 & 0 & 24.18 & 0.64 \\
\hline 27.54 & 13.5 & 24.18 & 0.52 \\
\hline 27.54 & 23.5 & 24.18 & 0.72 \\
\hline 14.04 & 0 & 24.18 & 0.37 \\
\hline 14.04 & 13.5 & 24.18 & 0.00 \\
\hline 14.04 & 23.5 & 24.18 & 0.54 \\
\hline 6.75 & 0 & 24.18 & 0.52 \\
\hline 6.75 & 13.5 & 24.18 & 0.32 \\
\hline 6.75 & 23.5 & 24.18 & 0.64 \\
\hline 0.54 & 0 & 24.18 & 0.70 \\
\hline 0.54 & 13.5 & 24.18 & 0.60 \\
\hline 0.54 & 23.5 & 24.18 & 0.75 \\
\hline
\end{tabular}

In Figure 4.10 the horizontal plane of a light switch $(\mathrm{z}=18.2$ ") can be seen. 
When attempting to operate a light switch, the optimum approach is from the side because the $n$ value directly to the side of the operator is excellent $(0.83)$. The manipulability measure is still very good in the far front of the operator and to the far left corner of the workspace.

The average $n$-value for this plane is good at 0.53 . Access to the inside the perimeter of the workspace are limited.

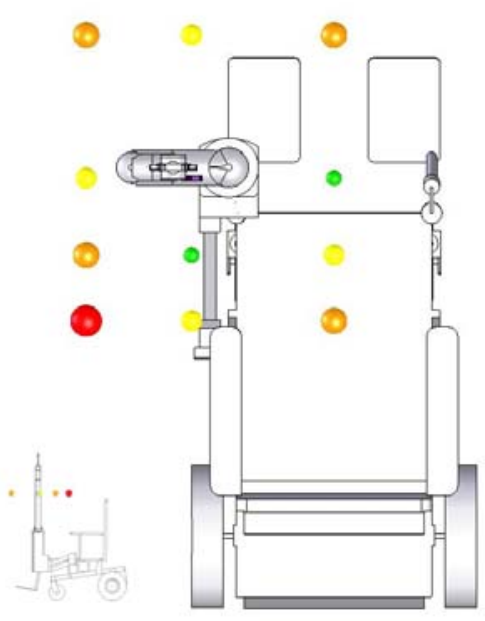

Figure 4.10 : Manus x-y Plane @ z=18.2”
Table 4.9 : Data for Manus at $\mathrm{z}=18.2$ "

\begin{tabular}{|c|c|c|c|}
\hline $\mathbf{x}$ & $\mathbf{y}$ & $\mathbf{z}$ & $\mathbf{n}$ \\
\hline 27.54 & 0 & 18.2 & 0.65 \\
\hline 27.54 & 13.5 & 18.2 & 0.49 \\
\hline 27.54 & 23.5 & 18.2 & 0.77 \\
\hline 14.04 & 0 & 18.2 & 0.34 \\
\hline 14.04 & 13.5 & 18.2 & 0.00 \\
\hline 14.04 & 23.5 & 18.2 & 0.52 \\
\hline 6.75 & 0 & 18.2 & 0.49 \\
\hline 6.75 & 13.5 & 18.2 & 0.28 \\
\hline 6.75 & 23.5 & 18.2 & 0.64 \\
\hline 0.54 & 0 & 18.2 & 0.73 \\
\hline 0.54 & 13.5 & 18.2 & 0.59 \\
\hline 0.54 & 23.5 & 18.2 & 0.83 \\
\hline
\end{tabular}

At the kitchen countertop level, Figure 4.11, the manipulator's effectiveness drops significantly. This is due in part to the plane being observed having very little vertical separation from the plane that the first link rotates along. This would make the manipulability measure reach low values in the horizontal plane of $z=1.74$ ". Because the planes of the countertop $(z=6.16$ " Figure 4.11) and desktop / door handle $(z=-0.8$ " Figure 4.12) are close to this plane, they have very similar values for the manipulability measure.

The average $n$-value for the plane is good at 0.42 . The highest $n$-value on this plane is to the left of the operator. Accessibility of objects within the workspace are best when attempted close to the operator, such as at $\mathrm{x}=0.54$ compared to any other yz-plane. 


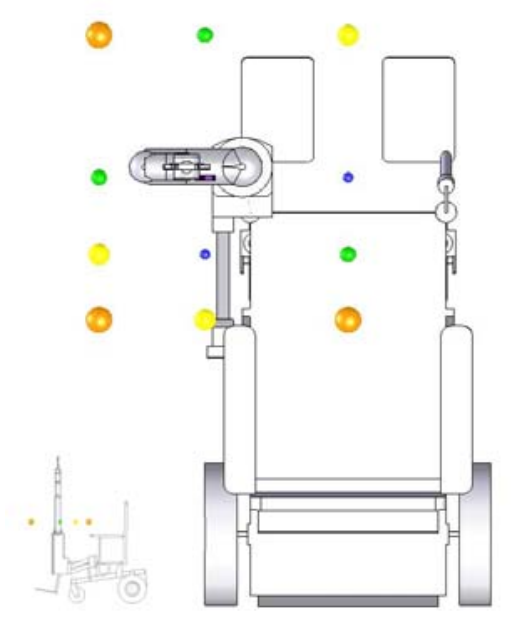

Figure 4.11 : Manus x-y Plane @ $\mathrm{z}=6.18 ”$
Table 4.10 : Data for Manus at $\mathrm{z}=6.18$ ”

\begin{tabular}{|c|c|c|c|}
\hline $\mathbf{x}$ & $\mathbf{y}$ & $\mathbf{z}$ & $\mathbf{n}$ \\
\hline 27.54 & 0 & 6.18 & 0.53 \\
\hline 27.54 & 13.5 & 6.18 & 0.35 \\
\hline 27.54 & 23.5 & 6.18 & 0.69 \\
\hline 14.04 & 0 & 6.18 & 0.19 \\
\hline 14.04 & 13.5 & 6.18 & 0.00 \\
\hline 14.04 & 23.5 & 6.18 & 0.38 \\
\hline 6.75 & 0 & 6.18 & 0.35 \\
\hline 6.75 & 13.5 & 6.18 & 0.14 \\
\hline 6.75 & 23.5 & 6.18 & 0.51 \\
\hline 0.54 & 0 & 6.18 & 0.63 \\
\hline 0.54 & 13.5 & 6.18 & 0.46 \\
\hline 0.54 & 23.5 & 6.18 & 0.78 \\
\hline
\end{tabular}

The Manus arm manipulability at the height of a table or door knob is shown in Figure 4.12. At this plane the interior regions of the plane have very limited accessibility. At the outer corners of the workspace the arm has good or better access to objects with the maximum manipulability, for this plane, to the left of the operator.

The average n-value for the plane is 0.41 and is the poorest average plane value with the exception of lowest plane. This plane is marginally good on the qualitative scale. 


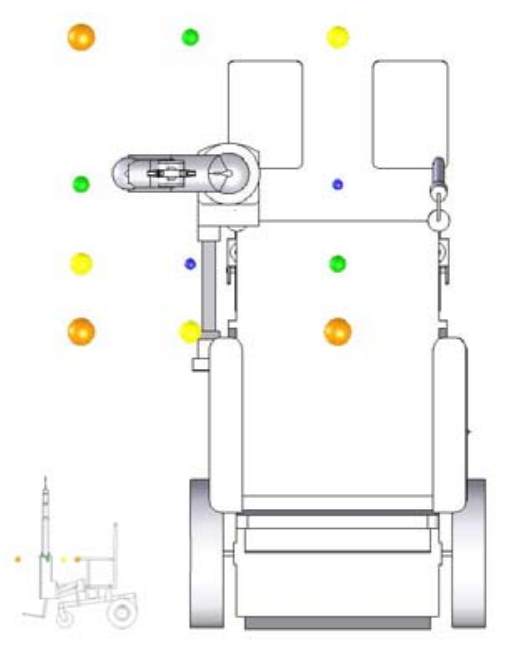

Figure 4.12 : Manus x-y Plane @ $\mathrm{z}=-0.8$ ”
Table 4.11 : Data for Manus at $\mathrm{z}=-0.8$ "

\begin{tabular}{|c|c|c|c|}
\hline $\mathbf{x}$ & $\mathbf{y}$ & $\mathbf{z}$ & $\mathbf{n}$ \\
\hline 27.54 & 0 & -0.8 & 0.52 \\
\hline 27.54 & 13.5 & -0.8 & 0.33 \\
\hline 27.54 & 23.5 & -0.8 & 0.68 \\
\hline 14.04 & 0 & -0.8 & 0.18 \\
\hline 14.04 & 13.5 & -0.8 & 0.00 \\
\hline 14.04 & 23.5 & -0.8 & 0.37 \\
\hline 6.75 & 0 & -0.8 & 0.33 \\
\hline 6.75 & 13.5 & -0.8 & 0.13 \\
\hline 6.75 & 23.5 & -0.8 & 0.51 \\
\hline 0.54 & 0 & -0.8 & 0.62 \\
\hline 0.54 & 13.5 & -0.8 & 0.45 \\
\hline 0.54 & 23.5 & -0.8 & 0.77 \\
\hline
\end{tabular}

Manus arm at the height of a coffee table is shown in Figure 4.13. Access to an object to the side of the operator is good and has its highest value of $n(0.79)$ left of the operator's hand $(0.54,23.5,-5.82)$.

The average $n$-value for this plane is good at 0.44 . The arm has lower accessibility near the centerline of the wheelchair $(y=0)$ compared with the outer edge of the workspace $(\mathrm{y}=23.5)$. 


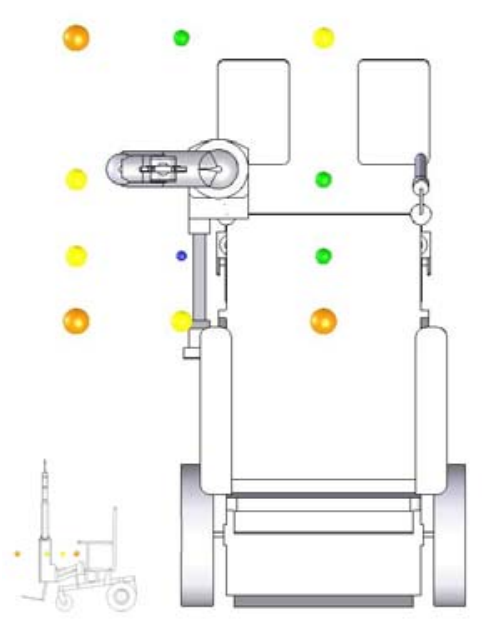

Figure 4.13 : Manus x-y Plane @ z= -5.82”
Table 4.12: Data for Manus at $\mathrm{z}=-5.82$ ”

\begin{tabular}{|c|c|c|c|}
\hline $\mathbf{x}$ & $\mathbf{y}$ & $\mathbf{z}$ & $\mathbf{n}$ \\
\hline 27.54 & 0 & -5.82 & 0.56 \\
\hline 27.54 & 13.5 & -5.82 & 0.38 \\
\hline 27.54 & 23.5 & -5.82 & 0.71 \\
\hline 14.04 & 0 & -5.82 & 0.23 \\
\hline 14.04 & 13.5 & -5.82 & 0.00 \\
\hline 14.04 & 23.5 & -5.82 & 0.41 \\
\hline 6.75 & 0 & -5.82 & 0.37 \\
\hline 6.75 & 13.5 & -5.82 & 0.18 \\
\hline 6.75 & 23.5 & -5.82 & 0.55 \\
\hline 0.54 & 0 & -5.82 & 0.66 \\
\hline 0.54 & 13.5 & -5.82 & 0.49 \\
\hline 0.54 & 23.5 & -5.82 & 0.79 \\
\hline
\end{tabular}

Closer to the ground, the measure begins to rise and access to an electric socket ( $\mathrm{z}$ $=-13.8$ " Figure 4.14) is excellent to the side of the chair and still very high in front of the wheelchair. It can be noted here that this is the lowest plane that still has good or better qualitative rating.

The average $n$-value for this plane is good at 0.52 but the range of manipulability measures within this plane is larger than other planes. The lowest reachable $n$-value is 0.27 and the highest is 0.83 . 


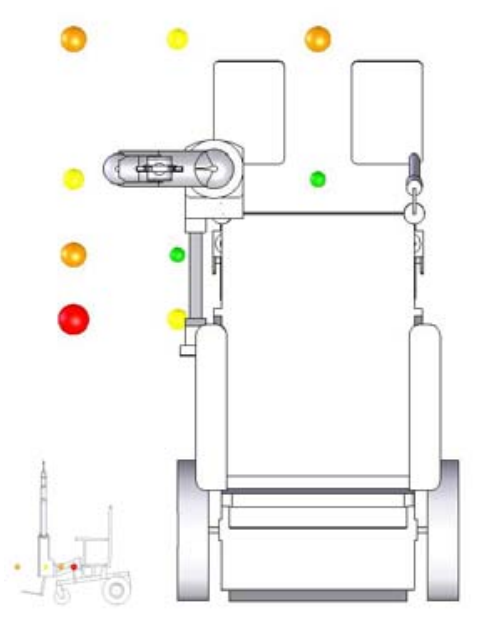

Table 4.13 : Data for Manus at $\mathrm{z}=-13.8$ "

\begin{tabular}{|c|c|c|c|}
\hline $\mathbf{x}$ & $\mathbf{y}$ & $\mathbf{z}$ & $\mathbf{n}$ \\
\hline 27.54 & 0 & -13.8 & 0.64 \\
\hline 27.54 & 13.5 & -13.8 & 0.48 \\
\hline 27.54 & 23.5 & -13.8 & 0.77 \\
\hline 14.04 & 0 & -13.8 & 0.33 \\
\hline 14.04 & 13.5 & -13.8 & 0.00 \\
\hline 14.04 & 23.5 & -13.8 & 0.51 \\
\hline 6.75 & 0 & -13.8 & 0.47 \\
\hline 6.75 & 13.5 & -13.8 & 0.27 \\
\hline 6.75 & 23.5 & -13.8 & 0.64 \\
\hline 0.54 & 0 & -13.8 & 0.72 \\
\hline 0.54 & 13.5 & -13.8 & 0.58 \\
\hline 0.54 & 23.5 & -13.8 & 0.83 \\
\hline
\end{tabular}

Figure 4.14 : Manus x-y Plane @ z = -13.8”

For tall objects on the ground, accessibility is very good directly to the front of and to the sides of the wheelchair. Reaching this plane is best from the side of the wheelchair with the average value along the right side of the plane being 0.62 . Overall the plane has a good average $n$-value of 0.50 . 


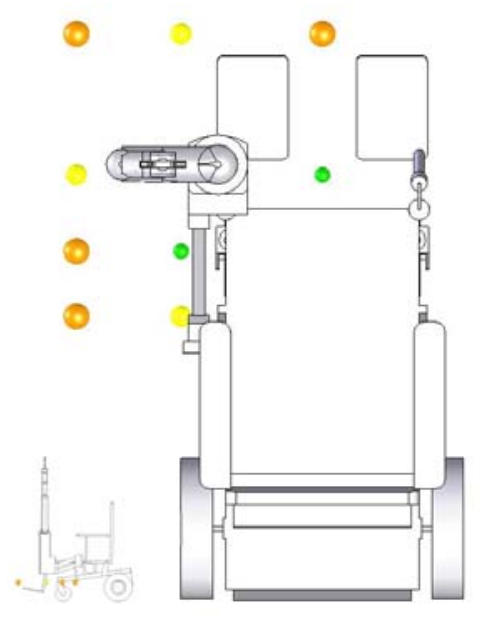

Figure 4.15 : Manus x-y Plane @ z= - 22.8”
Table 4.14 : Data for Manus at $\mathrm{z}=-22.8$ "

\begin{tabular}{|c|c|c|c|}
\hline $\mathbf{x}$ & $\mathbf{y}$ & $\mathbf{z}$ & $\mathbf{n}$ \\
\hline 27.54 & 0 & -22.8 & 0.61 \\
\hline 27.54 & 13.5 & -22.8 & 0.50 \\
\hline 27.54 & 23.5 & -22.8 & 0.66 \\
\hline 14.04 & 0 & -22.8 & 0.37 \\
\hline 14.04 & 13.5 & -22.8 & 0.00 \\
\hline 14.04 & 23.5 & -22.8 & 0.53 \\
\hline 6.75 & 0 & -22.8 & 0.50 \\
\hline 6.75 & 13.5 & -22.8 & 0.32 \\
\hline 6.75 & 23.5 & -22.8 & 0.61 \\
\hline 0.54 & 0 & -22.8 & 0.65 \\
\hline 0.54 & 13.5 & -22.8 & 0.57 \\
\hline 0.54 & 23.5 & -22.8 & 0.66 \\
\hline
\end{tabular}

Objects low to the ground, shown in Figure 4.16, are at the lower limit of the reach of the Manus manipulator. The arm is not capable of reaching objects in the far corners of the workspace on the horizontal plane at $\mathrm{z}=-29.8$ ". The average $n$-value for all the points on the plane is 0.10 . This means that the manipulator has very limited access to the plane.

It is interesting to note that regions where the arm traditionally has lower manipulability measures are the areas that have the highest measures at $z=-29.8$ '. These zones are the vertical lines that share the points $(27.54,13.5, \mathrm{z})$ and $(14.04,23.5, \mathrm{z})$. 


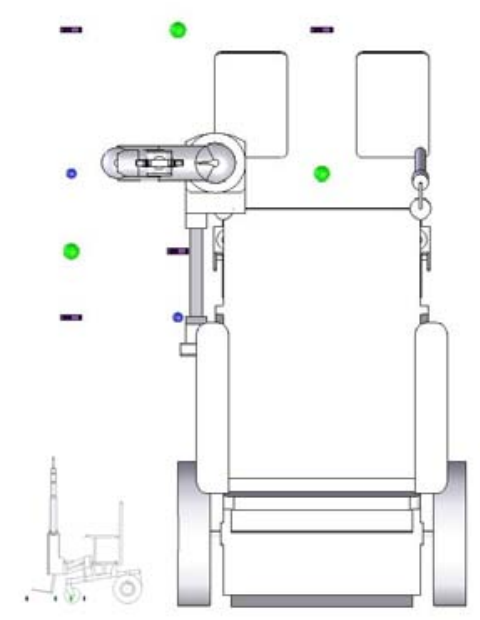

Figure 4.16 : Manus x-y Plane @ z= - 29.8”
Table 4.15 : Data for Manus at $\mathrm{z}=-29.8$ "

\begin{tabular}{|c|c|c|c|}
\hline $\mathbf{x}$ & $\mathbf{y}$ & $\mathbf{z}$ & $\mathbf{n}$ \\
\hline 27.54 & 0 & -29.8 & 0.00 \\
\hline 27.54 & 13.5 & -29.8 & 0.22 \\
\hline 27.54 & 23.5 & -29.8 & 0.00 \\
\hline 14.04 & 0 & -29.8 & 0.23 \\
\hline 14.04 & 13.5 & -29.8 & 0.00 \\
\hline 14.04 & 23.5 & -29.8 & 0.20 \\
\hline 6.75 & 0 & -29.8 & 0.25 \\
\hline 6.75 & 13.5 & -29.8 & 0.21 \\
\hline 6.75 & 23.5 & -29.8 & 0.00 \\
\hline 0.54 & 0 & -29.8 & 0.00 \\
\hline 0.54 & 13.5 & -29.8 & 0.12 \\
\hline 0.54 & 23.5 & -29.8 & 0.00 \\
\hline
\end{tabular}

A summary of effectiveness in reaching areas common to activities of daily living (ADL) is shown in Table 4.16. The qualitative assessment is based on the average of the normalized manipulability measure of all possible wheelchair orientations possible to accomplish the task. Six possible qualitative assessments could be given for each task. These qualitative assessments were first shown in Figure 4.2 and are: excellent, very good, good, limited, very limited, and undefined or unreachable.

For example, an object can be picked off the ground from in front of the wheelchair as well as along the side. The average of all the recorded values for the normalized manipulability measure $(n)$ along the entire perimeter of the wheelchair at the plane of the specific activity of daily living is shown in the second column of Table 4.16.

Each row involved only one horizontal plane except for picking up objects on the ground and access to mouth. For the ADL "Pick-up off Ground" the average value for the perimeter of the chair was taken for the two lowest planes $(z=-22.8$ " and $z=-29.8$ "). The ADL "Access to mouth" took the average of three points on two vertical planes $\mathrm{x}=$ 0 " and $\mathrm{x}=-4$ ". Because head position may not be perfectly in the centerline of the wheelchair points, $y=4$ ", 0 ", -4 " were averaged at both head heights $(z=13.5$ " and $z=$ $\left.16.5^{\prime \prime}\right)$. 
The method used for determining the qualitative efficacy of a manipulator is not orientation-specific. In every instance of ADL analysis, there was a wheelchair orientation that would provide a higher normalized manipulability measure than the value listed in Table 4.16. If the operator is given the ability to maneuver the wheelchair into a specific position for the job, the manipulator would be able to have a greater manipulability measure for that goal.

\begin{tabular}{|c|c|c|}
\hline Pick-up off ground & 0.33 & Limited \\
\hline Coffee table & 0.57 & Good \\
\hline Door knob & 0.53 & Good \\
\hline Kitchen countertop & 0.54 & Good \\
\hline Light switch & 0.65 & Very Good \\
\hline Low kitchen shelf & 0.64 & Very Good \\
\hline Reach into lap & 0.57 & Good \\
\hline Access to mouth & 0.81 & Excellent \\
\hline
\end{tabular}

The Manus manipulator provided excellent (0.81) access to the mouth of the operator. The ability to do tasks above the kitchen countertop height such as reach a light switch and reach a low kitchen shelf was very good. Reaching from the side of the operator would yield the highest manipulability measures from the arm.

Access to a coffee table and the operator's lap were close to very good and had a qualitative rating of good (0.57). The ability to reach a doorknob and a kitchen countertop were both good ( 0.53 and 0.54 respectively).

Finally, the lowest value for the activities listed in Table 4.16 was reaching the floor. Access to the floor was limited with a value of 0.33 . 


\subsection{Evaluation of the Raptor}

The next system to be analyzed is the Raptor. Because all power wheelchairs are constructed differently, the mount used in this application is specific to the chair that was available. Of the two manipulators that were analyzed, only the Raptor was available for direct measurements of the complete system. The power wheelchair that the solid model was created from was a Storm Series "Arrow".

The Raptor motor fit under the chair but there was difficulty finding a satisfactory mounting position with the factory-provided mounts. To solve the problem, replacement mounting brackets were fabricated from aluminum in order to achieve a level of structural integrity that showed the overall stiffness of the robotic arm without the mounting adding to positioning error. With the Raptor manipulator mounted securely, a significant amount (1" - 2") of play could be felt at the end effector. 


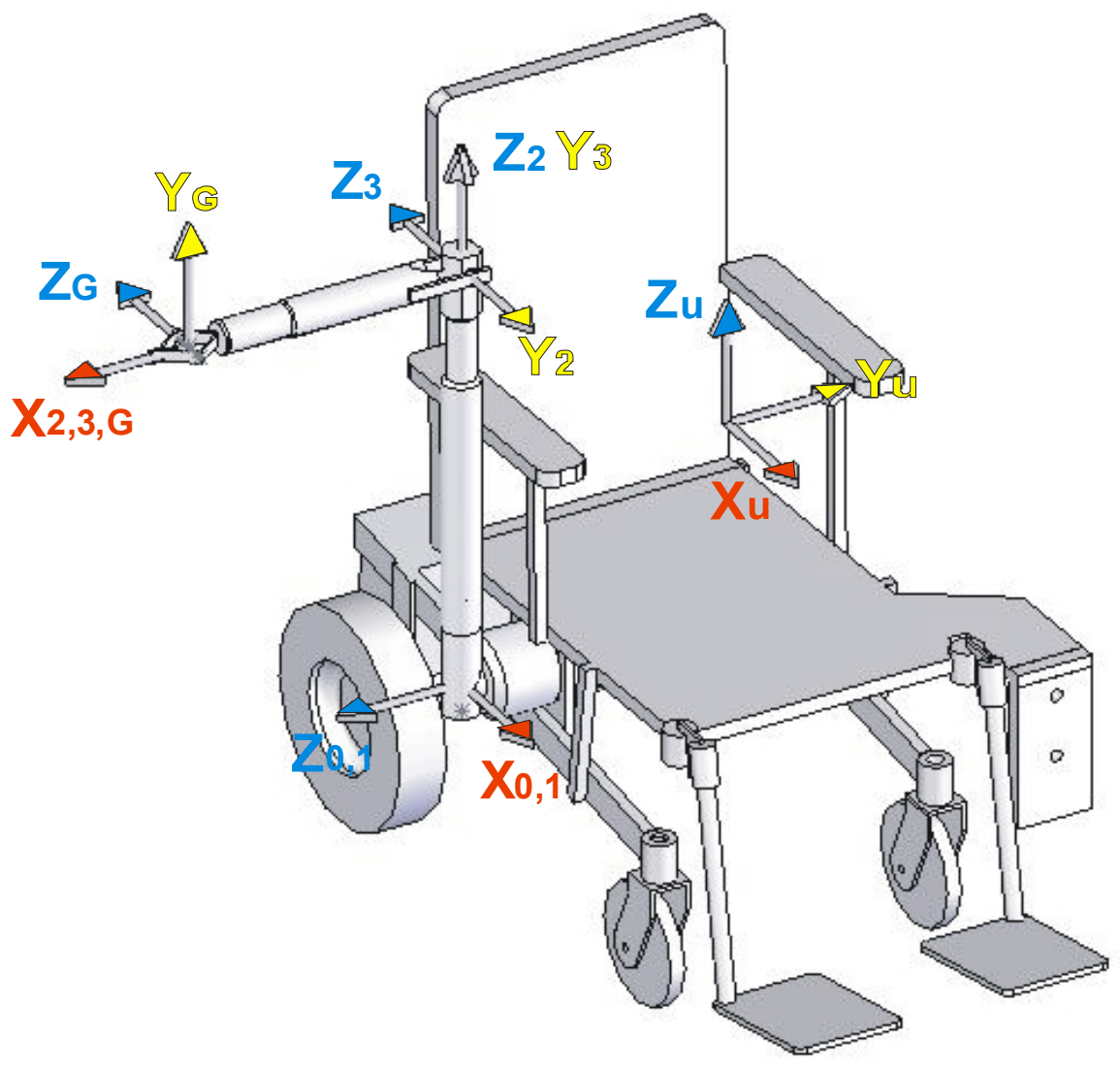

Figure 4.17 : Raptor Reference Frames

Figure 4.17 represents the Raptor mounted to a generic power wheelchair along with the corresponding frames of reference (frames 0 to $G$ ).

Table 4.17 shows the D-H parameter table for the Raptor with 3DOF. This table of D-H parameters, along with Figure 4.17 and Equation 3.2, are used to create the transformation matrix which relates the link $i$ with previous link $i-1$. These individual transformation matrices for each link are shown after the table of parameters. There is also a row for the fourth link. This link connects the gripper to the third joint. It is purely a translational relation, so the coordinate system for the third joint and the gripper have the same orientation. 
Table 4.17 : Raptor D-H Parameters

\begin{tabular}{|l|l|l|l|l|}
\hline $\mathrm{i}$ & $\alpha_{\mathrm{i}-1}$ & $\mathrm{a}_{\mathrm{i}-1}$ & $\mathrm{~d}_{\mathrm{i}}$ & $\theta_{\mathrm{i}}$ \\
\hline 1 & $0^{\circ}$ & 0 & 5 & $\theta_{1}$ \\
\hline 2 & $90^{\circ}$ & 0 & 27 & $\theta_{2}$ \\
\hline 3 & $-90^{\circ}$ & 0 & 0 & $\theta_{3}$ \\
\hline 4 & $0^{\circ}$ & 15.75 & 0 & 0 \\
\hline
\end{tabular}

Inserting the transformation matrices from the DH-Parameter into the table shown in Table 4.17 provides the transformation matrix that relates frame 1 with respect to frame 0 (Equation 4.10).

$$
\operatorname{T1} \operatorname{wrt} 0:=\left(\begin{array}{cccc}
\cos (\theta 1) & -\sin (\theta 1) & 0 & 0 \\
\sin (\theta 1) & \cos (\theta 1) & 0 & 0 \\
0 & 0 & 1 & 0 \\
0 & 0 & 0 & 1
\end{array}\right)
$$

The transformation matrix which relates frame 2 with respect to frame 1 is shown in Equation 4.11.

$$
\operatorname{T} 2 \operatorname{wrt} 1:=\left(\begin{array}{cccc}
\sin (\theta 2) & \cos (\theta 2) & 0 & 0 \\
0 & 0 & 1 & 27 \\
\cos (\theta 2) & -\sin (\theta 2) & 0 & 0 \\
0 & 0 & 0 & 1
\end{array}\right)
$$

The transformation matrix which relates frame 3 with respect to frame 2 is shown in Equation 3.9 the Equation below.

$$
\text { T3wrt2 : }=\left(\begin{array}{cccc}
\cos (\theta 3) & -\sin (\theta 3) & 0 & 0 \\
0 & 0 & -1 & 0 \\
\sin (\theta 3) & \cos (\theta 3) & 0 & 0 \\
0 & 0 & 0 & 1
\end{array}\right)
$$


The transformation matrix which relates frame $\mathrm{G}$, or the frame of the gripper, with respect to frame 3 is shown in Equation 4.13.

$$
\text { TGwrt3:=( } \left.\begin{array}{cccc}
1 & 0 & 0 & 18.38 \\
0 & 1 & 0 & 0 \\
0 & 0 & 1 & 0 \\
0 & 0 & 0 & 1
\end{array}\right)
$$

It is necessary to work with a frame that has correlation to the user, therefore a user frame must be created. Equation 4.14 shows the transformation matrix which defines the translational relationship between frame 0 with respect to the user frame.

$$
\text { T0wrtU }:=\left(\begin{array}{cccc}
1 & 0 & 0 & -6.30 \\
0 & 0 & -1 & -13.46 \\
0 & 1 & 0 & -16.16 \\
0 & 0 & 0 & 1
\end{array}\right)
$$

Concatenation of the transformation matrices is shown in Equation 4.15, to give the transform relating the end gripper position with respect to the user frame. This will be the complete transformation matrix.

$$
\text { T0wrtU*T1wrt0*T2wrt1*T3wrt2*TGwrt3 = TGwrtU Equation } 4.15
$$

The final transformational matrix is shown in Equation 4.16 the figure below. $\left(\begin{array}{cccc}\cos (\theta 1) \cdot \sin (\theta 2) \cdot \cos (\theta 3)-\sin (\theta 1) \cdot \sin (\theta 3) & -\cos (\theta 1) \cdot \sin (\theta 2) \cdot \sin (\theta 3)-\sin (\theta 1) \cdot \cos (\theta 3) & -\cos (\theta 1) \cdot \cos (\theta 2) & 18.38 \cos (\theta 1) \cdot \sin (\theta 2) \cdot \cos (\theta 3)-18.38 \sin (\theta 1) \cdot \sin (\theta 3)+6.30-27 \cdot \sin (\theta 1) \\ -\cos (\theta 2) \cdot \cos (\theta 3) & \cos (\theta 2) \cdot \sin (\theta 3) & -\sin (\theta 2) & -18.38 \cos (\theta 2) \cdot \cos (\theta 3)-13.46 \\ \sin (\theta 1) \cdot \sin (\theta 2) \cdot \cos (\theta 3)+\cos (\theta 1) \cdot \sin (\theta 3) & -\sin (\theta 1) \cdot \sin (\theta 2) \cdot \sin (\theta 3)+\cos (\theta 1) \cdot \cos (\theta 3) & -\sin (\theta 1) \cdot \cos (\theta 2) & 18.38 \sin (\theta 1) \cdot \sin (\theta 2) \cdot \cos (\theta 3)+18.38 \cos (\theta 1) \cdot \sin (\theta 3)-16.16+27 \cdot \cos (\theta 1)\end{array}\right)$

The position vector, a 3x1 matrix, is the first three rows of the final column of the final transformational matrix and is shown in the figure below. This matrix is also known 
as the forward kinematic matrix. When the three joint angles are input, the result is the position vector (Equation 4.17) of the gripper with respect to the user frame.

$$
\left(\begin{array}{rr}
18.38 \cos (\theta 1) \cdot \sin (\theta 2) \cdot \cos (\theta 3)-18.38 \sin (\theta 1) \cdot \sin (\theta 3)-6.30-27 \cdot \sin (\theta 1) & \\
-18.38 \cos (\theta 2) \cdot \cos (\theta 3)-13.46 & \text { Equation } 4.17 \\
18.38 \sin (\theta 1) \cdot \sin (\theta 2) \cdot \cos (\theta 3)+18.38 \cos (\theta 1) \cdot \sin (\theta 3)-16.16+27 \cdot \cos (\theta 1) &
\end{array}\right.
$$

From the forward kinematic matrix we can compute the Jacobian Matrix - the partial derivatives of the positional matrix as shown Equation 4.18. Due to the size of the Jacobian, the matrix will be displayed one column at a time.

Column 1:

$-18.38^{*} \sin (\mathrm{t} 1)^{*} \sin (\mathrm{t} 2)^{*} \cos (\mathrm{t} 3)-18.38(\mathrm{t} 1)^{*} \sin (\mathrm{t} 3)-27^{*} \cos (\mathrm{t} 1)$

0

$18.38^{*} \cos (\mathrm{t} 1)^{*} \sin (\mathrm{t} 2)^{*} \cos (\mathrm{t} 3)-18.38^{*} \sin (\mathrm{t} 1)^{*} \sin (\mathrm{t} 3)-27^{*} \sin (\mathrm{t} 1)$

Equation 4.18

Column 2:

$$
\begin{gathered}
18.38^{*} \cos (\mathrm{t} 1)^{*} \cos (\mathrm{t} 2)^{*} \cos (\mathrm{t} 3) \\
18.38^{*} \sin (\mathrm{t} 2)^{*} \cos (\mathrm{t} 3) \\
18.38^{*} \sin (\mathrm{t} 1)^{*} \cos (\mathrm{t} 2)^{*} \cos (\mathrm{t} 3)
\end{gathered}
$$

Column 3:

$$
\begin{gathered}
-18.38^{*} \cos (\mathrm{t} 1)^{*} \sin (\mathrm{t} 2)^{*} \sin (\mathrm{t} 3)-18.38^{*} \sin (\mathrm{t} 1)^{*} \cos (\mathrm{t} 3) \\
18.38^{*} \cos (\mathrm{t} 2)^{*} \sin (\mathrm{t} 3) \\
-18.38^{*} \sin (\mathrm{t} 1)^{*} \sin (\mathrm{t} 2)^{*} \sin (\mathrm{t} 3)+18.38^{*} \cos (\mathrm{t} 1)^{*} \cos (\mathrm{t} 3)
\end{gathered}
$$

\subsubsection{Vertical Planes}

The following figures show the manipulability measures for the Raptor arm as mounted on the Arrow Storm Series power wheelchair.

After all data points were collected, the maximum manipulability measure for the Raptor was found to be 9121.0 at the point $[-4,-13.5,16.5]$.

Comments regarding the data: 
As shown in Figure 4.18, the Raptor is incapable of reaching objects on a high shelf. At this distance of 27.54" in front of the user frame, operating a light switch is only possible when directly in line with the plane of the first link's rotation (xz plane where y $=-13.5 ")$, which also is the plane that has maximum manipulability measure values.

The Raptor has very good manipulability from the ground to the height of a low coffee table $\left(\mathrm{z}=-5.82^{\prime \prime}\right)$ and still has good manipulability at the height of a standard table $(\mathrm{z}=-0.8$ ').

At this distance from the user frame, the arm is not capable of reaching objects on a low shelf $(z=24.18)$. A light switch can only be manipulated directly along a lateral line of $y=-13.5 "$ 
Table 4.18 : Data for Raptor at $\mathrm{x}=27.54$ "

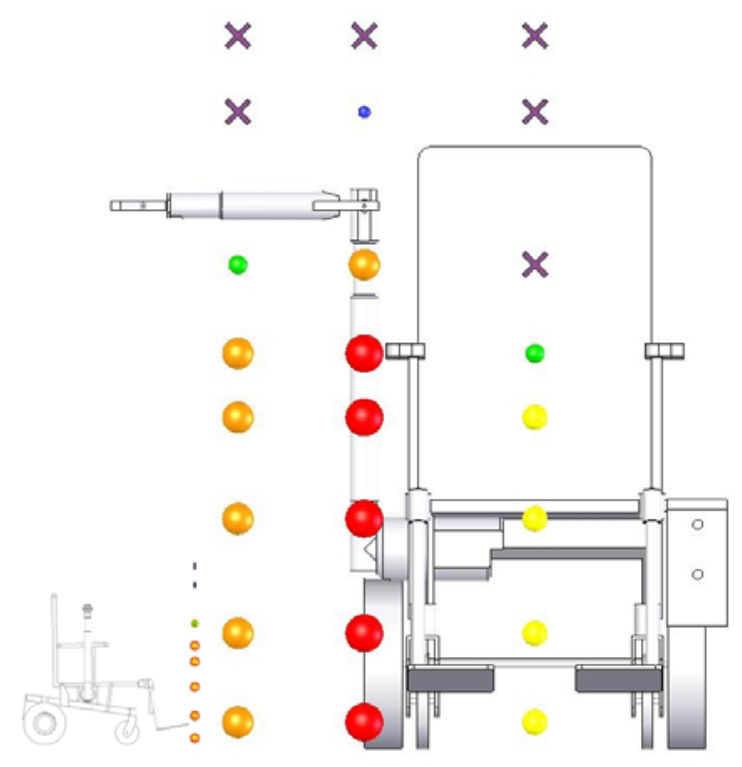

\begin{tabular}{|c|c|c|c|}
\hline $\mathrm{x}$ & $\mathrm{y}$ & $\mathrm{z}$ & $\mathrm{n}$ \\
\hline 27.54 & 0 & -29.8 & 0.46 \\
\hline 27.54 & -13.5 & -29.8 & 0.93 \\
\hline 27.54 & -23.5 & -29.8 & 0.70 \\
\hline 27.54 & 0 & -22.8 & 0.58 \\
\hline 27.54 & -13.5 & -22.8 & 0.98 \\
\hline 27.54 & -23.5 & -22.8 & 0.79 \\
\hline 27.54 & 0 & -13.8 & 0.60 \\
\hline 27.54 & -13.5 & -13.8 & 0.99 \\
\hline 27.54 & -23.5 & -13.8 & 0.80 \\
\hline 27.54 & 0 & -5.82 & 0.53 \\
\hline 27.54 & -13.5 & -5.82 & 0.97 \\
\hline 27.54 & -23.5 & -5.82 & 0.76 \\
\hline 27.54 & 0 & -0.8 & 0.40 \\
\hline 27.54 & -13.5 & -0.8 & 0.90 \\
\hline 27.54 & -23.5 & -0.8 & 0.66 \\
\hline 27.54 & 0 & 6.18 & 0.00 \\
\hline 27.54 & -13.5 & 6.18 & 0.66 \\
\hline 27.54 & -23.5 & 6.18 & 0.35 \\
\hline 27.54 & 0 & 18.2 & 0.00 \\
\hline 27.54 & -13.5 & 18.2 & 0.24 \\
\hline 27.54 & -23.5 & 18.2 & 0.00 \\
\hline 27.54 & 0 & 24.18 & 0.00 \\
\hline 27.54 & -13.5 & 24.18 & 0.00 \\
\hline 27.54 & -23.5 & 24.18 & 0.00 \\
\hline & & & \\
\hline
\end{tabular}

Figure 4.18 : Raptor y-z Plane @ $\mathrm{x}=27.54$ "

From Figure 4.19 it can be seen that the ability of the Raptor manipulator to reach low kitchen cabinet shelves is very limited.

At this distance $(\mathrm{x}=14.04$ ") the operator would have to position his/her feet approximately 20 " under a countertop. This is not possible with some kitchen designs ${ }^{2}$. At this distance, accessibility of objects on tables and countertops is excellent from the front. Access to all levels except for the low shelf is very good from the side. 
Table 4.19 : Data for Raptor at $\mathrm{x}=14.04 ”$

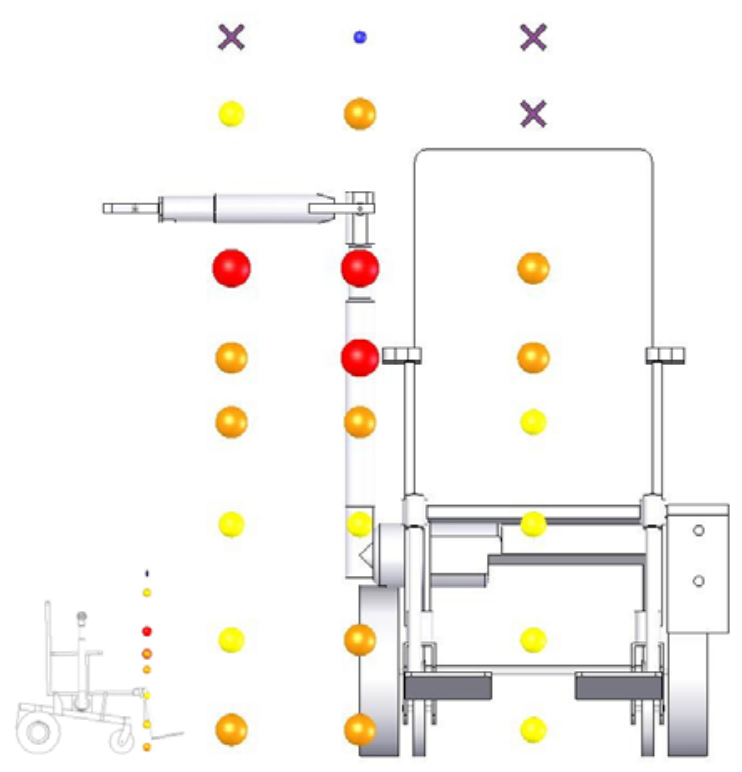

\begin{tabular}{|c|c|c|c|}
\hline $\mathrm{x}$ & $\mathrm{y}$ & $\mathrm{z}$ & $\mathrm{n}$ \\
\hline 14.04 & 0 & -29.8 & 0.59 \\
\hline 14.04 & -13.5 & -29.8 & 0.78 \\
\hline 14.04 & -23.5 & -29.8 & 0.70 \\
\hline 14.04 & 0 & -22.8 & 0.48 \\
\hline 14.04 & -13.5 & -22.8 & 0.62 \\
\hline 14.04 & -23.5 & -22.8 & 0.57 \\
\hline 14.04 & 0 & -13.8 & 0.44 \\
\hline 14.04 & -13.5 & -13.8 & 0.57 \\
\hline 14.04 & -23.5 & -13.8 & 0.53 \\
\hline 14.04 & 0 & -5.82 & 0.53 \\
\hline 14.04 & -13.5 & -5.82 & 0.70 \\
\hline 14.04 & -23.5 & -5.82 & 0.63 \\
\hline 14.04 & 0 & -0.8 & 0.62 \\
\hline 14.04 & -13.5 & -0.8 & 0.82 \\
\hline 14.04 & -23.5 & -0.8 & 0.73 \\
\hline 14.04 & 0 & 6.18 & 0.68 \\
\hline 14.04 & -13.5 & 6.18 & 0.98 \\
\hline 14.04 & -23.5 & 6.18 & 0.83 \\
\hline 14.04 & 0 & 18.2 & 0.00 \\
\hline 14.04 & -13.5 & 18.2 & 0.72 \\
\hline 14.04 & -23.5 & 18.2 & 0.42 \\
\hline 14.04 & 0 & 24.18 & 0.00 \\
\hline 14.04 & -13.5 & 24.18 & 0.04 \\
\hline 14.04 & -23.5 & 24.18 & 0.00 \\
\hline & & & \\
\hline
\end{tabular}

Figure 4.19 : Raptor y-z Plane @ x = 14.04”

At the yz-plane where $\mathrm{x}=6.75$ ", shown in Figure 4.20, the Raptor is finally able to reach objects on low shelves with more than a minimal value. The ability to reach objects to the side of the chair begins to drop off, although it still can reach the ground with a good manipulability measure. Access to the side at this plane is good but all eight horizontal planes can be reached at this distance from the operator. This is the only vertical plane that offers this ability to reach all horizontal planes with the Raptor. 
Table 4.20 : Data for Raptor at $\mathrm{x}=6.75$ "

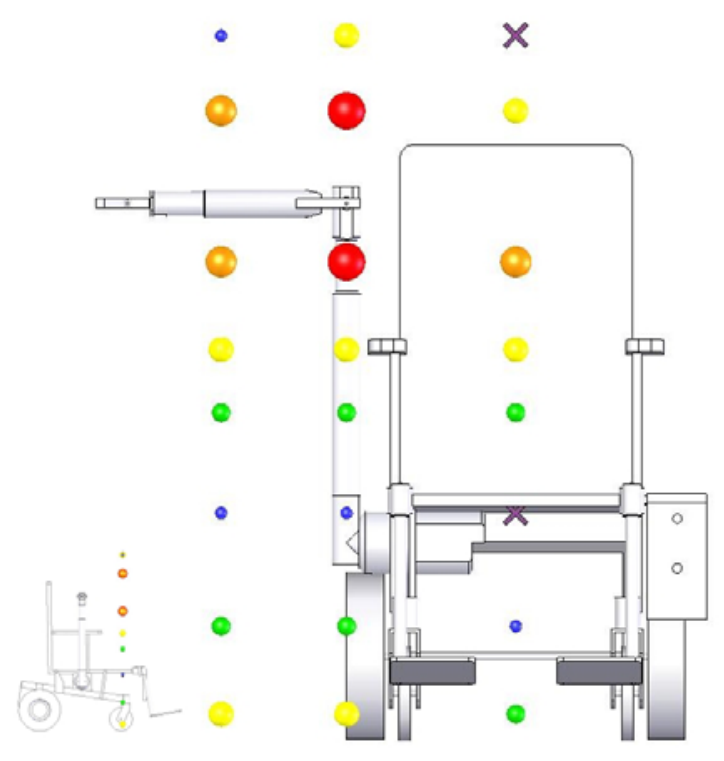

\begin{tabular}{|c|c|c|c|}
\hline $\mathrm{x}$ & $\mathrm{y}$ & $\mathrm{z}$ & $\mathrm{n}$ \\
\hline 6.75 & 0 & -29.8 & 0.36 \\
\hline 6.75 & -13.5 & -29.8 & 0.49 \\
\hline 6.75 & -23.5 & -29.8 & 0.45 \\
\hline 6.75 & 0 & -22.8 & 0.06 \\
\hline 6.75 & -13.5 & -22.8 & 0.26 \\
\hline 6.75 & -23.5 & -22.8 & 0.23 \\
\hline 6.75 & 0 & -13.8 & 0.00 \\
\hline 6.75 & -13.5 & -13.8 & 0.19 \\
\hline 6.75 & -23.5 & -13.8 & 0.16 \\
\hline 6.75 & 0 & -5.82 & 0.23 \\
\hline 6.75 & -13.5 & -5.82 & 0.37 \\
\hline 6.75 & -23.5 & -5.82 & 0.34 \\
\hline 6.75 & 0 & -0.8 & 0.42 \\
\hline 6.75 & -13.5 & -0.8 & 0.56 \\
\hline 6.75 & -23.5 & -0.8 & 0.51 \\
\hline 6.75 & 0 & 6.18 & 0.63 \\
\hline 6.75 & -13.5 & 6.18 & 0.84 \\
\hline 6.75 & -23.5 & 6.18 & 0.75 \\
\hline 6.75 & 0 & 18.2 & 0.44 \\
\hline 6.75 & -13.5 & 18.2 & 0.92 \\
\hline 6.75 & -23.5 & 18.2 & 0.68 \\
\hline 6.75 & 0 & 24.18 & 0.00 \\
\hline 6.75 & -13.5 & 24.18 & 0.46 \\
\hline 6.75 & -23.5 & 24.18 & 0.02 \\
\hline & & & \\
\hline
\end{tabular}

Figure 4.20 : Raptor y-z Plane @ $\mathrm{x}=6.75$ "

In order to gain the highest manipulability measure in the low cabinets, the operator will have to approach the cabinet shelf from the side. This yz-plane is represented in Figure 4.21. The measure at this point is only moderate but this is the highest value available. Access to objects lower than a kitchen counter begins to diminish due to a singularity of the arm at the height of an electric socket $\left(z=-13.8^{\prime \prime}\right)$. 
Table 4.21 : Data for Raptor at $\mathrm{x}=0.54$ ”

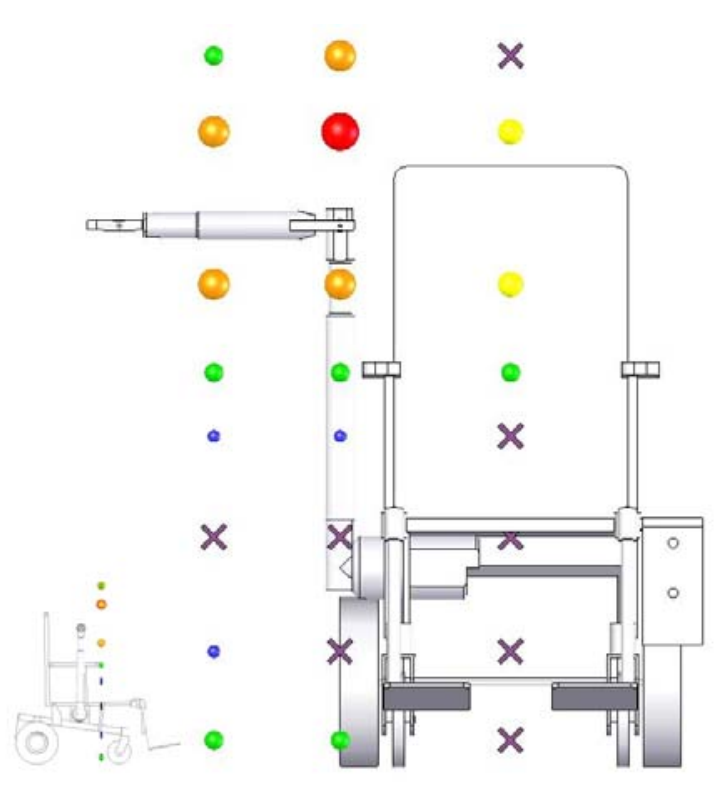

\begin{tabular}{|c|c|c|c|}
\hline $\mathrm{x}$ & $\mathrm{y}$ & $\mathrm{z}$ & $\mathrm{n}$ \\
\hline 0.54 & 0 & -29.8 & 0.00 \\
\hline 0.54 & -13.5 & -29.8 & 0.29 \\
\hline 0.54 & -23.5 & -29.8 & 0.27 \\
\hline 0.54 & 0 & -22.8 & 0.00 \\
\hline 0.54 & -13.5 & -22.8 & 0.00 \\
\hline 0.54 & -23.5 & -22.8 & 0.13 \\
\hline 0.54 & 0 & -13.8 & 0.00 \\
\hline 0.54 & -13.5 & -13.8 & 0.00 \\
\hline 0.54 & -23.5 & -13.8 & 0.00 \\
\hline 0.54 & 0 & -5.82 & 0.00 \\
\hline 0.54 & -13.5 & -5.82 & 0.15 \\
\hline 0.54 & -23.5 & -5.82 & 0.10 \\
\hline 0.54 & 0 & -0.8 & 0.24 \\
\hline 0.54 & -13.5 & -0.8 & 0.38 \\
\hline 0.54 & -23.5 & -0.8 & 0.35 \\
\hline 0.54 & 0 & 6.18 & 0.55 \\
\hline 0.54 & -13.5 & 6.18 & 0.72 \\
\hline 0.54 & -23.5 & 6.18 & 0.65 \\
\hline 0.54 & 0 & 18.2 & 0.55 \\
\hline 0.54 & -13.5 & 18.2 & 0.97 \\
\hline 0.54 & -23.5 & 18.2 & 0.77 \\
\hline 0.54 & 0 & 24.18 & 0.00 \\
\hline 0.54 & -13.5 & 24.18 & 0.63 \\
\hline 0.54 & -23.5 & 24.18 & 0.31 \\
\hline & & & \\
\hline
\end{tabular}

Figure 4.21 : Raptor y-z Plane @ $\mathrm{x}=0.54$ ”

The Raptor has its highest manipulability measure at the point $[0,-13.5,16.5]$ which is shown in Figure 4.22. The ability to manipulate objects is excellent toward the operator's right side but rapidly diminishes once the centerline of the arm is reached. Objects slightly above the lap of the operator have a limited manipulability measure. The manipulator is unable to access an object to the left of the centerline $(y=0)$ of the wheelchair.

Two of the most important activities of daily living are eating and drinking. Because of the significance of these actions, two planes are created specifically to evaluate manipulator effectiveness near the mouth and head (Figure 4.22 and Figure 
4.23). The density of the data points is increased and extends beyond the centerline of the chair. Both figures show that the manipulability measure is very high toward the outer edge of the defined workspace to both heights $(\mathrm{z}=13.5$ " and $\mathrm{z}=16.5$ ") and is still very good at the operator's mouth.

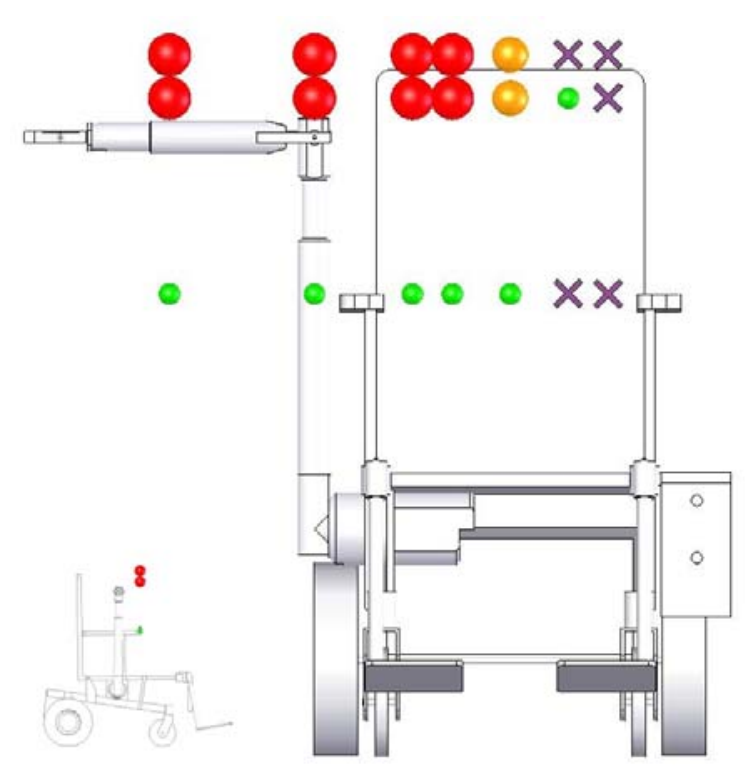

Table 4.22 : Data for Raptor at $\mathrm{x}=0$ "

Figure 4.22 : Raptor y-z Plane @ $\mathrm{x}=0$ ”"

\begin{tabular}{|c|c|c|c|}
\hline $\mathbf{x}$ & $\mathbf{y}$ & $\mathbf{z}$ & $\mathbf{n}$ \\
\hline 0 & 6.75 & 0 & 0.00 \\
\hline 0 & 4 & 0 & 0.00 \\
\hline 0 & 0 & 0 & 0.28 \\
\hline 0 & -4 & 0 & 0.38 \\
\hline 0 & -6.75 & 0 & 0.40 \\
\hline 0 & -13.5 & 0 & 0.40 \\
\hline 0 & -23.5 & 0 & 0.38 \\
\hline 0 & 6.75 & 13.5 & 0.00 \\
\hline 0 & 4 & 13.5 & 0.27 \\
\hline 0 & 0 & 13.5 & 0.68 \\
\hline 0 & -4 & 13.5 & 0.85 \\
\hline 0 & -6.75 & 13.5 & 0.92 \\
\hline 0 & -13.5 & 13.5 & 0.98 \\
\hline 0 & -23.5 & 13.5 & 0.84 \\
\hline 0 & 6.75 & 16.5 & 0.00 \\
\hline 0 & 4 & 16.5 & 0.00 \\
\hline 0 & 0 & 16.5 & 0.63 \\
\hline 0 & -4 & 16.5 & 0.84 \\
\hline 0 & -6.75 & 16.5 & 0.92 \\
\hline 0 & -13.5 & 16.5 & 1.00 \\
\hline 0 & -23.5 & 16.5 & 0.82 \\
\hline
\end{tabular}




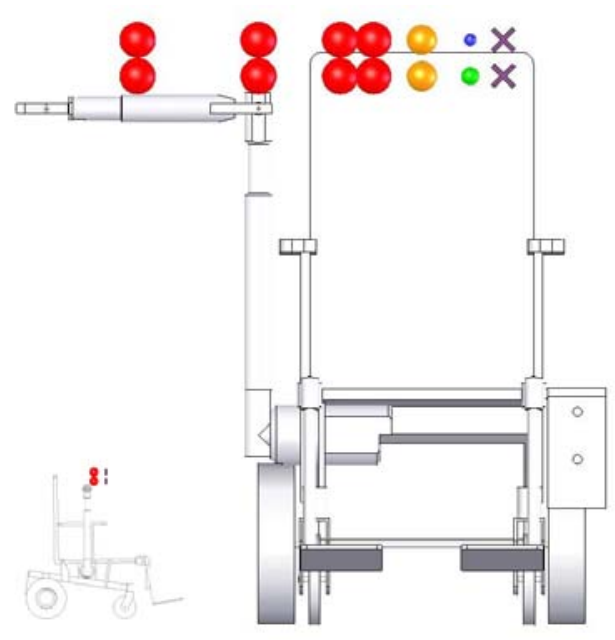

Figure 4.23 : Raptor y-z Plane @ $\mathrm{x}=-4 "$
Table 4.23 : Data for Raptor at $\mathrm{x}=-4$ "

\begin{tabular}{|c|c|c|c|}
\hline $\mathbf{x}$ & $\mathbf{y}$ & $\mathbf{z}$ & $\mathbf{n}$ \\
\hline-4 & 6.75 & 13.5 & 0.00 \\
\hline-4 & 4 & 13.5 & 0.28 \\
\hline-4 & 0 & 13.5 & 0.68 \\
\hline-4 & -4 & 13.5 & 0.84 \\
\hline-4 & -6.75 & 13.5 & 0.91 \\
\hline-4 & -13.5 & 13.5 & 0.97 \\
\hline-4 & -23.5 & 13.5 & 0.83 \\
\hline-4 & 6.75 & 16.5 & 0.00 \\
\hline-4 & 4 & 16.5 & 0.01 \\
\hline-4 & 0 & 16.5 & 0.64 \\
\hline-4 & -4 & 16.5 & 0.85 \\
\hline-4 & -6.75 & 16.5 & 0.93 \\
\hline-4 & -13.5 & 16.5 & 1.00 \\
\hline-4 & -23.5 & 16.5 & 0.83 \\
\hline
\end{tabular}

\subsubsection{Horizontal Planes}

Starting from the plane of a low kitchen shelf, Figure 4.24 shows that the Raptor is able to reach objects from the side of the wheelchair directly to the right of the operator. Although these points can be reached, the access to them is minimal.

The average $n$-value of the horizontal plane is very limited at 0.12 . 


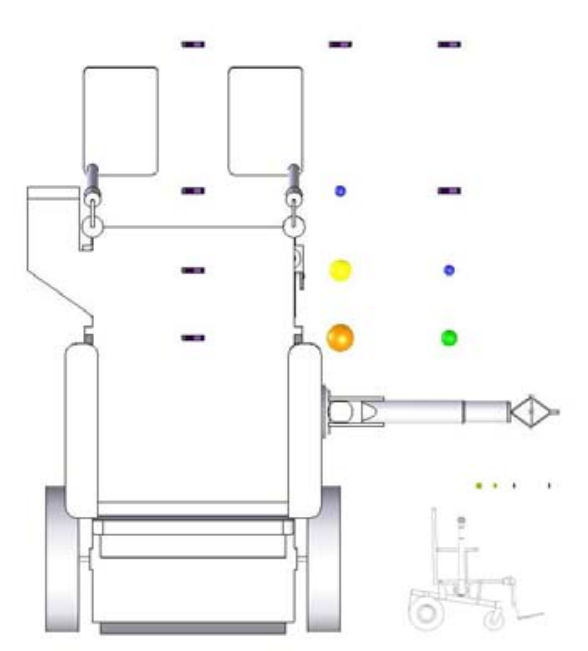

Figure 4.24 : Raptor x-y Plane @ z = 24.18”
Table 4.24 : Data for Raptor $\mathrm{z}=24.18$ ”

\begin{tabular}{|c|c|c|c|}
\hline $\mathbf{x}$ & $\mathbf{y}$ & $\mathbf{z}$ & $\mathbf{n}$ \\
\hline 27.54 & 0 & 24.18 & 0.00 \\
\hline 27.54 & -13.5 & 24.18 & 0.00 \\
\hline 27.54 & -23.5 & 24.18 & 0.00 \\
\hline 14.04 & 0 & 24.18 & 0.00 \\
\hline 14.04 & -13.5 & 24.18 & 0.04 \\
\hline 14.04 & -23.5 & 24.18 & 0.00 \\
\hline 6.75 & 0 & 24.18 & 0.00 \\
\hline 6.75 & -13.5 & 24.18 & 0.46 \\
\hline 6.75 & -23.5 & 24.18 & 0.02 \\
\hline 0.54 & 0 & 24.18 & 0.00 \\
\hline 0.54 & -13.5 & 24.18 & 0.63 \\
\hline 0.54 & -23.5 & 24.18 & 0.31 \\
\hline
\end{tabular}

At the level of a wall-mounted light switch the manipulability measure increases compared to the highest plane. The gripper is able to reach a light switch both from directly in front of the arm and to the side of the chair. Access to objects to the side of the operator is good. This would be the preferred wheelchair orientation when attempting to actuate a light switch.

Figure 4.25 shows that access to the light switch directly in front of the arm is limited, while along the side of the chair the manipulability measures are very good.

The average $n$-value for this plane is good at 0.48 . 


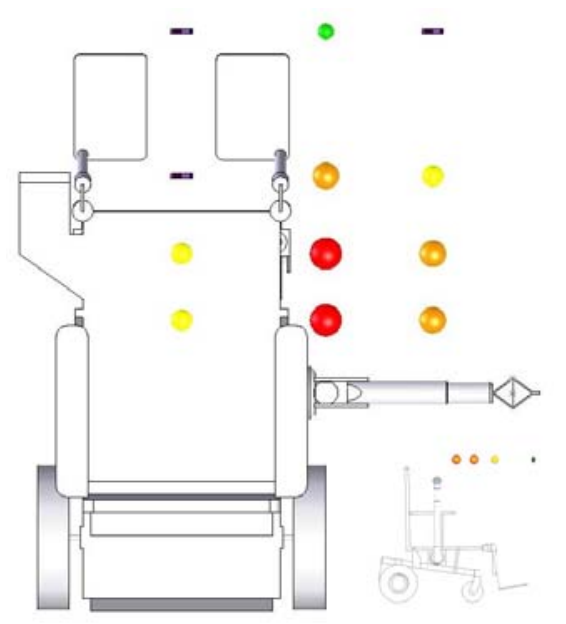

Figure 4.25 : Raptor x-y Plane @ z=18.2”
Table 4.25 : Data for Raptor $\mathrm{z}=18.2$ "

\begin{tabular}{|c|c|c|c|}
\hline $\mathbf{x}$ & $\mathbf{y}$ & $\mathbf{z}$ & $\mathbf{n}$ \\
\hline 27.54 & 0 & 18.2 & 0.00 \\
\hline 27.54 & -13.5 & 18.2 & 0.24 \\
\hline 27.54 & -23.5 & 18.2 & 0.00 \\
\hline 14.04 & 0 & 18.2 & 0.00 \\
\hline 14.04 & -13.5 & 18.2 & 0.72 \\
\hline 14.04 & -23.5 & 18.2 & 0.42 \\
\hline 6.75 & 0 & 18.2 & 0.44 \\
\hline 6.75 & -13.5 & 18.2 & 0.92 \\
\hline 6.75 & -23.5 & 18.2 & 0.68 \\
\hline 0.54 & 0 & 18.2 & 0.55 \\
\hline 0.54 & -13.5 & 18.2 & 0.97 \\
\hline 0.54 & -23.5 & 18.2 & 0.77 \\
\hline
\end{tabular}

At kitchen countertop level (Figure 4.26) the Raptor has very good to excellent access to the sides of the chair, while access to the front of the chair is moderate. At this point, the gripper is unable to reach directly in front of the operator.

Access to objects to the side of the wheelchair is very good to excellent. This is the highest plane that the manipulator can reach an object in the far right corner of the workspace.

Overall the plane has the highest average manipulability measures of all the horizontal planes analyzed at 0.64 with a very good qualitative assessment. This is the maximum average n-value for any horizontal plane. 


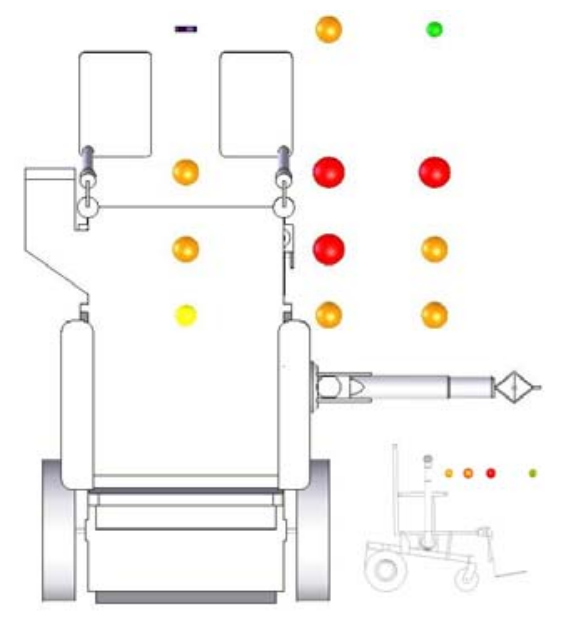

Figure 4.26 : Raptor x-y Plane @ z=6.18”
Table 4.26 : Data for Raptor $\mathrm{z}=6.18$ "

\begin{tabular}{|c|c|c|c|}
\hline $\mathbf{x}$ & $\mathbf{y}$ & $\mathbf{z}$ & $\mathbf{n}$ \\
\hline 27.54 & 0 & 6.18 & 0.00 \\
\hline 27.54 & -13.5 & 6.18 & 0.66 \\
\hline 27.54 & -23.5 & 6.18 & 0.35 \\
\hline 14.04 & 0 & 6.18 & 0.68 \\
\hline 14.04 & -13.5 & 6.18 & 0.98 \\
\hline 14.04 & -23.5 & 6.18 & 0.83 \\
\hline 6.75 & 0 & 6.18 & 0.63 \\
\hline 6.75 & -13.5 & 6.18 & 0.84 \\
\hline 6.75 & -23.5 & 6.18 & 0.75 \\
\hline 0.54 & 0 & 6.18 & 0.55 \\
\hline 0.54 & -13.5 & 6.18 & 0.72 \\
\hline 0.54 & -23.5 & 6.18 & 0.65 \\
\hline
\end{tabular}

The Raptor is able to reach objects directly in front of the operator at the height of a standard table (Figure 4.27). At this elevation and below, the manipulator has limited access to objects directly in front of the operator and the manipulator is able to access all twelve points in the workspace. This is the only plane above the seat of the wheelchair that this occurs.

To open a door the operator would have a greatest chance for success by approaching the door directly in front of the manipulator $(y=-13.5$ "). The average $n$ value for this plane is good at 0.55 .

Manipulability is at its highest level on this plane at $\mathrm{x}=27.54$ and decreases steadily as you approach $\mathrm{x}=0.54$ except for the $\mathrm{x}-\mathrm{z}$ plane at $\mathrm{y}=0$. 


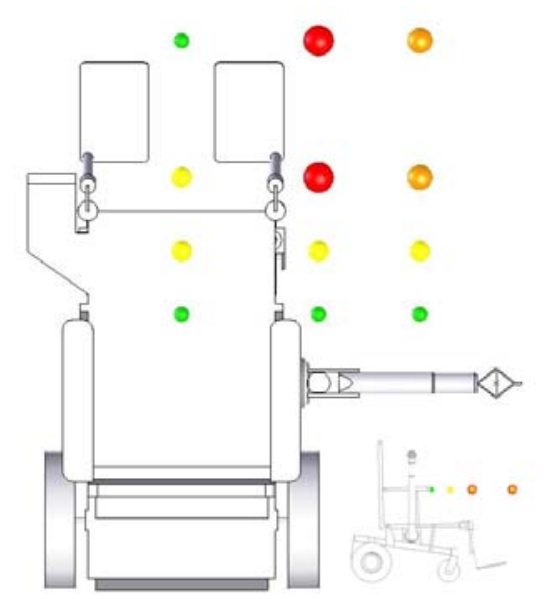

Figure 4.27 : Raptor x-y Plane @ $\mathrm{z}=-0.8$

Table 4.27 : Data for Raptor $\mathrm{z}=-0.8$ "

\begin{tabular}{|c|c|c|c|}
\hline $\mathbf{x}$ & $\mathbf{y}$ & $\mathbf{z}$ & $\mathbf{n}$ \\
\hline 27.54 & 0 & -0.8 & 0.40 \\
\hline 27.54 & -13.5 & -0.8 & 0.90 \\
\hline 27.54 & -23.5 & -0.8 & 0.66 \\
\hline 14.04 & 0 & -0.8 & 0.62 \\
\hline 14.04 & -13.5 & -0.8 & 0.82 \\
\hline 14.04 & -23.5 & -0.8 & 0.73 \\
\hline 6.75 & 0 & -0.8 & 0.42 \\
\hline 6.75 & -13.5 & -0.8 & 0.56 \\
\hline 6.75 & -23.5 & -0.8 & 0.51 \\
\hline 0.54 & 0 & -0.8 & 0.24 \\
\hline 0.54 & -13.5 & -0.8 & 0.38 \\
\hline 0.54 & -23.5 & -0.8 & 0.35 \\
\hline
\end{tabular}

At the height of a coffee table (Figure 4.28) access to all points in front of the operator is good to excellent. The ability to reach objects to the side of the operator continues to decrease. The average n-value of the plane is good at 0.44 . 


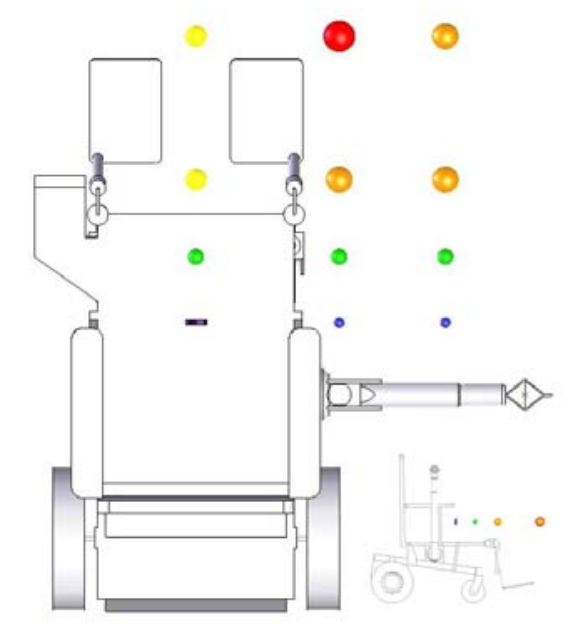

Figure 4.28 : Raptor x-y Plane @ z = - 5.82”
Table 4.28: Data for Raptor $\mathrm{z}=-5.82$ ”

\begin{tabular}{|c|c|c|c|}
\hline $\mathbf{x}$ & $\mathbf{y}$ & $\mathbf{z}$ & $\mathbf{n}$ \\
\hline 27.54 & 0 & -5.82 & 0.53 \\
\hline 27.54 & -13.5 & -5.82 & 0.97 \\
\hline 27.54 & -23.5 & -5.82 & 0.76 \\
\hline 14.04 & 0 & -5.82 & 0.53 \\
\hline 14.04 & -13.5 & -5.82 & 0.70 \\
\hline 14.04 & -23.5 & -5.82 & 0.63 \\
\hline 6.75 & 0 & -5.82 & 0.23 \\
\hline 6.75 & -13.5 & -5.82 & 0.37 \\
\hline 6.75 & -23.5 & -5.82 & 0.34 \\
\hline 0.54 & 0 & -5.82 & 0.00 \\
\hline 0.54 & -13.5 & -5.82 & 0.15 \\
\hline 0.54 & -23.5 & -5.82 & 0.10 \\
\hline
\end{tabular}

Access to objects to the side of the chair is at its lowest point value at $\mathrm{z}=-13.8$ " (Figure 4.29). This is due primarily to the link geometry, the first link is 27 ' long and the second is 17.5 " long, their difference of 9.5 " means that the Raptor is incapable reaching objects that are closer than 9.5 " to the frame $0[-6.26,-13.5,-16.18]$.

The average n-value (0.36) for this plane is limited which is the lowest of all the horizontal planes evaluated. 


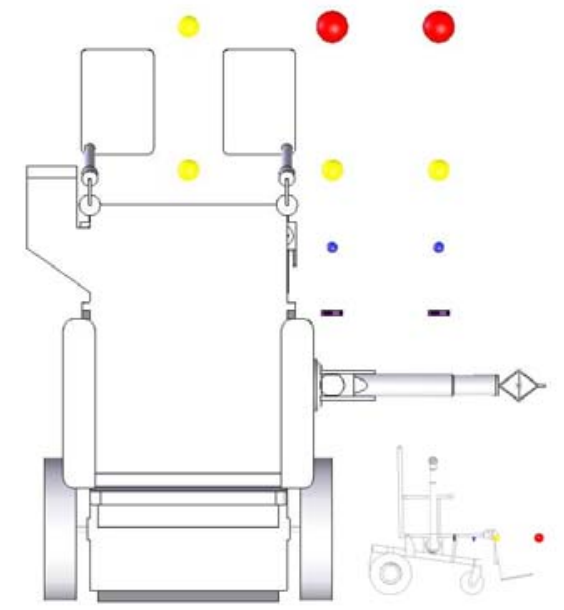

Figure 4.29 : Raptor x-y Plane @ z = - 13.8”
Table 4.29 : Data for Raptor $\mathrm{z}=-13.8 "$

\begin{tabular}{|c|c|c|c|}
\hline $\mathbf{x}$ & $\mathbf{y}$ & $\mathbf{z}$ & $\mathbf{n}$ \\
\hline 27.54 & 0 & -13.8 & 0.60 \\
\hline 27.54 & -13.5 & -13.8 & 0.99 \\
\hline 27.54 & -23.5 & -13.8 & 0.80 \\
\hline 14.04 & 0 & -13.8 & 0.44 \\
\hline 14.04 & -13.5 & -13.8 & 0.57 \\
\hline 14.04 & -23.5 & -13.8 & 0.53 \\
\hline 6.75 & 0 & -13.8 & 0.00 \\
\hline 6.75 & -13.5 & -13.8 & 0.19 \\
\hline 6.75 & -23.5 & -13.8 & 0.16 \\
\hline 0.54 & 0 & -13.8 & 0.00 \\
\hline 0.54 & -13.5 & -13.8 & 0.00 \\
\hline 0.54 & -23.5 & -13.8 & 0.00 \\
\hline
\end{tabular}

Near the ground, the manipulability measure does not change significantly for high and low objects on the floor (Figure 4.30 and Figure 4.31). Low objects on the floor can be accessed from the front as well as the side of the chair.

Along the side of the wheelchair the manipulability measure drops the farther back the object is and objects directly to the right of the operator is very limited.

The average $n$-value for this plane is limited at 0.39 . 


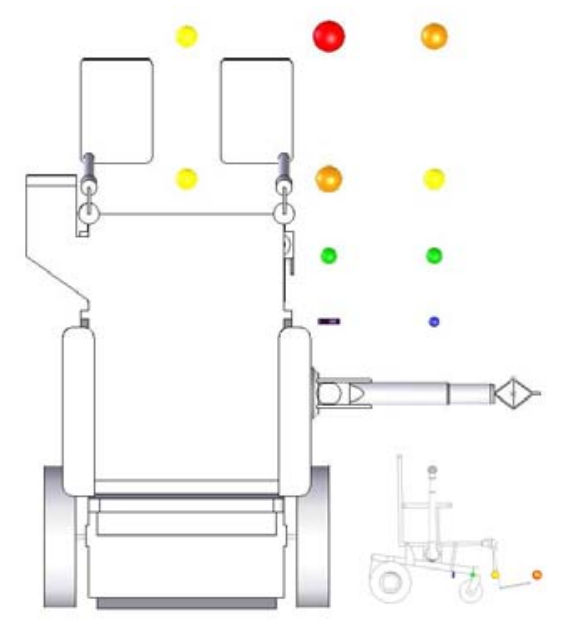

Figure 4.30 : Raptor x-y Plane @ z = - 22.8"
Table 4.30 : Data for $\mathrm{z}=-22.8$ "

\begin{tabular}{|c|c|c|c|}
\hline $\mathbf{x}$ & $\mathbf{y}$ & $\mathbf{z}$ & $\mathbf{n}$ \\
\hline 27.54 & 0 & -22.8 & 0.58 \\
\hline 27.54 & -13.5 & -22.8 & 0.98 \\
\hline 27.54 & -23.5 & -22.8 & 0.79 \\
\hline 14.04 & 0 & -22.8 & 0.48 \\
\hline 14.04 & -13.5 & -22.8 & 0.62 \\
\hline 14.04 & -23.5 & -22.8 & 0.57 \\
\hline 6.75 & 0 & -22.8 & 0.06 \\
\hline 6.75 & -13.5 & -22.8 & 0.26 \\
\hline 6.75 & -23.5 & -22.8 & 0.23 \\
\hline 0.54 & 0 & -22.8 & 0.00 \\
\hline 0.54 & -13.5 & -22.8 & 0.00 \\
\hline 0.54 & -23.5 & -22.8 & 0.13 \\
\hline
\end{tabular}

An interesting note is that there is an apparent horizontal line of symmetry approximately between $\mathrm{z}=-22.8$ " and $\mathrm{z}=-5.82$ '. The true plane of symmetry would be the horizontal plane that the first rotational axis rests $(z=-16.18)$.

The average $n$-value for this plane is good at 0.50 with the highest point within the workspace is at $(27.54,-13.5)$. 


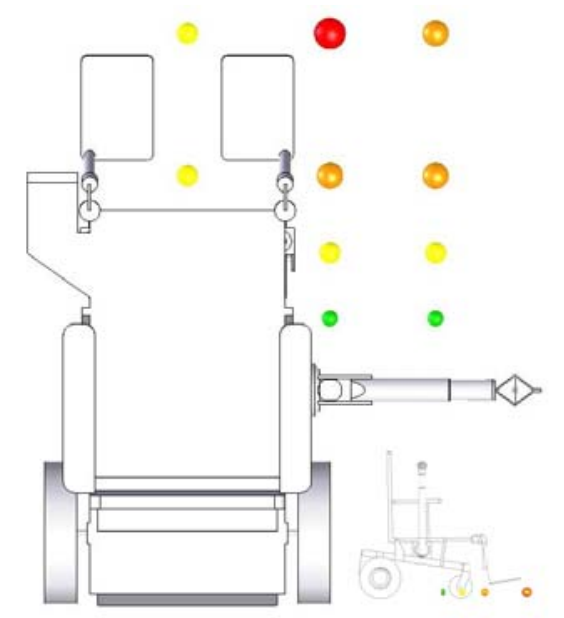

Figure 4.31 : Raptor x-y Plane @ z= -29.8”

Table 4.31 : Data for Raptor at $\mathrm{z}=-29.8$ "

\begin{tabular}{|c|c|c|c|}
\hline $\mathbf{x}$ & $\mathbf{y}$ & $\mathbf{z}$ & $\mathbf{n}$ \\
\hline 27.54 & 0 & -29.8 & 0.46 \\
\hline 27.54 & -13.5 & -29.8 & 0.93 \\
\hline 27.54 & -23.5 & -29.8 & 0.70 \\
\hline 14.04 & 0 & -29.8 & 0.59 \\
\hline 14.04 & -13.5 & -29.8 & 0.78 \\
\hline 14.04 & -23.5 & -29.8 & 0.70 \\
\hline 6.75 & 0 & -29.8 & 0.36 \\
\hline 6.75 & -13.5 & -29.8 & 0.49 \\
\hline 6.75 & -23.5 & -29.8 & 0.45 \\
\hline 0.54 & 0 & -29.8 & 0.00 \\
\hline 0.54 & -13.5 & -29.8 & 0.29 \\
\hline 0.54 & -23.5 & -29.8 & 0.27 \\
\hline
\end{tabular}

A summary of the Raptor's effectiveness in reaching areas common in activities of daily living (ADL) is shown in Table 4.32. This table shows the task, the average manipulability measure of all possible wheelchair orientations that could achieve the task, and the qualitative assessment.

Table 4.32 : Qualitative Summary of Raptor Effectiveness

$\begin{array}{lll}\text { Pick-up off ground } & 0.57 & \text { good } \\ \text { Coffee table } & 0.55 & \text { good } \\ \text { Door knob } & 0.59 & \text { good } \\ \text { Kitchen countertop } & 0.54 & \text { good } \\ \text { Light switch } & 0.35 & \text { limited } \\ \text { Low kitchen shelf } & 0.05 & \text { very limited } \\ \text { Reach into lap } & 0.31 & \text { limited } \\ \text { Access to mouth } & 0.55 & \text { good }\end{array}$


Overall, the average normalized manipulability measure $(n)$ for all the eight tasks in the Table 4.32 is good (0.44). For the tasks that involved reaching onto planes lower than a kitchen countertop oriented from the front or side, the Raptor was close to very good on the qualitative scale $(0.54 \sim 0.59)$.

Access to the lap of the operator was limited (0.31) and the values diminished as the goal moved closer to the operator. Additionally, the inability of the gripper to reach areas to the opposite of the centerline of the wheelchair limited the effectiveness of the arm in the operator's lap.

Reaching a light switch had limited access (0.35). This was due to the low normalized manipulability measures in front of the wheelchair. Access to the side of the operator was very good and as long as this wheelchair orientation was possible, the qualitative accessibility would be very good (0.62).

The area least able to be reached is a low kitchen shelf. The Raptor has very limited access and was only able to reach the shelf in two of the six possible outer perimeter data points. 


\section{Chapter Five Design Insights and Recommendations}

\section{$5.1 \quad$ Design Insights}

Both of the commercially-available systems analyzed were able to pick up objects from the floor, reach into the operator's immediate workspace, and manipulate objects on cabinets, desks and low tables. In several cases the manipulators are only able to manipulate objects in certain planes from specific orientation of the chair. For example, when attempting to retrieve objects off the floor, the Raptor is near its maximum manipulability measure from the front while it is incapable of retrieving an object directly to the right of the user. This would require the operator to position the chair in a specific orientation before attempting to manipulate an object with the arm.

Additionally, the commercial designs appear to be well thought out, designed and manufactured. Through the analysis of the manipulability measure in a rehabilitationspecific workspace, it has been shown that there are areas of weakness in both designs that can be improved. Several design insights and recommendations are noted below.

- The Manus has very low manipulability measures at or very near the ground and must orient the chair with respect to the object in order to manipulate it.

- The Manus would be more effective in retrieving objects from the floor if the mounting base were installed lower on the wheelchair with respect to the floor. The additional height increase provided by the z-axis lift device would compensate for its lower initial position.

- The front mounting of the Manus provides excellent access to objects in front and above the operator. The vertical reach of the manipulator is very good even without using the z-axis lift device. All analysis was done with the Z-axis lift in its lowest position. 
- Given the size of the workspace, the closer the object is to the first axis of rotation of the manipulator, the lower the manipulability measure will be.

- Increasing the number of DOF of the manipulator will allow better access to some internal regions (near singularities) but this will come at the expense of one or more DOF's of gripper orientation.

- Low mounting position and link geometry provides the Raptor with very good access to objects low to the ground and items directly in front of the wheelchair.

- The Raptor has very low manipulability when reaching into kitchen cabinets. The side-mount design limits how high that Raptor can be mounted with respect to the floor. Increasing the first link length could allow higher reach.

- A plane of symmetry exists at a horizontal plane where the first rotational axis rests. This plane of symmetry relates manipulability measures above and below $\mathrm{z}$ $=16.26 "$ (user frame).

- Accessibility on horizontal planes is still good up to $\mathrm{z}=6.18$. This is almost twice the distance that the manipulator's primary axis of rotation is above the floor.

- The length of the final link limits the Raptor's ability to cross the centerline of the wheelchair. Objects on the lap of the operator become increasingly difficult to manipulate as one approaches the centerline of the wheelchair.

- The joint angle range in the third joint reduces the manipulator effectiveness. The arm cannot effectively reach back onto itself. The arm is not capable of reaching objects near the first joint motor.

- The closer the goal is to the manipulator's primary axis of rotation, the lower the manipulability measure. This is magnified by the limited joint angle range in the third revolute joint.

- The highest manipulability measure for the Raptor for all horizontal planes up to $\mathrm{z}$ $=-0.8$ " is at $(27.54,-13.5)$. 


\subsection{Design Recommendations}

There is no single mounting location that will allow equal potential of manipulability for all activities of daily living. Front mounting of an arm interferes with the user's ability to maneuver under desks and sinks and is a visual obstruction to social interaction. Side mounting increases the width of the wheelchair, making movement through doors and hallways more difficult. Mounting the arm in the rear of the chair requires longer manipulator link lengths to reach in front of the chair and this increases the loads on the motors and bearings. A single fixed mounting location does not allow access throughout the entire workspace.

From the evaluation of the two commercial WMRAs, improvements to these commercially-available arms could come in two ways. First would be rail-mounting, allowing for front- and side-locking positions, along with a rear-locking position for the manipulator base. In this way, the manipulator could benefit from using the optimum mounting location for completing a specific ADL.

There are a variety of uses for a manipulator in assistive rehabilitation applications. Additionally, a well-designed robotic arm could be mounted onto a variety of surfaces and locations if the arm did not have fixed link geometry. In this way, a WMRA could be used in a workstation application with only minor changes to link lengths and controller settings. Commercially-available WMRA are designed primarily for mounting onto wheelchairs and are not easily used as workstation manipulators. These arms cannot be made to fit a workstation by the end user. This limits the use of the manipulator and reduces its potential use in a home or office environment.

The second enhancement would be to design the manipulator as a system of modular joints and link lengths. A modular manipulator design would allow the system to be adjusted for various geometries. This would allow the system to be applied to stationary applications as well as mobile mounts. The modularity of the manipulator will apply both to the links and joints as well as the rail track mount. 


\subsubsection{Rail Mounted Manipulator}

The first and most novel improvement for a WMRA is a side-mounted railpositioned manipulator base that is capable of multiple base positions while in use. This design uses the concept of having the manipulator move along a track. This was first developed with DeVAR, an early workstation robot design. At the time of this research, this mounting track method has not been applied to WMRAs.

The base of the manipulator would rest on a detachable track that runs along the side of the chair from the front to the back. The entire shape of the rail would be roughly $\mathrm{C}$-shaped with curved bends at either end. The base could then move along this track to three discrete points from which to operate. Additionally, the rail would need to have provisions for quick release, facilitating ease of transfer to and from the wheelchair.

There would be three positions for the base of the manipulator. The first would be in front of the chair and would allow access to objects in front and center of the operator, the second position would be a side mount that is slightly behind the yz plane of the operator which would allow for side access and for feeding, the final position is a stow position which would allow the arm to be folded away when not in use. The front lock location would be analogous to the Manus mount while the second lock location would be near the Raptor position.

Link geometries would be critical for the success of the design. There would need to be a compromise in the link geometry between the requirements for each base position. Each base position would have specific link lengths that would provide optimum workspace for the operator and the final link geometries of the system would be a compromise between the two sub-systems requirements.

It may also be beneficial for the track to have a 90-degree twist in the track. In the side-mount location, the arm has its first rotational axis in the y direction and the frontmount location has a first rotational axis in the $\mathrm{z}$ direction. This change in the first rotational axis may pose too significant a restriction on the link length geometries. The

optimum link geometries for each base position may actually compete against each other, making the final compromise manipulator unacceptably inefficient. 
The design provision of a track-mounted manipulator arm would allow for the user to move under desks and still use the manipulator arm in the side mount mode or move through doors and confined areas with the manipulator arm, stowed in the rear position, folded up safely behind the wheelchair.

\subsubsection{Reconfigurable Manipulator}

The other design recommendation for the manipulator is that it be reconfigurable, modular, and aesthetically pleasing to the user. Reconfigurability would be a beneficial aspect for the robotic arm because the real world cannot be rearranged so that a fixed manipulator can be more efficient. Often it is not possible to mount a manipulator in the optimum location for its intended task. This was the case with the mounting of the Raptor arm. The power wheelchair's structure did not allow the motor to be affixed as high as necessary for an optimum mounting location.

Individual arm link lengths can be modified to create a robotic arm workspace that matches actual workspace requirements. Because both power wheelchairs and workspaces vary in size and construction, a modular approach to link lengths design and joint construction should be utilized. This modularity would allow the installer to custom tailor the links to better fit the mounting location.

Modularity is addressed through the use of easily-altered revolute joints which allow for changes to the number of degrees of freedom in the arm with only simple change of components. Thus the arm can be designed around the user's requirements and not vice versa. Controller design would incorporate configuration changes and compute inverse kinematics of the revised design.

Finally, a design that can be used in several applications would provide a greater function per dollar by allowing one general manipulator to serve multiple roles. A reconfigurable modular manipulator could be mounted on a wheelchair, mobile base, or within a workstation. The increased functionality of the arm could allow for improved market share. The potential benefit from the higher production rates would be a decrease in cost of the manipulator. 


\section{Chapter Six Summary and Future Work}

There has been significant progress in bringing commercially-viable WMRAs into the marketplace in the past 30 years. Of these devices, the Manus arm has seen the most development, though mostly in Europe. There is still much progress to be made in order to minimize user effort and reduce operator fatigue. Over the operational life of WMRAs they are cost-effective yet their high initial cost has been a prohibitive barrier for many users.

\subsection{Design Recommendations and Insights}

Link lengths need to be kept as short as possible to reduce dynamic loads. While longer links allow for greater values of manipulability measure, they require greater encoder precision to achieve the same level of precision as a shorter link.

Mounting the arm closer to the ground biases the arm's ability to manipulating objects close to the ground, desks and countertops. While a rear mount would solve the problems of increased chair width and visual obstruction, it can cause further difficulties with link geometries.

The Raptor design, while very good at manipulating objects in front of the wheelchair, was challenged to reach into the lower cabinet at almost any orientation. The Raptor link geometries could be slightly modified to allow access to low cabinets and provide better access across the centerline of the wheelchair.

The mounting location of the Manus allowed for excellent access to low shelves and desks but suffered from internal singularities which limited its ability to manipulate objects close to its base. The internal singularities caused difficulties for the inverse kinematic program to determine the proper joint angles. Often the start point of the arm had to be changed several times in order for the gripper frame to reach the goal. 


\subsection{Future Work}

There are both software- and hardware-based methods to further the work done in this thesis. The software development would further the inverse kinematic program (robot.m) and the hardware advancement would create virtual manipulators using track mount and modular links. These hardware models would use both the Manus and Raptor as a baseline for comparison.

Modify robot.m to create automatic mesh generation within the workspace. This would determine manipulability measures throughout the workspace with user-defined grid density. The software program that determined the inverse kinematics of the Manus and Raptor had several limitations. The robot.m program required manual entry of the desired gripper position and could only determine one solution at a time. A better study of the entire workspace would be achieved with a denser map of the manipulability measures. For each arm, the smallest grid analyzed was 6.21 " by 10 " by 5.02 " with a total number of 131 data points.

Integrate the inverse kinematic program with Solid WORKS ${ }^{\circledR}$ by creating an interface so that solutions from the inverse kinematic program can be input into Solid WORKS $^{\circledR}$ to create real-time simulations of the arm within the workspace.

Hardware development would be based on the concept of the rail-mounted multiposition manipulator. The use of both operating positions would be fundamental in the analysis because the manipulator should function equally in each position and the sum of the manipulability of both positions should be greater than the manipulability of the commercial designs.

Although only given a cursory look in this thesis, the use of modular links and joints in a reconfigurable design would make a WMRA more versatile so that it could be used in a variety of applications. This flexibility would allow a production arm to fill more niches and increase the number of unit sales, further decreasing unit cost and increasing availability of the unit.

While this thesis has focused solely on a procedure for kinematic analysis and evaluation of manipulators, a manipulator is only a series of motors, encoders, gearboxes, 
wires, and links without a controller. Controller design is a vital consideration when designing a complete robotic manipulator system. The greatest gains for robotic manipulators in rehabilitation applications will come from advanced controllers that are easily programmed and operated. 


\section{References}

${ }^{1}$ US Census Bureau (1993). “Americans with Disabilities”. Statistical Brief SB/94-1, January.

2. R. Cooper: "Rehabilitation Engineering Applied to Mobility and Manipulation", Institute of Physics Publishing, ISBN: 0750303433.

3.. J.B.Reswick, "The Moon over Dubrovnik -- A Tale of Worldwide Impact on Persons with Disabilities," Advances in External Control of Human Extremities, 1990.

4.. J.R. Allen, A. Karchak, Jr., E.L. Bontrager, "Design and Fabrication of a Pair of Rancho Anthropomorphic Arms", Technical Report, The Attending Staff Association of the Rancho Los Amigos Hospital, Inc, 1972.

5. T. Rahman, S. Stroud, R. Ramanathan, M. Alexander, R. Alexander, R. Seliktar, W. Harwin: "Consumer Criteria for an Arm Orthosis", Applied Science and Engineering Laboratories, www95.homepage.villanova.edu/rungun.ramanathan/publications/t_and_d.pdf.

6. H.F. Machiel Van der Loos, "Lessons Learned in the Application of Robotics Technology to the Field of Rehabilitation”, Proc. IEEE '95.

7. M.J. Topping. "The Development of Handy-1, A Robotic System to Assist the Severely Disabled, Proc ICORR '99, 244-249.

${ }^{8}$ M. Topping, H. Heck, G. Bolmsjo, D. Weightman: "The Development of RAIL (Robotic Aid to Independent Living)" Proceedings of the third TIDE Congress. (1998). 
9. M. Hillman, A. Gammie. "The Bath Institute of Medical Engineering Assistive Robot". Proc. ICORR '94, 211-212.

10. J.L. Dallaway, R.D. Jackson: “RAID - A Vocational Robotic Workstation”. Proc. ICORR' 92.

11. Robotic Assistive Device, Neil Squire Foundation, http://www.neilsquire.ca/rd/projects/RobotApp.htm.

12. N. Katevas (Ed): "Mobile Robotics in Health Care Services" IOS Press Amsterdam, 2000, pp. 227-251.

13. H.F.M. Van der Loos, VA/Stanford Rehabilitation Robotics Research and Development Program: "Lessons Learned in the Application of Robotics Technology to the Field of Rehabilitation". IEEE Trans. Rehabilitation Engineering, Vol. 3, No. 1, March, 1995, pp. 46-55.

14. B. Borgerding, O. Ivlev, C. Martens, N. Ruchel: 'FRIEND

Functional Robot Arm with User Friendly Interface for Disabled People“. Institute of Automation Technology (IAT)

http://www.iat.unibremen.de/Projekte/HTML e/FRIEND.htm.

15. S. Sheredos, B. Taylor, C. Cobb, E. Dann: "The Helping Hand Electro-Mechanical Arm”. Proc. RESNA '95, 493-495.

16. G. Bolmsjö, M. Olsson, P. Hedenborn, U. Lorentzon, F. Charnier, H. Nasri: "Modular Robotics Design - System Integration of a Robot for Disables People".

17. H.A. Yanco: "Integrating Robotic Research: A Survey of Robotic Wheelchair Development" AAAI Spring Symposium on Integrating Robotic Research, Stanford, California, March, 1998. 
18. P.R. Warner, S.D. Prior: "Investigations into the Design of a Wheelchair-Mounted Rehabilitation Robotic Manipulator", Proceedings of the 3rd Cambridge Workshop on Rehabilitation Robotics, Cambridge University, England, April 81994.

${ }^{19 .}$ H. Eftring, K. Boschian: “Technical Results from Manus User Trials”, Proc. ICORR '99, 136-141.

${ }^{20 .}$ S.D. Prior, P.R. Warner, J.T. Parsons, and P. Oettinger: "Design and Development of an Electric Wheelchair Mounted Robotic Arm for Use by People with Physical Disabilities.", Transactions of the IMACS/SICE International Symposium on Robotics, Mechatronics \& Manufacturing Systems, Elsevier Science Publishers B.V., ISBN: 0-44489700-3, pp.221-226, July 1993.

${ }^{21 .}$ M. Hillman, K. Hagan, S. Hagan, J. Jepson, R. Orppwood: "A Wheelchair Mounted Assistive Robot”, Proc. ICORR '99, 86-91.

22. S. Prior: "An Electric Wheelchair Mounted Robotic Arm - A Survey of Potential Users". Journal of Medical Engineering \& Technology, B14B:143-154 (1990).

23. J. Craig: "Introduction to Robotics Mechanics and Control", Second Edition, AddisonWesley Publishing, ISBN 0-201-09528-9. 
Appendices 
Appendix A: Manipulability Data

Manus Data from the Inverse kinematics program:

$\mathrm{x}, \mathrm{y}, \mathrm{z}$ are the position vector, inputs

$\mathrm{n}$ is the normalized manipulability measure.

Manip is the actual manipulability measure; no values for the theta columns indicates that the point was unobtainable

\begin{tabular}{|c|c|c|c|c|c|c|c|}
\hline & $\begin{array}{l}\text { desir } \\
\text { posit }\end{array}$ & & & & $\begin{array}{l}\text { required } \\
\text { angles } \\
\text { (radians) }\end{array}$ & & \\
\hline$x$ & $\mathbf{y}$ & $\mathbf{z}$ & $\mathbf{n}$ & Theta 1 & Theta 2 & Theta 3 & Manip \\
\hline 14.04 & 0 & -29.8 & 0.23 & -2.2227 & 2.5172 & 0.6609 & 1546.7 \\
\hline 14.04 & 13.5 & -29.8 & 0.00 & & & & 0 \\
\hline 14.04 & 23.5 & -29.8 & 0.20 & 1.2464 & 2.5616 & 0.3721 & 1344.2 \\
\hline 14.04 & 0 & -22.8 & 0.37 & -2.2235 & 2.0072 & 1.4476 & 2491.4 \\
\hline 14.04 & 13.5 & -22.8 & 0.00 & & & & 0 \\
\hline 14.04 & 23.5 & -22.8 & 0.53 & 1.2464 & 1.9539 & 1.2999 & 3562.2 \\
\hline 14.04 & 0 & -13.8 & 0.33 & -2.2243 & 1.4552 & 2.0769 & 2197.9 \\
\hline 14.04 & 13.5 & -13.8 & 0.00 & & & & 0 \\
\hline 14.04 & 23.5 & -13.8 & 0.51 & 1.2464 & 1.3794 & 1.9203 & 3473.5 \\
\hline 14.04 & 0 & -5.82 & 0.23 & -2.2234 & 0.7967 & 2.491 & 1526.9 \\
\hline 14.04 & 13.5 & -5.82 & 0.00 & & & & 0 \\
\hline 14.04 & 23.5 & -5.82 & 0.41 & 1.2464 & 0.7835 & 2.284 & 2796 \\
\hline 14.04 & 0 & -0.8 & 0.18 & -2.2234 & 0.19999 & 2.6498 & 1190.5 \\
\hline 14.04 & 13.5 & -0.8 & 0.00 & & & & 0 \\
\hline 14.04 & 23.5 & -0.8 & 0.37 & 1.2464 & 0.3467 & 2.4039 & 2486.6 \\
\hline 14.04 & 0 & 6.18 & 0.19 & -2.2234 & -0.519 & 2.6043 & 1290.4 \\
\hline 14.04 & 13.5 & 6.18 & 0.00 & & & & 0 \\
\hline 14.04 & 23.5 & 6.18 & 0.38 & 1.2464 & -0.1684 & 2.3711 & 2574.9 \\
\hline 14.04 & 0 & 18.2 & 0.34 & -2.2234 & -0.6703 & 2.0199 & 2271.2 \\
\hline 14.04 & 13.5 & 18.2 & 0.00 & & & & 0 \\
\hline 14.04 & 23.5 & 18.2 & 0.52 & 1.2464 & -0.4019 & 1.8678 & 3535.1 \\
\hline 14.04 & 0 & 24.18 & 0.37 & -2.2234 & -0.5335 & 1.6115 & 2519.1 \\
\hline 14.04 & 13.5 & 24.18 & 0.00 & & & & 0 \\
\hline 14.04 & 23.5 & 24.18 & 0.54 & 1.2464 & -0.3054 & 1.4699 & 3678.2 \\
\hline
\end{tabular}


Appendix A (Continued)

Manus data from the inverse kinematics program:

\begin{tabular}{|c|c|c|c|c|c|c|c|}
\hline & $\begin{array}{l}\text { desir } \\
\text { posit }\end{array}$ & & & & $\begin{array}{l}\text { required } \\
\text { angles } \\
\text { (radians) }\end{array}$ & & \\
\hline$x$ & $y$ & $\mathbf{z}$ & $\mathbf{n}$ & Theta 1 & Theta 2 & Theta 3 & Manip \\
\hline 6.75 & 0 & -29.8 & 0.25 & 3.6017 & 3.0655 & 5.7658 & 1709.9 \\
\hline 6.75 & 13.5 & -29.8 & 0.21 & 2.1157 & 3.3018 & 5.572 & 1411.7 \\
\hline 6.75 & 23.5 & -29.8 & 0.00 & & & & 0 \\
\hline 6.75 & 0 & -22.8 & 0.50 & -2.6822 & 1.9595 & 1.3277 & 3400.6 \\
\hline 6.75 & 13.5 & -22.8 & 0.32 & 2.1145 & 2.034 & 1.4774 & 2150.4 \\
\hline 6.75 & 23.5 & -22.8 & 0.61 & 1.7827 & 1.9459 & 1.1789 & 4090.2 \\
\hline 6.75 & 0 & -13.8 & 0.47 & 3.5911 & 1.3889 & 1.9597 & 3181.9 \\
\hline 6.75 & 13.5 & -13.8 & 0.27 & 2.1149 & 1.4914 & 2.1138 & 1849.6 \\
\hline 6.75 & 23.5 & -13.8 & 0.64 & 1.7827 & 1.3645 & 1.8008 & 4309.2 \\
\hline 6.75 & 0 & -5.82 & 0.37 & 3.5906 & 0.7761 & 2.3309 & 2503.3 \\
\hline 6.75 & 13.5 & -5.82 & 0.18 & 2.1157 & 0.8212 & 5.5468 & 1211.9 \\
\hline 6.75 & 23.5 & -5.82 & 0.55 & 1.7827 & 0.8093 & 2.1409 & 3725.7 \\
\hline 6.75 & 0 & -0.8 & 0.33 & 3.6007 & 3.2401 & 3.8379 & 2246.7 \\
\hline 6.75 & 13.5 & -0.8 & 0.13 & 2.1157 & 3.6571 & 3.5595 & 878.2 \\
\hline 6.75 & 23.5 & -0.8 & 0.51 & 1.7826 & 3.0423 & 4.0366 & 3453.1 \\
\hline 6.75 & 0 & 6.18 & 0.35 & 3.6009 & 2.6423 & 3.8725 & 2338.5 \\
\hline 6.75 & 13.5 & 6.18 & 0.14 & 2.1157 & 2.7119 & 3.6114 & 978.8 \\
\hline 6.75 & 23.5 & 6.18 & 0.51 & 1.7819 & 2.5687 & 4.0553 & 3466.6 \\
\hline 6.75 & 0 & 18.2 & 0.49 & 3.6069 & 1.694 & 4.3832 & 3298.9 \\
\hline 6.75 & 13.5 & 18.2 & 0.28 & 2.1157 & 1.5904 & 4.2262 & 1908.9 \\
\hline 6.75 & 23.5 & 18.2 & 0.64 & 1.7823 & 1.7173 & 4.5318 & 4345.5 \\
\hline 6.75 & 0 & 24.18 & 0.52 & 3.6067 & 1.3161 & 4.7835 & 3483.6 \\
\hline 6.75 & 13.5 & 24.18 & 0.32 & 2.1157 & 1.2315 & 4.6385 & 2156.9 \\
\hline 6.75 & 23.5 & 24.18 & 0.64 & 1.7823 & 1.3355 & 4.9287 & 4319.4 \\
\hline
\end{tabular}


Appendix A (Continued)

Manus data from the inverse kinematics program:

\begin{tabular}{|c|c|c|c|c|c|c|c|}
\hline & $\begin{array}{l}\text { desir } \\
\text { positi }\end{array}$ & & & & $\begin{array}{l}\text { required } \\
\text { angles } \\
\text { (radians) }\end{array}$ & & \\
\hline$x$ & $y$ & z & $n$ & Theta 1 & Theta 2 & Theta 3 & Manip \\
\hline 0.54 & 0 & -29.8 & 0.00 & & & & 0 \\
\hline 0.54 & 13.5 & -29.8 & 0.12 & 2.5426 & 2.8315 & 6.0801 & 829.4 \\
\hline 0.54 & 23.5 & -29.8 & 0.00 & & & & 0 \\
\hline 0.54 & 0 & -22.8 & 0.65 & 3.4417 & 3.1403 & 5.1939 & 4366.9 \\
\hline 0.54 & 13.5 & -22.8 & 0.57 & 2.5428 & 3.3105 & 5.0395 & 3880.3 \\
\hline 0.54 & 23.5 & -22.8 & 0.66 & 2.1224 & 2.9872 & 5.3586 & 4476.6 \\
\hline 0.54 & 0 & -13.8 & 0.72 & 3.4417 & 3.2729 & 4.5771 & 4894.2 \\
\hline 0.54 & 13.5 & -13.8 & 0.58 & 2.5427 & 3.4566 & 4.4276 & 3938.3 \\
\hline 0.54 & 23.5 & -13.8 & 0.83 & 2.1224 & 3.1246 & 4.7186 & 5627.5 \\
\hline 0.54 & 0 & -5.82 & 0.66 & 3.4416 & 3.1534 & 4.2512 & 4426.6 \\
\hline 0.54 & 13.5 & -5.82 & 0.49 & 2.5427 & 3.3437 & 4.079 & 3309.9 \\
\hline 0.54 & 23.5 & -5.82 & 0.79 & 2.1224 & 3.016 & 4.398 & 5328.9 \\
\hline 0.54 & 0 & -0.8 & 0.62 & 3.4426 & 2.9492 & 4.1506 & 4176 \\
\hline 0.54 & 13.5 & -0.8 & 0.45 & 2.5428 & 3.1103 & 3.9655 & 3006.8 \\
\hline 0.54 & 23.5 & -0.8 & 0.77 & 2.1224 & 2.8226 & 4.3109 & 5182.6 \\
\hline 0.54 & 0 & 6.18 & 0.63 & 3.4424 & 2.5187 & 4.1778 & 4255.1 \\
\hline 0.54 & 13.5 & 6.18 & 0.46 & 2.5428 & 2.5929 & 3.998 & 3104.2 \\
\hline 0.54 & 23.5 & 6.18 & 0.78 & 2.1224 & 2.4436 & 4.3368 & 5246.4 \\
\hline 0.54 & 0 & 18.2 & 0.73 & 3.4423 & 1.7166 & 4.6297 & 4939.4 \\
\hline 0.54 & 13.5 & 18.2 & 0.59 & 2.5427 & 1.7133 & 4.4822 & 4014.5 \\
\hline 0.54 & 23.5 & 18.2 & 0.83 & 2.1224 & 1.6973 & 4.7727 & 5638.9 \\
\hline 0.54 & 0 & 24.18 & 0.70 & 3.4423 & 1.3326 & 5.029 & 4712.8 \\
\hline 0.54 & 13.5 & 24.18 & 0.60 & 2.5427 & 1.3325 & 4.8788 & 4069.4 \\
\hline 0.54 & 23.5 & 24.18 & 0.75 & 2.1224 & 1.3089 & 5.1839 & 5035.1 \\
\hline
\end{tabular}


Appendix A (Continued)

Manus data from the inverse kinematics program:

\begin{tabular}{|c|c|c|c|c|c|c|c|}
\hline & $\begin{array}{l}\text { desire } \\
\text { positio }\end{array}$ & & & & $\begin{array}{l}\text { required } \\
\text { angles } \\
\text { (radians) }\end{array}$ & & \\
\hline $\mathbf{x}$ & $y$ & $\mathbf{z}$ & $\mathbf{n}$ & Theta 1 & Theta 2 & Theta 3 & Manip \\
\hline 0 & -6.75 & 0 & 0.94 & 3.7437 & 2.6441 & 4.5093 & 6330.9 \\
\hline 0 & -4 & 0 & 0.82 & 3.631 & 2.7441 & 4.3607 & 5506.6 \\
\hline 0 & 0 & 0 & 0.65 & 3.4311 & 2.8739 & 4.1812 & 4399.2 \\
\hline 0 & 4 & 0 & 0.53 & 3.1884 & 2.9772 & 4.051 & 3569.8 \\
\hline 0 & 6.75 & 0 & 0.48 & 3.0035 & 3.0244 & 3.9956 & 3221.3 \\
\hline 0 & 13.5 & 0 & 0.48 & 2.5636 & 3.0203 & 4.0003 & 3250.6 \\
\hline 0 & 23.5 & 0 & 0.80 & 2.146 & 2.7594 & 4.3389 & 5376.7 \\
\hline 0 & -6.75 & 13.5 & 0.96 & 3.7438 & 1.9343 & 4.7381 & 6455.5 \\
\hline 0 & -4 & 13.5 & 0.86 & 3.631 & 1.9773 & 4.5951 & 5819 \\
\hline 0 & 0 & 13.5 & 0.73 & 3.4312 & 2.0146 & 4.4311 & 4902 \\
\hline 0 & 4 & 13.5 & 0.62 & 3.1888 & 2.0297 & 4.3143 & 4159 \\
\hline 0 & 6.75 & 13.5 & 0.57 & 3.0035 & 2.0318 & 4.2699 & 3861.3 \\
\hline 0 & 13.5 & 13.5 & 0.58 & 2.5636 & 2.0317 & 4.2738 & 3887.7 \\
\hline 0 & 23.5 & 13.5 & 0.85 & 2.146 & 1.9826 & 4.5758 & 5721.1 \\
\hline 0 & -6.75 & 16.5 & 0.94 & 3.7343 & 1.7599 & 4.8583 & 6329.6 \\
\hline 0 & -4 & 16.5 & 0.87 & 3.631 & 1.793 & 4.7292 & 5854.8 \\
\hline 0 & 0 & 16.5 & 0.75 & 3.4314 & 1.8213 & 4.566 & 5036.8 \\
\hline 0 & 4 & 16.5 & 0.65 & 3.1888 & 1.8281 & 4.456 & 4360.5 \\
\hline 0 & 6.75 & 16.5 & 0.60 & 3.0035 & 1.8267 & 4.4111 & 4061 \\
\hline 0 & 13.5 & 16.5 & 0.61 & 2.5636 & 1.8274 & 4.418 & 4109.5 \\
\hline 0 & 23.5 & 16.5 & 0.85 & 2.146 & 1.7973 & 4.7108 & 5774.9 \\
\hline
\end{tabular}


Appendix A (Continued)

Manus data from the inverse kinematics program:

\begin{tabular}{lll}
\multicolumn{3}{c}{ desired } \\
$\mathbf{3}$ & position \\
$\mathbf{x}$ & $\mathbf{y}$ & $\mathbf{z}$ \\
-4 & -6.75 & 13.5 \\
-4 & -4 & 13.5 \\
-4 & 0 & 13.5 \\
-4 & 4 & 13.5 \\
-4 & 6.75 & 13.5 \\
-4 & 13.5 & 13.5 \\
-4 & 23.5 & 13.5 \\
& & \\
-4 & -6.75 & 16.5 \\
-4 & -4 & 16.5 \\
-4 & 0 & 16.5 \\
-4 & 4 & 16.5 \\
-4 & 6.75 & 16.5 \\
-4 & 13.5 & 16.5 \\
-4 & 23.5 & 16.5
\end{tabular}

\begin{tabular}{lllll} 
& \multicolumn{5}{c}{$\begin{array}{l}\text { required } \\
\text { angles } \\
\text { (radians) }\end{array}$} & \\
$\mathbf{n}$ & Theta 1 & $\begin{array}{l}\text { Theta 2 } \\
1.00\end{array}$ & Theta 3 & Manip \\
0.95 & -2.6301 & 0.4056 & 1.3102 & 7084.4 \\
0.87 & 3.5499 & 1.9044 & 4.8259 & 6754.9 \\
0.80 & 3.3766 & 1.9576 & 4.6635 & 6144.6 \\
0.76 & 3.1783 & 1.8014 & 4.6891 & 5673.5 \\
0.76 & 3.0326 & 1.998 & 4.5107 & 5367.5 \\
0.95 & 2.6809 & 1.9972 & 4.513 & 5379 \\
& 2.2967 & 1.9114 & 4.8069 & 6697.7 \\
0.95 & & & & \\
0.93 & -2.6301 & 0.3921 & 1.1678 & 6740.7 \\
0.86 & 3.5499 & 1.7283 & 4.9618 & 6584.4 \\
0.80 & 3.3766 & 1.7764 & 4.7974 & 6126 \\
0.77 & 3.1783 & 1.8014 & 4.6891 & 5673.5 \\
0.77 & 3.0326 & 1.8094 & 4.6459 & 5462.6 \\
0.92 & 2.6809 & 1.809 & 4.648 & 5472.6 \\
& 2.2967 & 1.7345 & 4.9434 & 6549
\end{tabular}


Appendix A (Continued)

Raptor data from the inverse kinematics program

\begin{tabular}{|c|c|c|c|c|c|c|c|}
\hline & $\begin{array}{l}\text { desired } \\
\text { position }\end{array}$ & & & & $\begin{array}{l}\text { required } \\
\text { angles } \\
\text { (radians) }\end{array}$ & & \\
\hline $\mathbf{x}$ & $\mathbf{y}$ & z & $\mathbf{n}$ & Theta 1 & Theta 2 & Theta 3 & Manip \\
\hline 27.54 & 0 & -29.8 & 0.46 & -1.693 & 2.5314 & 0.4655 & 4173.7 \\
\hline 27.54 & -13.5 & -29.8 & 0.93 & -1.4469 & 1.5685 & 0.2696 & 8474.1 \\
\hline 27.54 & -23.5 & -29.8 & 0.70 & -1.5647 & 0.9437 & 0.3764 & 6387.8 \\
\hline 27.54 & 0 & -22.8 & 0.58 & -1.4343 & 2.4484 & 0.3109 & 5283.4 \\
\hline 27.54 & -13.5 & -22.8 & 0.98 & -1.2072 & 1.5686 & 0.1236 & 8982.7 \\
\hline 27.54 & -23.5 & -22.8 & 0.79 & -1.3199 & 0.9759 & 0.2267 & 7172.4 \\
\hline 27.54 & 0 & -13.8 & 0.60 & -1.1549 & 2.434 & 0.2703 & 5506.1 \\
\hline 27.54 & -13.5 & -13.8 & 0.99 & -0.9308 & 1.5686 & 0.0846 & 9056.1 \\
\hline 27.54 & -23.5 & -13.8 & 0.80 & -1.0427 & 0.9813 & 0.1871 & 7319.6 \\
\hline 27.54 & 0 & -5.82 & 0.53 & -0.9725 & 2.4783 & 0.3781 & 4850.6 \\
\hline 27.54 & -13.5 & -5.82 & 0.97 & -0.7387 & 1.5686 & 0.1877 & 8803.6 \\
\hline 27.54 & -23.5 & -5.82 & 0.76 & -0.8534 & 0.9638 & 0.2923 & 6889.7 \\
\hline 27.54 & 0 & -0.8 & 0.40 & -0.9136 & 2.5776 & 0.5226 & 3661 \\
\hline 27.54 & -13.5 & -0.8 & 0.90 & -0.6564 & 1.5685 & 0.3222 & 8206.9 \\
\hline 27.54 & -23.5 & -0.8 & 0.66 & -0.7775 & 0.9257 & 0.4314 & 6013.9 \\
\hline 27.54 & 0 & 6.18 & 0.00 & & & & 0 \\
\hline 27.54 & -13.5 & 6.18 & 0.66 & -0.6097 & 1.5681 & 0.6209 & 6034.5 \\
\hline 27.54 & -23.5 & 6.18 & 0.35 & -0.7659 & 0.7255 & 0.7523 & 3226.4 \\
\hline 27.54 & 0 & 18.2 & 0.00 & & & & 0 \\
\hline 27.54 & -13.5 & 18.2 & 0.24 & -0.4663 & 1.5664 & 1.0574 & 2200.5 \\
\hline 27.54 & -23.5 & 18.2 & 0.00 & & & & 0 \\
\hline 27.54 & 0 & 24.18 & 0.00 & & & & 0 \\
\hline 27.54 & -13.5 & 24.18 & 0.00 & & & & 0 \\
\hline 27.54 & -23.5 & 24.18 & 0.00 & & & & 0 \\
\hline
\end{tabular}


Appendix A (Continued)

Raptor data from the inverse kinematics program

\begin{tabular}{|c|c|c|c|c|c|c|c|}
\hline & $\begin{array}{l}\text { desired } \\
\text { position }\end{array}$ & & & & $\begin{array}{l}\text { required } \\
\text { angles } \\
\text { (radians) }\end{array}$ & & \\
\hline $\mathbf{x}$ & $y$ & $z$ & $\mathbf{n}$ & Theta 1 & Theta 2 & Theta 3 & Manip \\
\hline 14.04 & 0 & -29.8 & 0.59 & -1.68 & 2.4414 & -0.2922 & 5389.7 \\
\hline 14.04 & -13.5 & -29.8 & 0.78 & -1.4375 & 1.5683 & -0.49 & 7100.6 \\
\hline 14.04 & -23.5 & -29.8 & 0.70 & -1.5617 & 0.9426 & -0.3781 & 6374.5 \\
\hline 14.04 & 0 & -22.8 & 0.48 & -1.4164 & 2.5177 & -0.4457 & 4338.6 \\
\hline 14.04 & -13.5 & -22.8 & 0.62 & -1.1408 & 1.568 & -0.6607 & 5686.7 \\
\hline 14.04 & -23.5 & -22.8 & 0.57 & -1.2803 & 0.8817 & -0.5376 & 5193.9 \\
\hline 14.04 & 0 & -13.8 & 0.44 & -0.997 & 2.5492 & -0.4892 & 3968.5 \\
\hline 14.04 & -13.5 & -13.8 & 0.57 & -0.7071 & 1.5679 & -0.7108 & 5238.8 \\
\hline 14.04 & -23.5 & -13.8 & 0.53 & -0.8531 & 0.8573 & -0.5834 & 4803.7 \\
\hline 14.04 & 0 & -5.82 & 0.53 & -0.6206 & 2.4775 & -0.3766 & 4861.1 \\
\hline 14.04 & -13.5 & -5.82 & 0.70 & -0.3628 & 1.5682 & -0.5827 & 6358.9 \\
\hline 14.04 & -23.5 & -5.82 & 0.63 & -0.4943 & 0.9132 & -0.4654 & 5765.2 \\
\hline 14.04 & 0 & -0.8 & 0.62 & -0.4458 & 2.4248 & -0.2401 & 5653.3 \\
\hline 14.04 & -13.5 & -0.8 & 0.82 & -0.2107 & 1.5684 & -0.4338 & 7509.8 \\
\hline 14.04 & -23.5 & -0.8 & 0.73 & -0.3313 & 0.9566 & -0.3244 & 6696.7 \\
\hline 14.04 & 0 & 6.18 & 0.68 & -0.3118 & 2.3929 & 0.0273 & 6204 \\
\hline 14.04 & -13.5 & 6.18 & 0.98 & -0.0938 & 1.5686 & -0.1558 & 8901.6 \\
\hline 14.04 & -23.5 & 6.18 & 0.83 & -0.2051 & 0.992 & -0.0537 & 7613.5 \\
\hline 14.04 & 0 & 18.2 & 0.00 & & & & 0 \\
\hline 14.04 & -13.5 & 18.2 & 0.72 & -0.1339 & 1.5682 & 0.5603 & 6544.8 \\
\hline 14.04 & -23.5 & 18.2 & 0.42 & -0.2792 & 0.7875 & 0.6855 & 3873.1 \\
\hline 14.04 & 0 & 24.18 & 0.00 & & & & 0 \\
\hline 14.04 & -13.5 & 24.18 & 0.04 & -0.3892 & 1.5594 & 1.3784 & 333.4 \\
\hline 14.04 & -23.5 & 24.18 & 0.00 & & & & 0 \\
\hline
\end{tabular}


Appendix A (Continued)

Raptor data from the inverse kinematics program

\begin{tabular}{|c|c|c|c|c|c|c|c|}
\hline & $\begin{array}{l}\text { desired } \\
\text { position }\end{array}$ & & & & $\begin{array}{l}\text { required } \\
\text { angles } \\
\text { (radians) }\end{array}$ & & \\
\hline$x$ & $\mathbf{y}$ & z & $\mathrm{n}$ & Theta 1 & Theta 2 & Theta 3 & Manip \\
\hline 6.75 & 0 & -29.8 & 0.36 & -1.9533 & 2.6173 & -0.5625 & 3267.4 \\
\hline 6.75 & -13.5 & -29.8 & 0.49 & -1.6302 & 1.5677 & -0.7979 & 4446.1 \\
\hline 6.75 & -23.5 & -29.8 & 0.45 & -1.7901 & 0.8063 & -0.6616 & 4098 \\
\hline 6.75 & 0 & -22.8 & 0.06 & -1.9416 & 3.0336 & -0.7428 & 533.7 \\
\hline 6.75 & -13.5 & -22.8 & 0.26 & -1.3422 & 1.5666 & -1.0329 & 2394 \\
\hline 6.75 & -23.5 & -22.8 & 0.23 & -1.5736 & 0.5819 & -0.8592 & 2137.8 \\
\hline 6.75 & 0 & -13.8 & 0.00 & & & & \\
\hline 6.75 & -13.5 & -13.8 & 0.19 & -0.7349 & 1.5659 & -1.1144 & 1771.3 \\
\hline 6.75 & -23.5 & -13.8 & 0.16 & -1.0226 & 0.4449 & -0.9208 & 1437.6 \\
\hline 6.75 & 0 & -5.82 & 0.23 & -0.5673 & 2.7569 & -0.6599 & 2136.8 \\
\hline 6.75 & -13.5 & -5.82 & 0.37 & -0.1681 & 1.5672 & -0.92 & 3348 \\
\hline 6.75 & -23.5 & -5.82 & 0.34 & -0.3565 & 0.7095 & -0.767 & 3080.1 \\
\hline 6.75 & 0 & -0.8 & 0.42 & -0.2512 & 2.5614 & -0.5041 & 3833.5 \\
\hline 6.75 & -13.5 & -0.8 & 0.56 & 0.0443 & 1.5679 & -0.7283 & 5080.6 \\
\hline 6.75 & -23.5 & -0.8 & 0.51 & -0.1041 & 0.848 & -0.5993 & 4664.3 \\
\hline 6.75 & 0 & 6.18 & 0.63 & -0.0526 & 2.4194 & -0.2197 & 5743.3 \\
\hline 6.75 & -13.5 & 6.18 & 0.84 & 0.1802 & 1.5684 & -0.412 & 7658.6 \\
\hline 6.75 & -23.5 & 6.18 & 0.75 & 0.0607 & 0.9614 & -0.3035 & 6811 \\
\hline 6.75 & 0 & 18.2 & 0.44 & -0.1134 & 2.5482 & 0.4879 & 3979.6 \\
\hline 6.75 & -13.5 & 18.2 & 0.92 & 0.1367 & 1.5685 & 0.2903 & 8374 \\
\hline 6.75 & -23.5 & 18.2 & 0.68 & 0.0174 & 0.9365 & 0.3982 & 6242.3 \\
\hline 6.75 & 0 & 24.18 & 0.00 & & & & 0 \\
\hline 6.75 & -13.5 & 24.18 & 0.46 & -0.0151 & 1.5676 & 0.8275 & 4176.8 \\
\hline 6.75 & -23.5 & 24.18 & 0.02 & -0.2994 & 0.0568 & 0.9933 & 154.4 \\
\hline
\end{tabular}


Appendix A (Continued)

Raptor data from the inverse kinematics program

\begin{tabular}{|c|c|c|c|c|c|c|c|}
\hline & $\begin{array}{l}\text { desired } \\
\text { position }\end{array}$ & & & & $\begin{array}{l}\text { required } \\
\text { angles } \\
\text { (radians) }\end{array}$ & & \\
\hline $\mathbf{x}$ & $y$ & $z$ & $\mathbf{n}$ & Theta 1 & Theta 2 & Theta 3 & Manip \\
\hline 0.54 & 0 & -29.8 & 0.00 & & & & \\
\hline 0.54 & -13.5 & -29.8 & 0.29 & -1.9646 & 1.5668 & -0.9979 & 2680.2 \\
\hline 0.54 & -23.5 & -29.8 & 0.27 & -3.1725 & -0.6259 & -0.8312 & 2427.6 \\
\hline 0.54 & 0 & -22.8 & 0.00 & & & & 0 \\
\hline 0.54 & -13.5 & -22.8 & 0.00 & & & & 0 \\
\hline 0.54 & -23.5 & -22.8 & 0.13 & -1.8579 & 0.8172 & -2.0035 & 1169.5 \\
\hline 0.54 & 0 & -13.8 & 0.00 & & & & 0 \\
\hline 0.54 & -13.5 & -13.8 & 0.00 & & & & 0 \\
\hline 0.54 & -23.5 & -13.8 & 0.00 & & & & 0 \\
\hline 0.54 & 0 & -5.82 & 0.00 & & & & 0 \\
\hline 0.54 & -13.5 & -5.82 & 0.15 & 0.0356 & 1.5652 & -1.1681 & 1400.9 \\
\hline 0.54 & -23.5 & -5.82 & 0.10 & -0.3169 & 0.3155 & -0.9587 & 934.3 \\
\hline 0.54 & 0 & -0.8 & 0.24 & -0.0759 & 2.7438 & -0.6529 & 2229.4 \\
\hline 0.54 & -13.5 & -0.8 & 0.38 & 0.3155 & 1.5672 & -0.9108 & 3428.4 \\
\hline 0.54 & -23.5 & -0.8 & 0.35 & 0.1297 & 0.718 & -0.7594 & 3156.3 \\
\hline 0.54 & 0 & 6.18 & 0.55 & 0.1844 & 2.4645 & -0.3494 & 5044.8 \\
\hline 0.54 & -13.5 & 6.18 & 0.72 & 0.4366 & 1.5682 & -0.5526 & 6608.3 \\
\hline 0.54 & -23.5 & 6.18 & 0.65 & 0.3077 & 0.9236 & -0.4371 & 5972.8 \\
\hline 0.54 & 0 & 18.2 & 0.55 & 0.1168 & 2.4655 & 0.3515 & 5031.1 \\
\hline 0.54 & -13.5 & 18.2 & 0.97 & 0.3477 & 1.5686 & 0.1625 & 8882.5 \\
\hline 0.54 & -23.5 & 18.2 & 0.77 & 0.2338 & 0.9689 & 0.2665 & 6996.9 \\
\hline 0.54 & 0 & 24.18 & 0.00 & & & & 0 \\
\hline 0.54 & -13.5 & 24.18 & 0.63 & 0.1953 & 1.568 & 0.6584 & 5706.8 \\
\hline 0.54 & -23.5 & 24.18 & 0.31 & 0.0305 & 0.6769 & 0.7944 & 2805.5 \\
\hline
\end{tabular}


Appendix A (Continued)

Raptor data from the inverse kinematics data

\begin{tabular}{lcclllll} 
& $\begin{array}{c}\text { desired } \\
\text { position }\end{array}$ & & & \multicolumn{3}{c}{$\begin{array}{l}\text { required } \\
\text { angles } \\
\text { (radians) }\end{array}$} \\
$\mathbf{x}$ & $\mathbf{y}$ & $\mathbf{z}$ & $\mathbf{n}$ & Theta 1 & Theta 2 & Theta 3 & \multicolumn{1}{l}{ Manip } \\
0 & 6.75 & 0 & 0.00 & & & & 0 \\
0 & 4 & 0 & 0.00 & & & & 0 \\
0 & 0 & 0 & 0.28 & -0.0016 & 2.7054 & -0.6301 & 2515.7 \\
0 & -4 & 0 & 0.38 & 0.2121 & 2.3509 & -0.75 & 3471.3 \\
0 & -6.75 & 0 & 0.40 & 0.2933 & 2.1308 & -0.8131 & 3650.2 \\
0 & -13.5 & 0 & 0.40 & 0.3677 & 1.5674 & -0.8816 & 3688.5 \\
0 & -23.5 & 0 & 0.38 & 0.2091 & 0.7842 & -0.7479 & 3462.5 \\
& & & & & & & \\
& & & & & & & \\
0 & 6.75 & 13.5 & 0.00 & & & & \\
0 & 4 & 13.5 & 0.27 & -0.0454 & 2.8654 & 0.1593 & 2424.5 \\
0 & 0 & 13.5 & 0.68 & 0.2157 & 2.3931 & 0.034 & 6200.1 \\
0 & -4 & 13.5 & 0.85 & 0.3358 & 2.1125 & -0.584 & 7788.9 \\
0 & -6.75 & 13.5 & 0.92 & 0.3861 & 1.9466 & -0.1034 & 8394.4 \\
0 & -13.5 & 13.5 & 0.98 & 0.4335 & 1.5686 & -0.1491 & 8920 \\
0 & -23.5 & 13.5 & 0.84 & 0.3223 & 0.9922 & -0.047 & 7619.8 \\
& & & & & & & \\
0 & 6.75 & 16.5 & 0.00 & & & & 0 \\
0 & 4 & 16.5 & 0.00 & & & & 0 \\
0 & 0 & 16.5 & 0.63 & 0.1731 & 2.4205 & 0.2242 & 5724.3 \\
0 & -4 & 16.5 & 0.84 & 0.2968 & 2.1166 & 0.1304 & 7664.4 \\
0 & -6.75 & 16.5 & 0.92 & 0.3473 & 1.9459 & 0.0853 & 8425.3 \\
0 & -13.5 & 16.5 & 1.00 & 0.3944 & 1.5686 & 0.0399 & 9106.8 \\
0 & -23.5 & 16.5 & 0.82 & 0.2832 & 0.9863 & 0.1419 & 7454.9
\end{tabular}


Appendix A (Continued)

Raptor data from the inverse kinematics data

\begin{tabular}{ccclllll} 
& $\begin{array}{c}\text { desired } \\
\text { position }\end{array}$ & & & & \multicolumn{3}{c}{$\begin{array}{l}\text { angles } \\
\text { (radians) }\end{array}$} \\
$\mathbf{x}$ & $\mathbf{y}$ & $\mathbf{z}$ & $\mathbf{n}$ & Theta 1 & Theta 2 & Theta 3 & Manip \\
-4 & 6.75 & 13.5 & 0.00 & & & & 0 \\
-4 & 4 & 13.5 & 0.28 & 0.1008 & 2.8484 & 0.1243 & 2595.9 \\
-4 & 0 & 13.5 & 0.68 & 0.3569 & 2.3925 & -0.0007 & 6211.4 \\
-4 & -4 & 13.5 & 0.84 & 0.4837 & 2.116 & -0.123 & 7681.5 \\
-4 & -6.75 & 13.5 & 0.91 & 0.5277 & 1.9483 & -0.1383 & 8318 \\
-4 & -13.5 & 13.5 & 0.97 & 0.5754 & 1.5686 & -0.1842 & 8815.1 \\
-4 & -23.5 & 13.5 & 0.83 & 0.4637 & 0.9907 & -0.0817 & 7578.5 \\
& & & & & & & \\
-4 & 6.75 & 16.5 & 0.00 & & & & 0 \\
-4 & 4 & 16.5 & 0.01 & -0.0647 & 3.1311 & 0.3176 & 86.1 \\
-4 & 0 & 16.5 & 0.64 & 0.306 & 2.4121 & 0.1888 & 5865 \\
-4 & -4 & 16.5 & 0.85 & 0.4284 & 2.1142 & 0.0955 & 7736.5 \\
-4 & -6.75 & 16.5 & 0.93 & 0.4787 & 1.945 & 0.0506 & 8468.4 \\
-4 & -13.5 & 16.5 & 1.00 & 0.5257 & 1.5686 & 0.0052 & 9121 \\
-4 & -23.5 & 16.5 & 0.83 & 0.4149 & 0.9892 & 0.1069 & 7534.7
\end{tabular}


Appendix B: Inverse Kinematic Program

This is a list of all the programs that are called out in the description of the inverse kinematics:

\section{robot.m}

function robot( initial_postion,theta1,theta2,theta3,final_position,steps )

$\%$ the robot function is the "main" function of this set of functions

$\%$ input is the initial arm angles $\mathrm{t} 1, \mathrm{t} 2, \mathrm{t} 3$, final endeffector position, $\mathrm{xyz}$, and the number of steps to use

$\%$ set inputs of the initial theta angles in radians

$\% \quad$ theta1=3.7437;

$\%$ theta2 $=2.6441$;

$\% \quad$ theta3 $=4.5093$;

theta1 $=-2.2234$;

theta2 $=-.6703$;

theta3=2.0199;

$\%$ set the number of steps (resolution) to use from initial to final

$\%$ steps = input('how many intermediate steps?'); steps $=15$;

$\%$ set the tolerance at which the algorithm will loop to before moving on to the next waypoint

$\%$ this is a position tolerance $x, y, z$ of the edeffector tolerance $=[0.001 ; 0.001 ; 0.001]$; 
Appendix B (Continued)

$\%$ set manip_thres which is where the move_arm algorithim will break out of the loop if the manipulability becomes too low

$\%$ this is an indicator of if the arm is moving toward an singularity and keeps it from going into an infinite loop

manip_thres $=50$;

$\%$ set the desired final position of the endeffector $[\mathrm{x} ; \mathrm{y} ; \mathrm{z}]$

final_position = input('final position -eg $[5 ; 5 ; 5]=$ ') \%[0;0;0]

$\%$ Build current_state structure, a structure is used to simplify moving variable data between functions

$\%$ set the current state as the initial theta and position

initial_theta $=[$ theta1, theta2, theta3];

initial_position = find_position(initial_theta);

current_state $=$ struct('xyz',\{initial_position\},'angles',\{initial_theta\})

$\%$ calculate array of waypoints which is a set of points on the line that connects the initial position point to the final point

$\%$ returns a three dimension waypoint matrix called the_plan

disp('calculating plan of attack')

the_plan = plot_waypoints(initial_position, final_position, steps);

$\%$ move the arm, check amount of error, continue with new waypoint if error $<$ tolerance

$\%$ initialize error $=0$ to make a "do loop" like loop

$\%$ the points array is an array of points traveled to by the arm 
Appendix B (Continued)

$\%$ the angles array is an array of angles traveled to by the arm

$\%$ set the array to "zeros" for more efficient processing

points $=$ zeros $(3$, steps $)$;

angles = zeros $($ steps, 3$)$;

$\%$ initialize a placeholder variable $\mathrm{n}$ for loop counting

$\% \mathrm{n}$ is used to count the number of times the arm move is attempted

$n=0$;

$\%$ a for loop from 1 to steps +1

for $\mathrm{i}=1$ :(steps +1$)$

fprintf('Calculating a waypoint, iteration number \%iln', i)

next_position = the_plan(:,:,i);

$\%$ reset postion error for next loop

error $=[99 ; 99 ; 99]$;

$\%$ end the for loop if the manipiablilty goes below the manip_thres

if ( manip_thres > (abs(manip(current_state.angles $)))$ )

disp('approaching singularity!');

manip_break=1;

break;

end

$\%$ loop the move_arm function and update the current_state

$\%$ if the error is > than the tolerance move_arm and update current_state again

while any(error > tolerance)

current_state $=$ move_arm(current_state, next_position);

error = next_position - current_state.xyz;

$\%$ current_state.xyz 
Appendix B (Continued)

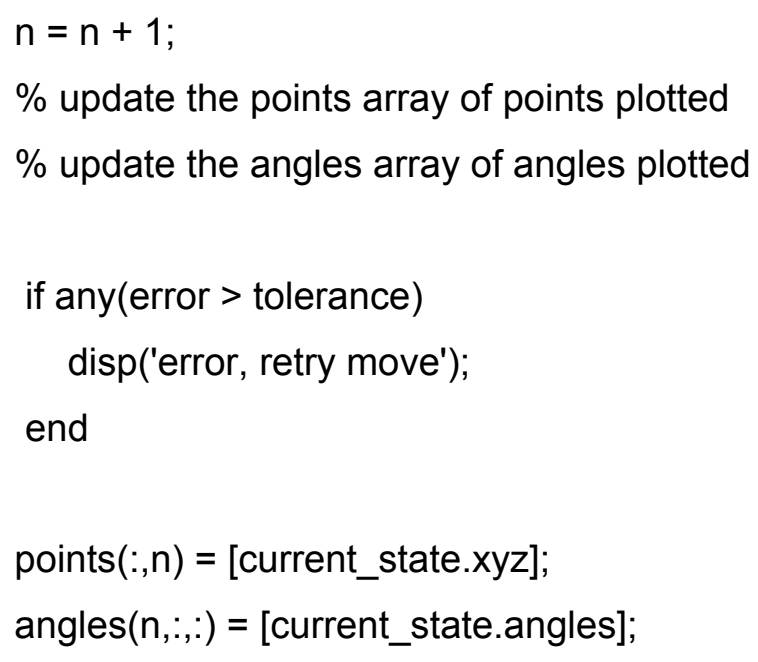

$\%$ keep it from becoming an infinite loop

$\%$ end the for loop if the manipiablilty goes below the manip_thres

if ( manip_thres > (abs(manip(current_state.angles $)))$ )

disp('approaching singularity');

manip_break=1;

break;

end

end $\%$ end of the while loop

end $\%$ end of the for loop

disp('Waypoints; The steps taken to get from intial to final position') disp('Including all iterative substeps (subloop steps)')

$\%$ display the points array

points 
Appendix B (Continued)

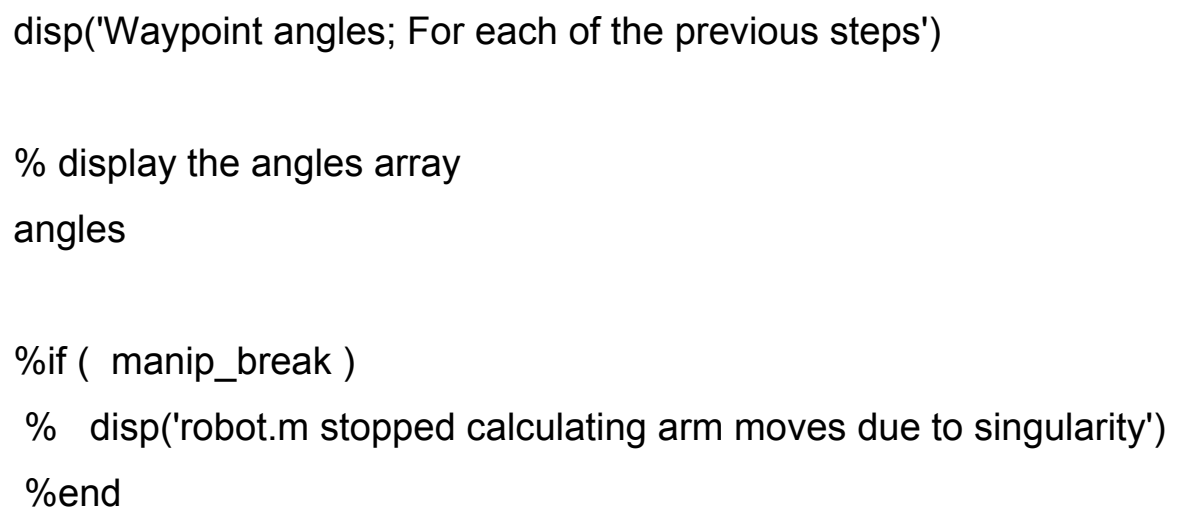

This is the first subroutine called out from robot.m is find_position.m:

\section{find_position.m}

function position = find_position( theta )

$\%$ the find_position function takes input of the arm angles and returns the position of the endeffector

$\%$ set $\mathrm{t} 1, \mathrm{t} 2, \mathrm{t} 3$ to the current state angles from the input of the structure

$$
\begin{aligned}
& \mathrm{t} 1=\text { theta(1); } \\
& \mathrm{t} 2=\text { theta(2); } \\
& \mathrm{t} 3=\text { theta(3); }
\end{aligned}
$$

$\%$ the three position equations specific for each robot arm configuration

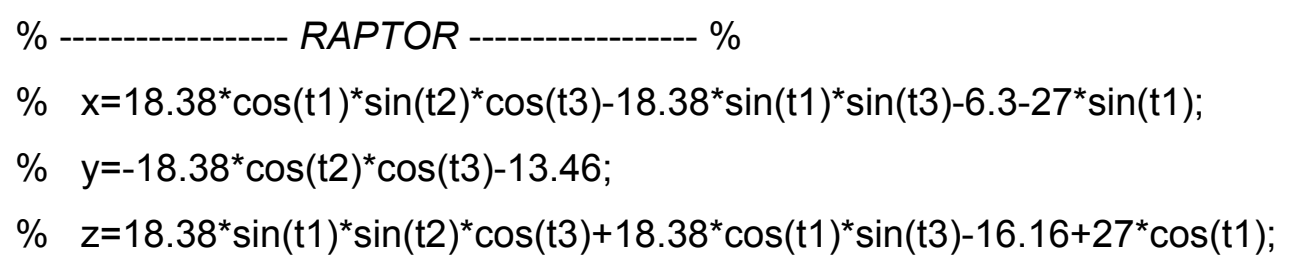


Appendix B (Continued)

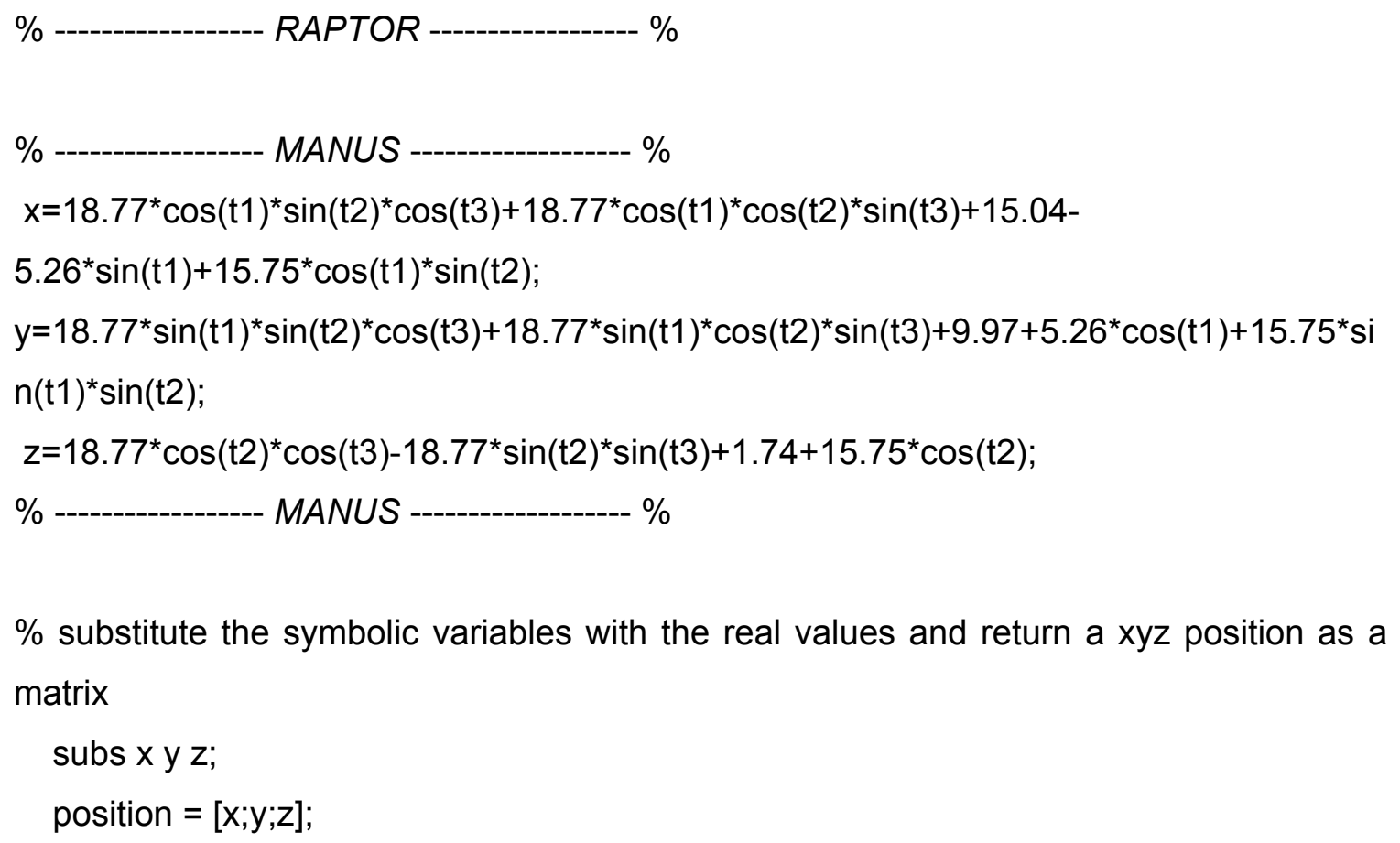

$\%$ substitute the symbolic variables with the real values and return a xyz position as a matrix

subs $x$ y $z$;

position $=[x ; y ; z]$

The second called out subroutine is plot_waypoints.m

\section{plot_waypoints.m}

function plot=plot_waypoints(initial_position, final_position, steps)

$\%$ sub function to determine all desired points in straight line between inital_pos and final desired

\%find out what to increment $x$ vector for 'steps' number of steps increment=(final_position-initial_position)/steps;

\%i know i want to pre allocate some array space to same time $x=$ zeros $(3,1$, steps $)$;

$\%$ plot out points $x(:,:, 1)$ is initial point

$\%$ had to use for beginning with 1 due to language constraint 
Appendix B (Continued)

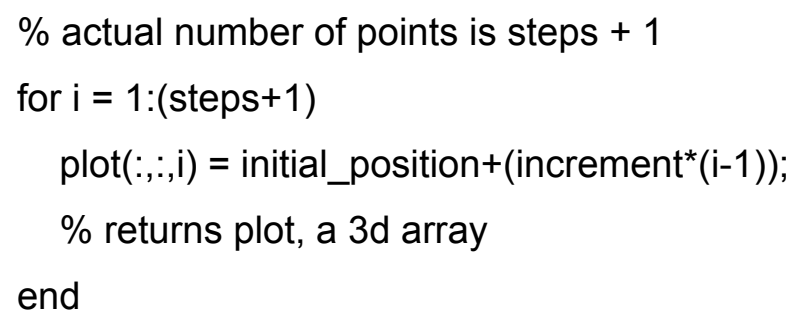

$\%$ the three position equations specific for each robot arm configuration

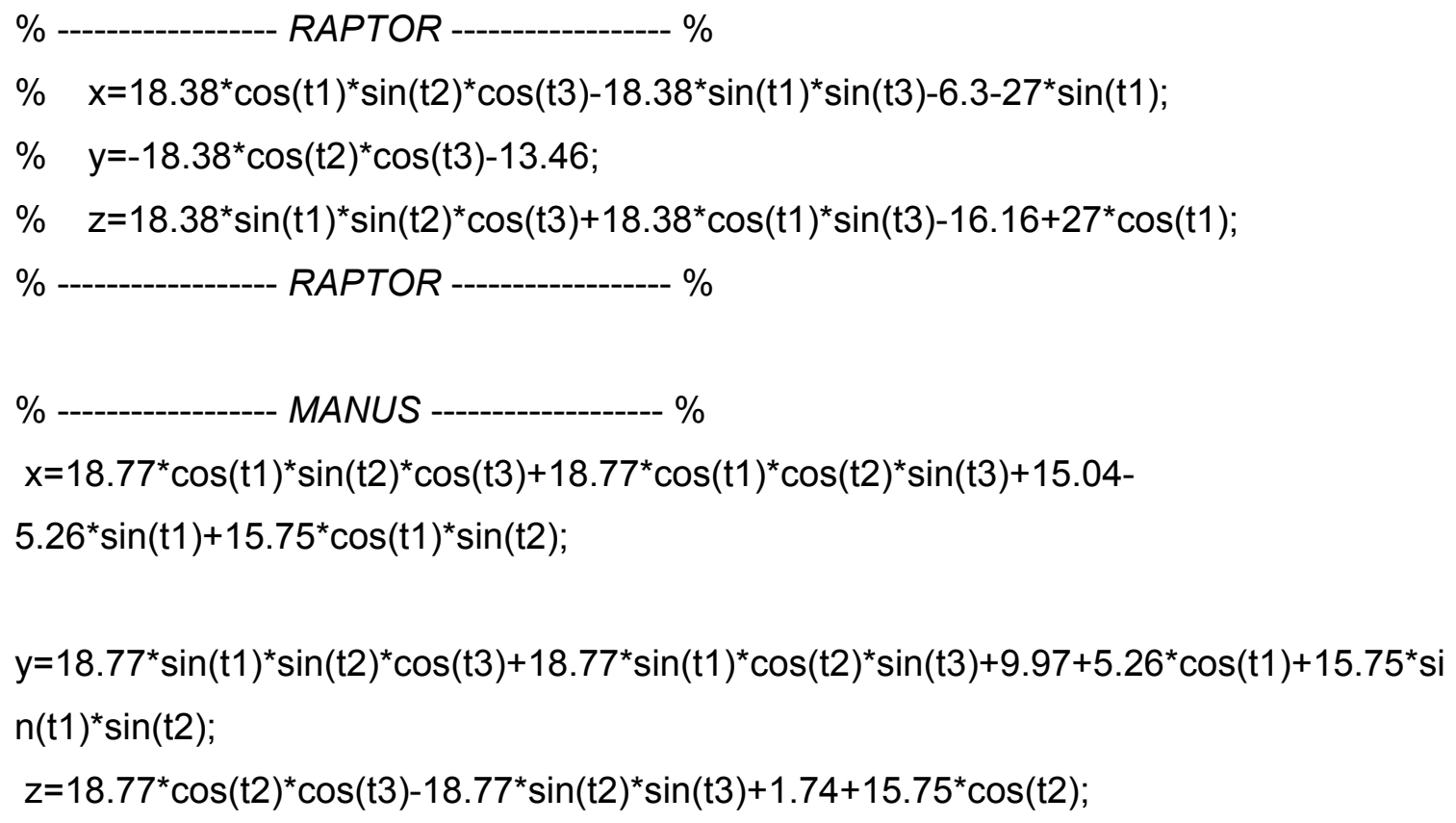


Appendix B (Continued)

\% -------------- MANUS ----------------- \%

$\%$ calculate the jacobian matrix of the position equation

$\mathrm{J}=$ jacobian $([\mathrm{x} ; \mathrm{y} ; \mathrm{z}],[\mathrm{t} 1 \mathrm{t} 2 \mathrm{t} 3])$;

$\%$ calculate the determinate of the jacobian matrix

$\mathrm{J}=\operatorname{det}(\mathrm{J})$

$\%$ prepare to calculate a real number

$\%$ set the symbolic variables to real numbers, the angles of the arm

$\mathrm{t} 1=$ theta $(1)$

$\mathrm{t} 2=$ theta $(2)$;

t3 = theta $(3)$;

$\%$ substitute the $\mathrm{t} 1, \mathrm{t} 2, \mathrm{t} 3$ in the equation $\mathrm{J}$ and return $\mathrm{m}$

$\mathrm{m}=\operatorname{subs}(\mathrm{J})$;

The fourth called out program is move_arm.m

move_arm.m

function new_state $=$ move_arm(current_state, next_position)

$\%$ The move_arm function takes input of the current_state structure and the next_position variable

$\%$ returns the new position of the end effector and the arm angles

$\%$ calc increment between the current position and the next position increment $=$ next_position - current_state.xyz;

$\%$ calc new theta given the current state angles and the incremental position new_theta $=$ get_theta(current_state.angles, increment);

$\%$ calc the new position given the newly calculated theta new_position = find_position(new_theta); 
Appendix B (Continued)

$\%$ return the new position and new thetas via a structure new_state $=\operatorname{struct}(' x y z ',\{$ new_position $\}, ' a n g l e s ',\{$ new_theta $\})$;

The fifth and final called out subroutine is get theta.m

\section{get_theta.m}

function new_theta=get_theta(theta, increment)

$\%$ the get_theta function takes input of the robot arm angles and the position increment $\%$ outputs a new set of angles which is used to approximate the new position

$\%$ the jacobian matrix and its inverse are calculated symbolically syms $\mathrm{t} 1 \mathrm{t} 2 \mathrm{t} 3$

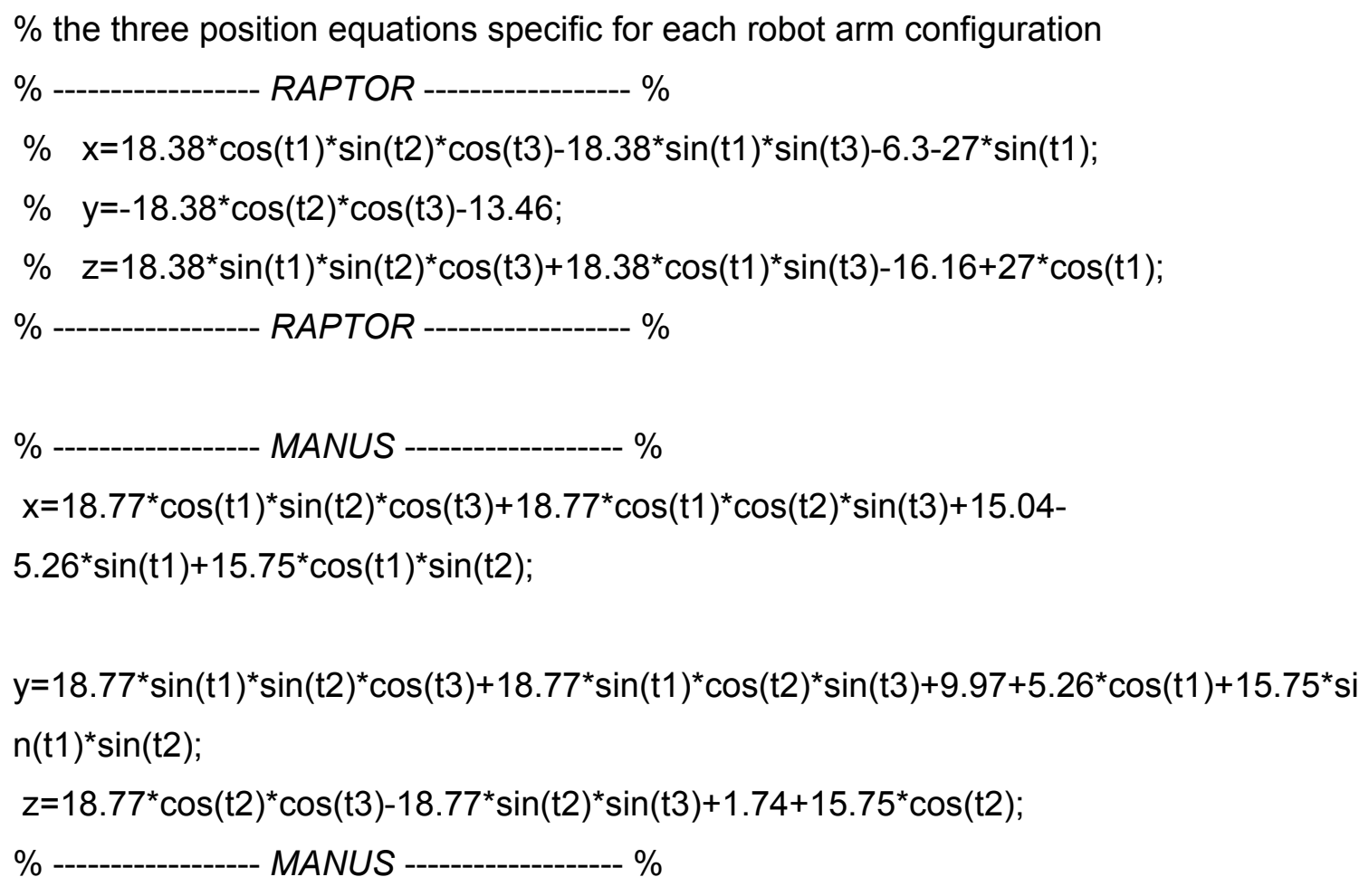

$\%$ calculating the jacobian 
Appendix B (Continued)

$$
\begin{aligned}
& \mathrm{J}=\text { jacobian }([\mathrm{x} ; \mathrm{y} ; \mathrm{z}],[\mathrm{t} 1 \mathrm{t} 2 \mathrm{t} 3]) ; \\
& \text { \% calculating the inverse jacobian } \\
& \text { inverse_jacobian }=\operatorname{inv}(\mathrm{J}) ; \\
& \% \text { calculating the delta theta } \\
& \text { delta_theta=inverse_jacobian*increment; }
\end{aligned}
$$

$\%$ substituting the current angles into the delta_theta equation

$$
\begin{aligned}
& \mathrm{t} 1=\text { theta(1); } \\
& \mathrm{t} 2=\text { theta(2); } \\
& \mathrm{t} 3=\text { theta(3); }
\end{aligned}
$$

$\%$ calculating delta_theta

delta_theta = subs(delta_theta);

$\%$ calculate the new theta or the intermediate theta

$\%$ delta thetas are summed with the current thetas

$\%$ disp('Waypoint 1 Theta = Original theta + Delta theta')

new_theta $=[\mathrm{t} 1+$ delta_theta $(1,1) ; \mathrm{t} 2+$ delta_theta $(2,1) ; \mathrm{t} 3+$ delta_theta(3,1)]; 
